\title{
Diffusive Mesh Relaxation in ALE Finite Element Numerical Simulations
}

\author{
RECEIVI: \\ Eveline I. Dube \\ AUG 169990 \\ (Ph.D Thesis) \\ OSTI
}

June 1996

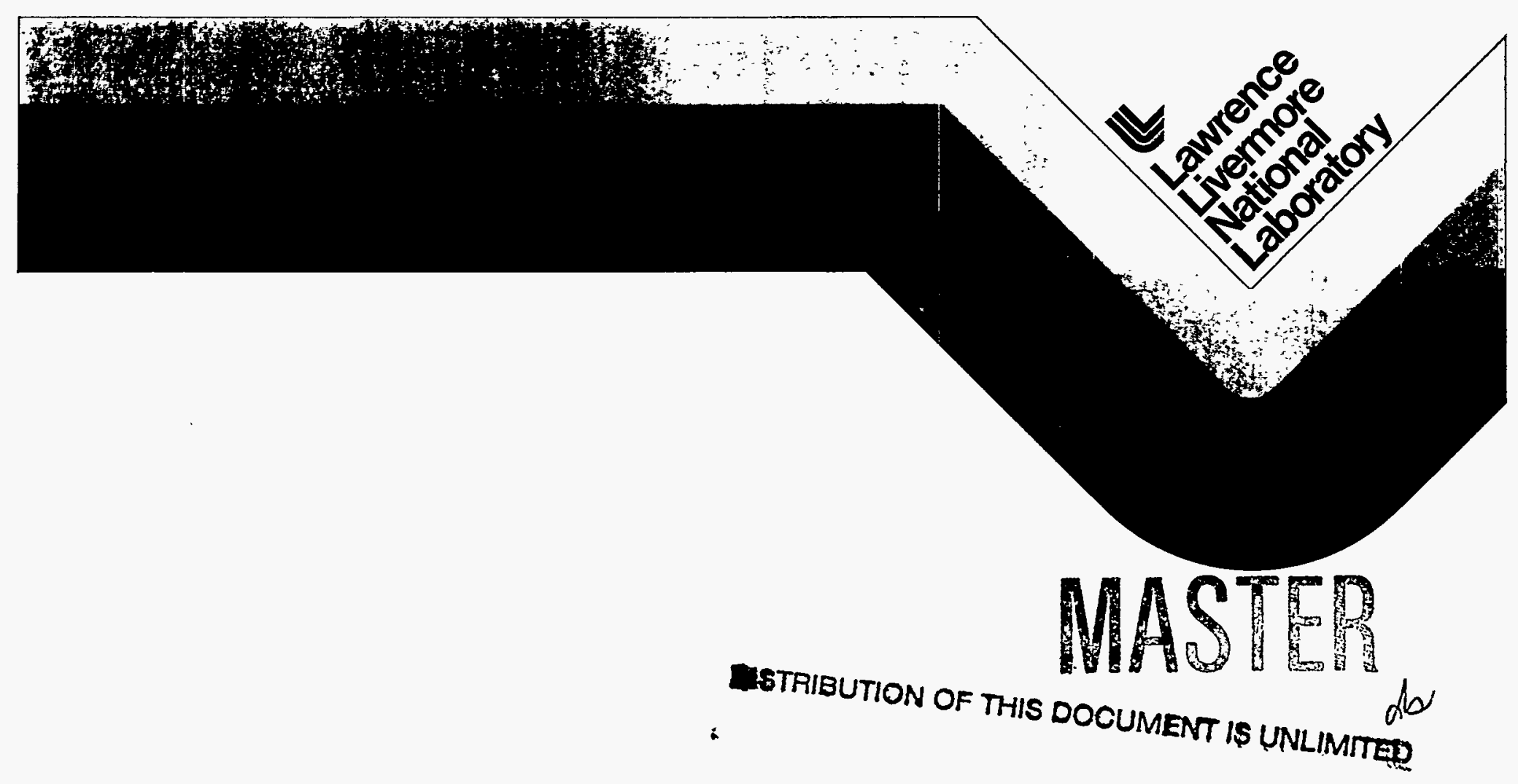




\section{DISCLAIMER}

This document was prepared as an account of work sponsored by an agency of the United States Government. Neither the United States Government nor the University of California nor any of their employees, makes any warranty, express or implied, or assumes any legal liability or responsibility for the accuracy, completeness, or usefulness of any information, apparatus, product, or process disclosed, or represents that its use would notinfringe privately owned rights. Reference herein to any specific commercial product, process, or service by trade name, trademark, manufacturer, or otherwise, does not necessarily constitute or imply its endorsement, recommendation, or favoring by the United States Government or the University of California. The views and opinions of authors expressed herein do not necessarily state or reflect those of the United States Government or the University of California, and shall not be used for advertising or product endorsement purposes.

This report has been reproduced directly from the best available copjr.

Available to DOE and DOE contractors from the Office of Scientific and Technical Information P.O. Box 62, Oak Ridge, TN 37831

Prices available from (615) 576-8401, FTS 626-8401

Available to the public from the National Technical Information Service

U.S. Department of Commerce 5285 Port Royal Rd., Springfield, VA 22161

Workperformed under the auspices of the U.S. Department of Energy by Lawrence Livermore National Laboratory under Contract W-7405-Eng-48. 


\title{
Diffusive Mesh Relaxation in ALE Finite Element Numerical Simulations
}

\author{
Eveline I. Dube
}

(Ph.D Thesis)

Manuscript date: June 1996

LAWRENCE LIVERMORE NATIONAL LABORATORY University of California - Livermore, California • 94551 


\section{DISCLAIMER}

Portions of this document may be illegible in electronic image products. Images are produced from the best available original document. 


\section{Diffusive Mesh Relaxation in ALE Finite Element Numerical Simulations}

by

EVELINE INGEBORG DUBE

B.S. (University of Arizona) 1983

M.S. (University of California, Davis) 1989

\section{DISSERTATION}

Submitted in partial satisfaction of the requirements for the degree of

DOCTOR OF PHILOSOPHY

in

Engineering-Applied Science

of the

\section{UNIVERSITY OF CALIFORNIA}

\section{DAVIS}

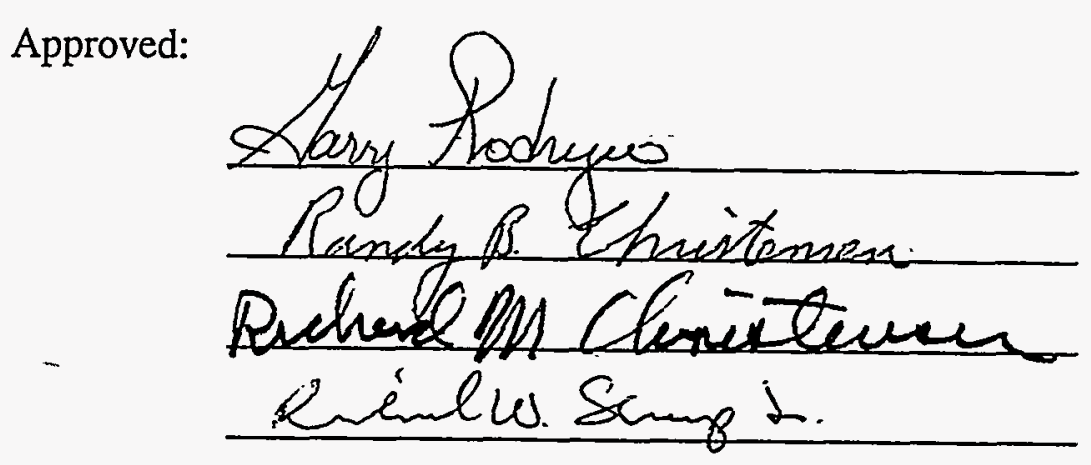

Committee in Charge 
Abstract 4

List Of Symbols

Acknowledgments 6

I. Introduction 7

$\begin{array}{ll}\text { Motivation } & 7\end{array}$

$\begin{array}{ll}\text { Organization } & 9\end{array}$

II. Grid Generation 11

Elliptic Partial Differential Equations $\quad$ _ 11

Elliptic Grid Generators $\quad 13$

Calculus of Variations $\quad 21$

Variational Grid Generation $\quad 24$

Summary 29

III. Diffusive Mesh Relaxation 30

The Problem 30

Motivation $\quad 30$

Variational Formulization $\quad 35$

Galerkin Finite Element Formulization $\quad 37$

Coordinate Transformation 39

Final Application of Galerkin Finite Element Approach $\quad 40$

Determination of the Geometric Weight ${ }^{-} \quad 54$

IV. Hourglass Control $\quad 56$

Motivation 56

Definition of Hourglassing 58

A Simple Two-Dimensional Example $\quad 60$

Hourglass Control in the Diffusive Mesh Relaxer 73

V. Lagrangian Mechanics 93

Introduction 93

Continuum Mechanics 93

Lagrangian Frame of Reference 96

$\begin{array}{ll}\text { VI. Application and Results } & 100\end{array}$ 
$\begin{array}{ll}\text { Preliminary 2D Structured Mesh Findings } & 100\end{array}$

$\begin{array}{ll}\text { ALE3D } & 104\end{array}$

Test Problem: A Cube 105

Test Problem: Spherical Symmetry 116

Test Problem: Air Flow Through a Diverging Nozzle 121

Test Problem: Spherical Symmetry With a Push at One Point 124

Test Problem: Aerostructural Test Case 132

VII. Conclusions $\quad 136$

$\begin{array}{ll}\text { VIII. References } & 138\end{array}$ 
Eveline Ingeborg Dube

June 1996

Engineering-Applied Science

\title{
Diffusive Mesh Relaxation in ALE Finite Element Numerical Simulations
}

\begin{abstract}
The theory for a diffusive mesh relaxation algorithm is developed for use in threedimensional Arbitrary Lagrange/Eulerian (ALE) finite element simulation techniques. This mesh relaxer is derived by a variational principle for an unstructured 3D grid using finite elements, and incorporates hourglass controls in the numerical implementation. The diffusive coefficients are based on the geometric properties of the existing mesh, and are chosen so as to allow for a smooth grid that retains the general shape of the original mesh. The diffusive mesh relaxation algorithm is then applied to an ALE code system, and results from several test cases are discussed.
\end{abstract}




\section{List Of Symbols}

हे

vector $\varepsilon$

B

matrix B

J . Jacobian matrix

$j \quad$ the jacobian, or the determinant of $\mathbf{J}, \operatorname{det}(\mathbf{J})$

$N_{i} \quad$ shape, or basis function for ith node

$R^{n} \quad$ n-dimensional Euclidean space

$u_{x} \quad$ partial derivative of $u$ with respect to $x, \frac{\partial u}{\partial x}$

$N_{1, \xi} \quad$ partial derivative of $N_{1}$ with respect to $\xi, \frac{\partial N_{1}}{\partial \xi}$

$d x^{3} \quad d x d y d z$ 


\section{Acknowledgments}

To succeed in an endeavor such as completing a dissertation requires the love and encouragement of many people. I wish to thank my advisor, Dr. Garry Rodrigue, for coming up with the computational program under the applied science field, and for hav-* ing the patience to handle the unpredictable workload of a full-time employee. I would never have been able to accomplish this without the support of Dr. Richard Sharp and Linnea Cook, who allowed me the time for many years to pursue my goal. To all my work walking friends, Nancy Alexander, Joanne Norman, Pat Cole, and Lauri Dobbs, I appreciate the walks and talks, without which I would have gone insane! To Dr. Judy Kammeraad, Dr. Cynthia Nitta, Jeff Long, and Al and Lila Leibee, your warmth and friendship gave me hope that this would someday end. I also wish to thank my coworkers in B Group and ALE3D for putting up with me, and the management in Computations and B Division for agreeing to this long process.

To my dissertation committee members, Dr. Richard Christensen and Dr. Randy Christensen, I give a special thanks. The knowledge I gained from them will last a lifetime.

-Finally, I wish to thank my family. Anyone lucky enough to be surrounded with the love and support that I have is sure to succeed. I would never have had the courage to undertake this goal without the love and support of all of them. Mom and Dad, you gave me the world. Pete, you gave me strength. Brian, you gave me encouragement. Michael, you gave me love. Thank You. 


\section{Chapter 1: Introduction}

\section{Motivation}

Continuum problems in engineering and physics, such as calculations of metal forming operations including extrusion, casting and rolling, are traditionally solved in one of two techniques. With the Lagrangian technique, the numerical mesh flows with the mass. One significant positive attribute of this method is the ability to apply variable zoning within the continuum, allowing the user the option of higher resolution in regions of interest. The problem with this approach is the possibility of crossed zones within the mesh hindering the calculation. In the Eulerian method, the mass moves through the numerical mesh. Although this approach eliminates the possibilities of crossed zones, the inability to have variable zoning requires the finest zoning to be applied throughout the problem, greatly decreasing the speed, and increasing the memory requirements for a given calculation.

Over the past 15 years, $\mathrm{ALE}^{7,8,17,36}$ (arbitrary Lagrangian-Eulerian) calculations have been developed in hopes of combining the best attributes of the Lagrangian and Eularian techniques. An ALE formulation consists of two steps: a complete Lagrangian calculation followed by a modified Eulerian, or advection step. This advection step also consists of two parts. First, the mesh is regenerated, or relaxed, to alleviate any tangled or irregular zones created by the Lagrangian calculation.This new mesh needs to be smooth, with no bow-ties or distorted elements, and no drastic changes in zone size. Next, the zonal quantities are remapped from the old mesh to the new relaxed mesh using various conservative convection schemes. ${ }^{41}$

This dissertation focuses on the first part of the advection step, the mesh relaxation 
phase. This process involves taking an existing mesh, and generating a new, smooth mesh. Grid generation schemes such as those described in Chapter 2 begin with only a knowledge of the boundary of the physical region. These schemes then proceed to generate a grid on the physical region that satisfactorily represents the geometry of the region. In mesh relaxation, a grid exists on the physical region that is unsatisfactory for some reason, for example the grid does not allow for accurate numerical simulations to be computed on it. Although this grid may represent the geometry of the physical region adequately, it needs to be readjusted. Mesh relaxation, then, can be regarded as a grid generation problem whereby the new grid is generated using information from the initial, although unsatisfactory grid.

Mesh relaxation, or smoothing, of an existing structured mesh has been done in some manner over the past 20 years, within several contexts. For example, many mesh generators ${ }^{25,32}$ include a smoothing operation on a newly generated mesh to reduce irregularites and produce a more regular grid. As another example, some Lagrangian . calculations consist of service packages which let the user service, or fix, a tangled mesh during a calculation using a simple Laplacian smoothing technique. Current research on methods for unstructured meshes consisting of Delaunay Triangulations has been published, where it has been found that Laplacian smoothing techniques can greatly increase the regularity of the mesh and improve the triangular shapes. ${ }^{10,12}$. Smoothing unstructured, hexahedral elements, however, is a relatively new field.

An additional requirement for the relaxation algorithm is that it maintain the existing zone structure of the initial mesh. In other words, finely zoned regions need to remain finely zoned, but not crossed, and coarsely zoned regions must remain coarse. This 
requirement is important when a simulation problem has fined zoned regions in areas of fast developing dynamics, for example. If the mesh relaxation algorithm destroys this zoning structure, the accuracy of the numerical simulation will be questionable.

This dissertation derives an unstructured, hexahedral mesh relaxation approach for use in ALE simulations. To be successful, this method must produce smooth, regular meshes, and have the ability to maintain initial zoning structures. The diffusive mesh relaxation technique for ALE simulations developed in this dissertation succeeds, as will be seen in the five test cases discussed in Chapter 6.

\section{Organization}

This paper begins with an overview of mesh generation. The techniques used for generating an initial grid are obvious choices for mesh relaxation, and the positive and negative attributes of the individual mesh generation algorithms will similarly affect relaxation approaches. Next, the diffusive mesh relaxation technique is derived, followed by a discussion on hourglass control. Hourglassing is an nonphysical phenomena found in Lagrangian calculations, which, if not controlled, will produce unstable, irregular meshes.

As a lead into the applications section, a short discussion on Lagrangian mechanics is provided. In the applications and results section, preliminary $2 \mathrm{D}$ structured mesh findings are presented, followed by a brief section on ALE3D, an ALE simulation program in which the diffusive mesh relaxation algorithm is applied. Five test problems are discussed and the results of the diffusive mesh relaxer are compared with those of a default, existing mesh relaxation algorithm. In two test cases, the diffusive mesh relaxation technique derived in this dissertation was a necessity for the simulation to run, and in another 
test problem, the resulting mesh from this algorithm was better than that of the default relaxer.

This paper concludes with a summary. 


\section{Chapter 2: Grid Generation}

Numerical grid generation is an important component in computationally solving scientific problems. For example, when a continuum mechanics problem is to be solved numerically, the continuum needs to be converted to a finite set of grid points. For problems with complex shaped regions, this set of grid points needs to be adapted to the shape of the region, giving a boundary-fitted or boundary-conforming grid.

There are several approaches taken in generating the grid. One of these techniques is the interpolation or algebraic method ${ }^{35}$. Algebraic techniques involve the use of an interpolating function which is constrained to match specified boundary values. Directly solving the function, then, defines the new interior mesh points. Another more popular approach is to generate a grid using elliptic partial differential equations. This latter approach is the one discussed in the remainder of this section.

The first section of this chapter discusses several properties of elliptic partial differential equations which lend themselves well to mesh generation. Based on these properties, a discussion of various elliptic generators will follow. The first set of elliptic mesh generators to be presented are solved using a direct approach. Next, since the variational approach to grid generation has proven successful in controlling mesh points, it is useful to discuss the concept of Calculus of Variations, and then present several variational mesh generator algorithms.

\section{Elliptic Partial Differential Equations}

Consider the linear partial differential equation ${ }^{27}$

$$
P(u)=A(u)+a u=f
$$


where

$$
A(u)=\sum_{i, k=1}^{n} a_{i k}(x) u_{x_{i} x_{k}}+\sum_{i=1}^{n} a_{i}(x) u_{x_{i}} \text {, }
$$

$u_{x_{i} x_{k}}=\frac{\partial^{2} u}{\partial x_{i} \partial x_{k}}$ and $u_{x_{i}}=\frac{\partial u}{\partial x_{i}}$. This equation is valid on the bounded domain $\Omega \subset R^{n}$ with $\partial \Omega$, the boundary of $\Omega$, being sufficiently smooth, and the functions $a_{i k}, a_{i}, a$, and $f$ are all continuous in $\Omega$ with $a_{i k}(x)=a_{k i}(x), x \in \Omega, i, k=1,2, \ldots, n . P$ is defined as an elliptic partial differential equation if for any $x \in \Omega$, and any $\xi=\left(\xi_{1}, \xi_{2}, \ldots, \xi_{n}\right) \neq 0$, the following inequality holds:

$$
\sum_{i, k=1}^{n} a_{i k}(x) \xi_{i} \xi_{k}>0
$$

Elliptic equations are typically associated with equilibrium or steady-state problems. The solutions of these partial differential equations represent steady-state solutions, which satisfy the PDE at every point inside the bounded domain $\Omega \subset R^{n}$ and satisfy certain conditions at every point on $\partial \Omega$.

Solutions to elliptic PDEs obey three basic principles ${ }^{27}$. The first principle states that the solutions are smooth provided their coefficients are also smooth. In other words, the solutions and their coefficients are continuous and have continuous derivatives. Second, the solutions are determined by their values on the boundary. Thirdly, the solutions to elliptic equations obey an extremum principle, or in other words, the values of all points inside and on the boundary of $\Omega$ are bounded by the values on this boundary, $\partial \Omega$. The 
following theorem states the strong extremum principle for solutions of elliptic partial differential equations defined by Eq. 1 .

Theorem: Suppose that $a(x) \leq 0$ in $\bar{\Omega}$. If $f(x) \leq 0$ or $f(x) \geq 0$

in $\bar{\Omega}$, then every nonconstant solution of Eq. 1 attains its negative minimum, or positive maximum on $\partial \Omega$, and not in $\Omega$.

The extremum property, along with the smoothness found in solutions to elliptic systems, are two of the advantages in using a system of elliptic partial differential equations as a means of mesh generation. These advantages allow grids, produced by various elliptic partial differential equations, to be generated for many configurations without an overlap of grid lines ${ }^{34}$. The desired characteristic of non-overlapping grid lines is further discussed in the next section. A disadvantage in using elliptic partial differential equations is that this full system of equations must be solved to generate the mesh coordinates. The advantages, however, far outweighed the disadvantages, and the use of elliptic grid generators, based on these principles, has proven vital to computing solutions to partial differential equations in fluid dynamics and continuum mechanics

The following section gives a somewhat historical outline of elliptic grid generation, along with the primary advantages and disadvantages of the various algorithms. But first, the numerical solution basics such as logical and physical spaces, and coordinate transformations are provided.

\section{Elliptic Grid Generators}

Grid generation arose from the need to compute solutions to partial differential equations for fluid dynamics and continuum mechanics problems, some with physical 
regions of complex shape. Due to the difficulty of solving these equations on complex regions, the physical region is transformed ${ }^{19}$, or mapped, to a simpler shape, sometimes called the logical space. The advantage of this transformation is that the complexity of the region is removed and the boundary conditions can be more easily approximated. The disadvantage is that the partial differential equations need also be transformed to this logical space, and are usually much more complex to solve.The transformation can be thought of as a general curvilinear coordinate system for the physical space, or as a mapping between the logical and physical space.

It is possible, then, to transform this physical region into a square for two dimensions, as shown in Fig. 1 , or a cube for three dimensions so that the boundary of the logical region corresponds to the boundary of the physical region, giving rise to a boundary conforming coordinate system. Because grids are first chosen in the logical space and then mapped to the physical space, it is natural to view the transformation as mapping from logical to physical space.

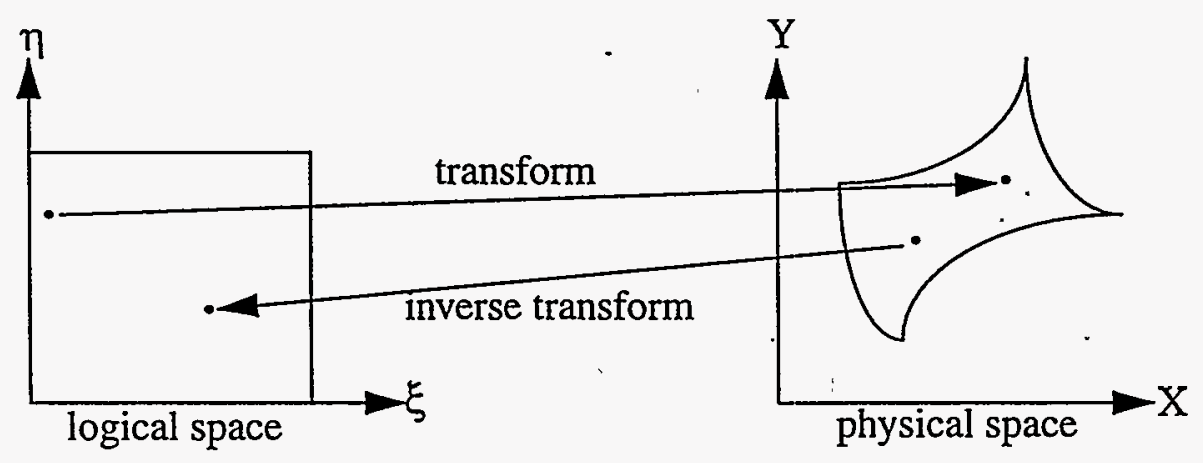

Fig. 1: Transformation, or map, of coordinate system.

If a set of points is chosen in the logical region, then the transformation maps these points to points in the physical space. Furthermore, if the points in the logical region are arranged as rectangles, then the resulting grid in the physical region will be rectangular 
in structure, as seen in Fig. 2 .
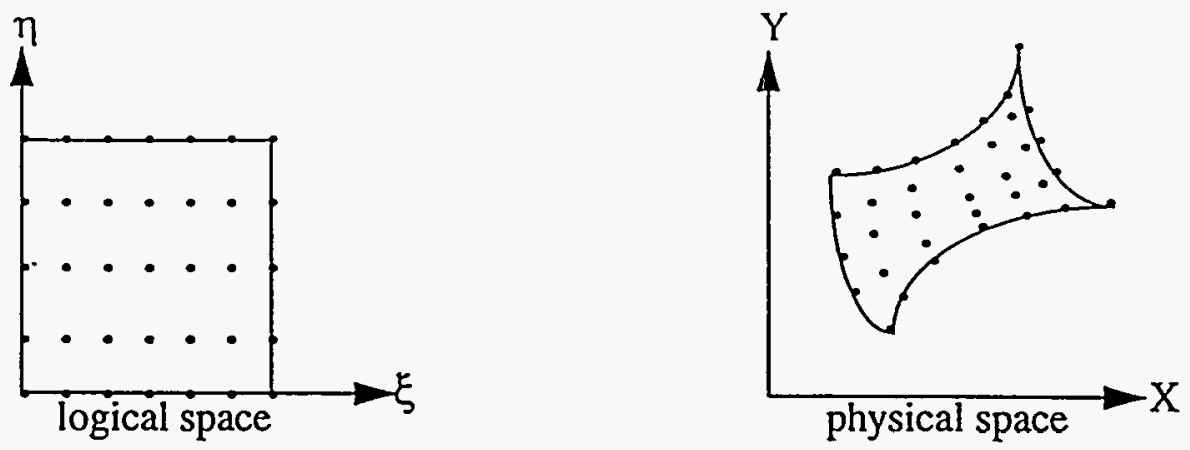

Fig. 2: Points mapped from Logical to Physical Space.

To restate, then, the maps, or transformations from logical space to physical space give coordinate systems in physical space whose coordinate lines are images of coordinate lines in the logical region, and whose boundaries are images of the boundaries in the logical region. These transformations take a grid in the logical space and transform this grid to the physical space.

Two situations must be avoided with the transformed grid. First, it is unacceptable to have a point in logical space be mapped to a point outside the physical region. Such a mapping results in a folded grid, as seen in Fig. 3 .

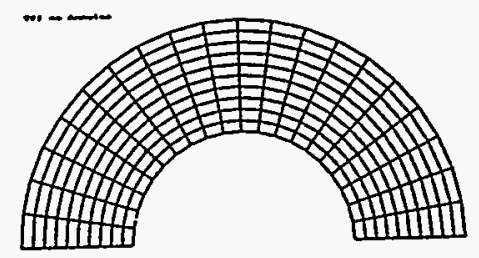

Initial Grid

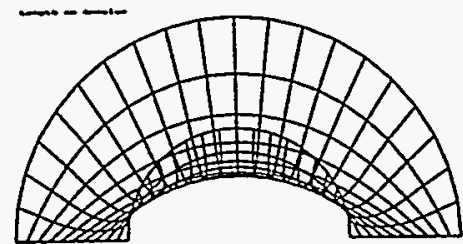

Resulting Folded Grid

Fig. 3: An Example of a Folded Grid.

Second, it is crucial that two or more points in logical space not be mapped to the same point in the physical region and vice versus. To avoid these problems, it is important to 
guarantee that the mapping is "one-to-one", such that each point in the logical space maps to a unique point in the physical space. Additionally, it is important to seek a mapping that is "onto", meaning that each point in the physical region is the image of a point in the logical space.

Consider the transformation

$$
\begin{aligned}
& x=x(\xi, \eta, \zeta) \\
& y=y(\xi, \eta, \zeta) \\
& z=z(\xi, \eta, \zeta) .
\end{aligned}
$$

The Jacobian matrix, $\mathbf{J}$, of this transformation is a square, and for three dimensions has the form

$$
\mathbf{J}=\left[\begin{array}{l}
\frac{\partial x}{\partial \xi} \frac{\partial x}{\partial \eta} \frac{\partial x}{\partial \zeta} \\
\frac{\partial y}{\partial \xi} \frac{\partial y}{\partial \eta} \frac{\partial y}{\partial \zeta} \\
\frac{\partial z}{\partial \xi} \frac{\partial z}{\partial \eta} \frac{\partial z}{\partial \zeta}
\end{array}\right]=\left[\begin{array}{ccc}
x_{\xi} & x_{\eta} & x_{\zeta} \\
y_{\xi} & y_{\eta} & y_{\zeta} \\
z_{\xi} & z_{\eta} & z_{\zeta}
\end{array}\right]
$$

Since the matrix is square, the determinant of $\mathbf{J}$ is

$$
j=\operatorname{det}(\mathbf{J}) \text {. }
$$

The "Inverse Mapping" Theorem ${ }^{19}$ relates the Jacobian of the transformation to the concept of "one-to-one":

Theorem: Assume a mapping exists whose coordinates are in the space of functions that have continuous derivatives. Then the mapping is one-to-one if and only if the determinant of the Jaco- 
bian, $j$, of the transformation is nonzero for all values $(\xi, \eta, \zeta)$ in the logical region.

Hence, to guarantee a one-to-one mapping, the transformation must yield a nonzero determinant of the Jacobian.

Elliptic grid generators use the solutions of systems of elliptic partial differential equations as their underlying transformation. As mentioned in the previous section, elliptic partial differential equations have several attractive properties that lend themselves well to generating smooth grids, most importantly that these solutions obey the extremum principle. However, an obvious concern is whether such solutions yield a nonzero determinant of the Jacobian, thus guaranteeing a "one-to-one" mapping. Combining both the extremum principle which states that solutions to elliptic partial differential equations obtain their maximum and minimum values on the boundary, and the one-toone mapping will produce a mesh that is both smooth and not folded ${ }^{34}$.

In the simplest case, suppose each component of the map, or transformation,

$$
\begin{aligned}
& x(\xi, \eta) \\
& y(\xi, \eta)
\end{aligned}
$$

satisfies the two dimensional Laplace's equation

$$
\begin{aligned}
& \nabla^{2} x=x_{\xi \xi}+x_{\eta \eta}=0 \\
& \nabla^{2} y=y_{\xi \xi}+y_{\eta \eta}=0
\end{aligned}
$$

with Dirichlet boundary conditions. The elliptic grid generator based on this transformation is called the AH (Amsden-Hirt) or Length generator ${ }^{1}$ and is used successfully in 
cases with convex regions. However, it can be shown ${ }^{19}$ that the transformation for this elliptic system gives a $\operatorname{det}(\mathbf{J})=0$, resulting in folded grids for non-convex regions. There is no guarantee that the mapping is "one-to-one".

The second technique is the most widely used elliptic grid generator, and is called the Winslow-Crowley or Equipotential method ${ }^{9,38,39}$, or the homogeneous ThompsonThames-Mastin (TTM) generator ${ }^{33}$. In two dimensions, this approach requires the components of the inverse transformation

$$
\begin{aligned}
& \xi=\xi(x, y) \\
& \eta=\eta(x, y)
\end{aligned}
$$

to satisfy Laplace's equation

$$
\begin{aligned}
& \nabla^{2} \xi=\xi_{x x}+\xi_{y y}=0 \\
& \nabla^{2} \eta=\eta_{x x}+\eta_{y y}=0
\end{aligned}
$$

The Dirichlet boundary conditions are given by the inverse of the physical boundaries. Note that the solutions to Laplace's equations are harmonic functions.The following theorem states that this inverse transformation produces a nonzero determinant of the $\mathrm{Jacobian}^{23}$, guaranteeing that the mapping is "one-to-one".

Theorem: If a simply connected region $D$ is mapped to a rectangular region $\mathrm{R}$ by the harmonic functions $\xi$ and $\eta$, then the transformation given by Eq. 10 has a nonvanishing determinant of the Jacobian on D.

The Winslow-Crowley algorithm is solved numerically by transforming Eq. 10 to 
logical space. It is assumed that in the transformation, $g \neq 0$, where $g$ is defined below, so that the mapping is invertible. The resulting inverted equation is

$$
\begin{aligned}
& g_{22} x_{\xi \xi}-2 g_{12} x_{\xi \eta}+g_{11} x_{\eta \eta}=0 \\
& g_{22} y_{\xi \xi}-2 g_{12} y_{\xi \eta}+g_{11} y_{\eta \eta}=0
\end{aligned}
$$

where

$$
\begin{aligned}
g_{11} & =x_{\xi}^{2}+y_{\xi}^{2} \\
g_{12}=g_{21} & =x_{\xi} x_{\eta}+y_{\xi} y_{\eta} \\
g_{22} & =x_{\eta}^{2}+y_{\eta}^{2}
\end{aligned}
$$

This mesh generating system tends to treat mesh lines as intersecting equipotentials, giving rise to its common nickname, the Equipotential method. This algorithm attempts to equally space the grid points because of the strong smoothing affect of the Laplacian. However, the resulting grid lines will become more closely spaced over convex boundaries and more sparsely spaced over concave boundaries ${ }^{34}$, as seen below.
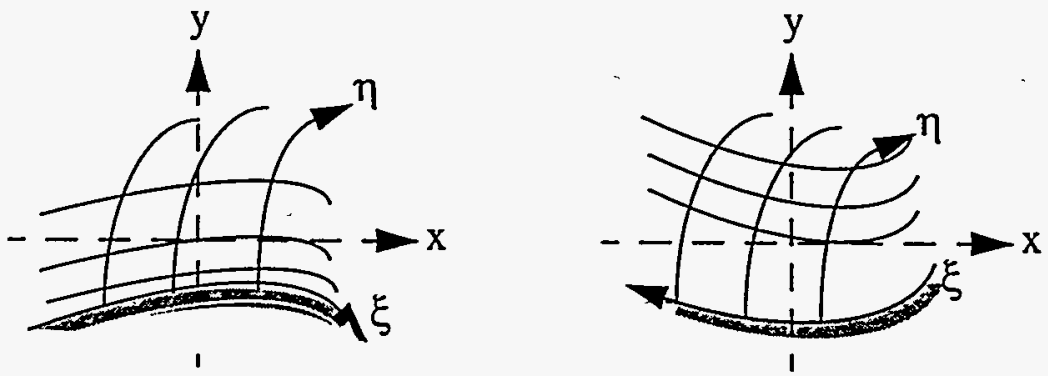

Fig. 4: Winslow-Crowley or homogeneous TTM at Concave and Convex surfaces.

One approach to controlling coordinate line distribution is to generalize the elliptic grid generating system to Poisson equations, sometimes called the inhomogeneous TTM generator $^{34}$ : 


$$
\begin{gathered}
\nabla^{2} \xi=\xi_{x x}+\xi_{y y^{\prime}}=P \\
\nabla^{2} \eta=\eta_{x x}+\eta_{y y}=Q
\end{gathered}
$$

in which $P$ and $Q$ are user specified weight functions used to attract or repel grid nodes toward specified regions in the logical domain. Although there is a theoretical basis for the Winslow-Crowley or homogeneous TTM method, there is no guarantee against folding or "one-to-one" mapping when the inhomogeneous terms are added. The theorem used to guarantee the "one-to-one" mapping for Laplace's equation does not hold for Poisson equations.

Another approach is to generalize Laplace's equation by including weight functions ${ }^{37}$ .This method, in two dimensions, requires the components of the inverse transformation

$$
\begin{aligned}
& \xi=\xi(x, y) \\
& \eta=\eta(x, y)
\end{aligned}
$$

to satisfy the weighted Làplace's equation

$$
\begin{aligned}
& \nabla \bullet(W \nabla \xi)=\frac{\partial}{\partial x}\left(W \frac{\partial \xi}{\partial x}\right)+\frac{\partial}{\partial y}\left(W \frac{\partial \xi}{\partial y}\right)=0 \\
& \nabla \bullet(W \nabla \eta)=\frac{\partial}{\partial x}\left(W \frac{\partial \eta}{\partial x}\right)+\frac{\partial}{\partial y}\left(W \frac{\partial \eta}{\partial y}\right)=0
\end{aligned}
$$

The user specified weight functions, $W$, are typically based on zonal quantities such as pressure and volume. These weight functions allow for some control over the placement of grid lines, and have been successful in certain fluid and continuum problems. The theorem, however, used to guarantee the "one-to-one" mapping for Laplace's equation does not hold for this weighted Laplace's equation.

Both the Winslow-Crowley or homogeneous TTM method and the inhomogeneous 
TTM generator have been extended to three dimensions $s^{31,35,37}$. The behaviors exhibited in two dimensions also occur in three dimensions, and the guaranteed "one-to-one" mapping for Laplace's equation still holds.

As the problems to be analyzed in fluid and continuum mechanics become more sophisticated, the need to control the mesh line placement becomes more important. The use of Poisson equations for mesh generators has been marginally successful, although choosing $P$ and $Q$, the user specified weight functions, is not trivial. A more robust approach is necessary. Beginning in the early 1980 's, elliptic grid generators derived using the Calculus of Variations approach emerged ${ }^{3}$, allowing for more versatile control of the grid line placement. The next section provides a review of the basic ideas behind the Calculus of Variations, followed by an analysis of several of the popular elliptic grid generators based on this approach.

\section{Calculus of Variations}

The Calculus of Variations ${ }^{2}$ involves problems in which the quantity to be minimized (or maximized) appears as an integral. Consider

$$
I=\int_{x_{1}}^{x_{2}} f\left(y, y_{x}, x\right) d x
$$

where the functional $I$ is the quantity that takes on an extremum, $f$ is a known function of the variables $y(x), y_{x}(x) \equiv \frac{d}{d x} y(x)$ and $x$, but $y(x)$ is unknown. So, although the integral is from $\left(x_{1}, y_{1}\right)$ to $\left(x_{2}, y_{2}\right)$, the exact path of integration is not known, as shown by the 
two example paths illustrated in Fig. 5.

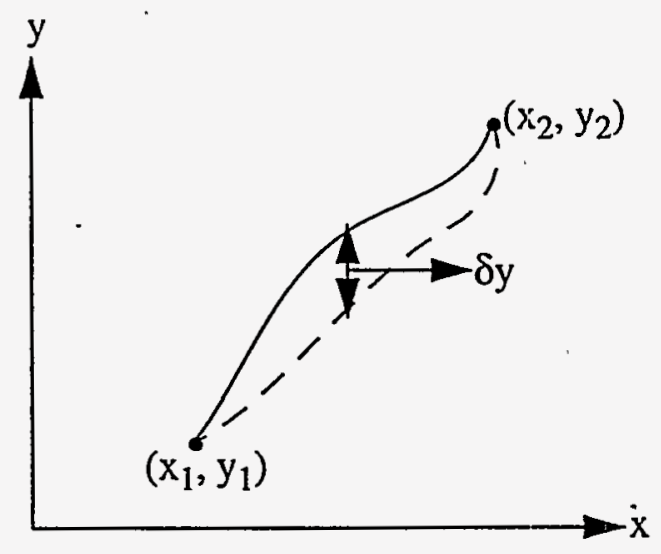

Fig. 5: A varied path.

The problem, then, is to find a function $y(x)$ that minimizes the functional $I$, assuming that there exists an optimum path. The difference between two paths for a given $x$ is called the variation of $y$, or $\delta y$. To define the arbitrary deformation of the path, the function $\eta(x)$ and a scale factor $\alpha$ are introduced to give the magnitude of the variation. The function $\eta(x)$ is arbitrary provided

$$
\eta\left(x_{1}\right)=\eta\left(x_{2}\right)=0
$$

which simply states that all varied paths must pass through the fixed endpoints, and $\eta(x)$ is differentiable. With the above definitions,

$$
y(x, \alpha)=y(x, 0)+\alpha \eta(x)
$$

and

$$
\delta y=y(x, \alpha)-y(x, 0)=\operatorname{c\eta \eta }(x)
$$

From Eq. 18 


$$
\frac{\partial}{\partial \alpha} y(x, \alpha)=\eta(x) \text { and } \frac{\partial}{\partial \alpha} y_{x}(x, \alpha)=\frac{d}{d x} \eta(x) .
$$

A one dimensional example with one dependent and one independent variable may prove instructive. First, $y(x, \alpha=0)$ is chosen as the unknown path that will minimize the functional $I$. Eq. 16 then becomes

$$
I(\alpha)=\int_{x_{1}}^{x_{2}} f\left[y(x, \alpha), y_{x}(x, \alpha), x\right] d x
$$

and the condition for an extreme value is that

$$
\left[\frac{\partial}{\partial x} I(\alpha)\right]_{\alpha=0}=0
$$

Taking the derivative of $I$ with respect to $\alpha$, and substituting in the definition of $\eta(x)$ from Eq. 20 gives

$$
\frac{\partial}{\partial \alpha} I(\alpha)=\int_{x_{1}}^{x_{2}}\left(\frac{\partial f}{\partial y} \eta+\frac{\partial f d \eta}{\partial y_{x} d x}\right) d x=0
$$

Integrating by parts and remembering that $\eta(x)$ is 0 at the endpoints gives

$$
\int_{x_{1}}^{x_{2}}\left[\frac{\partial f}{\partial y}-\frac{d}{d x}\left(\frac{\partial f}{\partial y_{x}}\right)\right] \eta d x=0 .
$$

Since $\eta(x)$ is arbitrary, the trivial solution $(\eta(x)=0)$ is ignored and an extremum can only be satisfied if the bracketed term itself in Eq. 24 is identically zero

$$
\frac{\partial f}{\partial y}-\frac{d}{d x}\left(\frac{\partial f}{\partial y_{x}}\right)=0
$$

which is known as the Euler equation, or Euler-Lagrange equation. This equation can be expressed in various other forms ${ }^{2}$. 
The calculus of variations approach can be extended to several dimensions. In the two dimensional case, with two dependent variables $\vec{x}=(x, y)$ and two independent variables $\vec{\xi}=(\xi, \eta)$, and the functional $I$ is defined as

$$
I[\grave{x}]=\int_{\xi} \int_{\eta} f\left(\xi, \eta, x, y, x_{\xi}, x_{\eta}, y_{\xi}, y_{\eta}\right) d \xi d \eta,
$$

resulting in the Euler-Lagrange equations below

$$
\begin{aligned}
& \frac{\partial f}{\partial x}-\frac{d}{d \xi}\left(\frac{\partial f}{\partial x_{\xi}}\right)-\frac{d}{d \eta}\left(\frac{\partial f}{\partial x_{\eta}}\right)=0 \\
& \frac{\partial f}{\partial y}-\frac{d}{d \xi}\left(\frac{\partial f}{\partial y_{\xi}}\right)-\frac{d}{d \eta}\left(\frac{\partial f}{\partial y_{\eta}}\right)=0
\end{aligned}
$$

Having outlined the basic principle of calculus of variations, the various mesh generation techniques based on this approach can be discussed.

\section{Variational Grid Generation}

The popularity of the elliptic grid generators, specifically the Winslow or TTM algorithms, arises primarily from their ability to give accurate, numerical representation of the boundary geometry. For example, in problems modeling the flow past an airfoil, correct representation of the boundary is critical, and the Winslow and TTM grid generators have proven to be affective ${ }^{3}$. Now consider problems in which strong gradients or singular areas develop in the interior of a region, as in reaction diffusion processes and shocked flows. In these problems, the resolution within the interior of the geometry is important, and mesh spacing control far from the boundary becomes just as critical as accurate representation of the geometry of the boundary. For these types of problems, the boundary conforming algorithms such as the Winslow or TTM algorithms lack the 
robustness needed to control the interior mesh representation.

The variational grid generation technique provides an approach to controlling the interior of meshes. The most widely known variational grid generator is presented by Brackbill and Saltzman ${ }^{3}$. Their approach consists of minimizing combinations of three different integrals, each of which controls some aspect of the mesh. In two dimensions, the smoothness of the mapping, $I_{s}$, is given by

$$
I_{s}=\int_{D}\left|(\nabla \xi)^{2}+(\nabla \eta)^{2}\right| d V
$$

The orthogonality, $I_{o}$, is measured by

$$
I_{o}=\int_{D}(\nabla \xi \cdot \nabla \eta)^{2} d V
$$

or the volume weighted measure

$$
I_{o}^{\prime}=\int_{D}(\nabla \xi \cdot \nabla \eta)^{2} j^{2} d V
$$

and the weighted volume variation, $I_{v}$ is measured by

$$
I_{v}=\int_{D} w j d V
$$

where $w=w(x, y)$ is a given function, with $j$ specified by Eq. 6 .

The smoothest mapping is obtained by minimizing $I_{s}$, the most orthogonal mapping by minimizing $I_{o}^{\prime}$, and the mapping with the specified variation of $j$ by minimizing $I_{v}$ These three integrals are combined to formulate minimization problems with unique solutions where

$$
I=I_{s}+\lambda_{v} I_{v}+\lambda_{o}^{\prime} I_{o}^{\prime}
$$


The numerical solution for this variational problem involves several steps. First, the Euler equations for each of the integrals in Eq. 32 are derived by transforming for convenience the integral to logical space. The indicated differentiation and collection of coefficients of highest derivatives is done, and finally, the discretization of the resulting equations is performed. For example, the transformation of the smoothness integral, $I_{s}$, results in

$$
I_{S}=\iint \frac{\left(x_{\xi}^{2}+x_{\eta}^{2}+y_{\xi}^{2}+y_{\eta}^{2}\right)}{j} d \xi d \eta
$$

and the corresponding Euler equations are

$$
\begin{aligned}
& \left(\frac{\partial}{\partial x}-\frac{\partial \partial}{\partial \xi \partial x_{\xi}}-\frac{\partial \partial}{\partial \eta \partial x_{\eta}}\right)\left(\frac{x_{\xi}^{2}+x_{\eta}^{2}+y_{\xi}^{2}+y_{\eta}^{2}}{j}\right)=0 \\
& \left(\frac{\partial}{\partial y}-\frac{\partial \partial}{\partial \xi \partial y_{\xi}}-\frac{\partial \partial \partial}{\partial \eta \partial y_{\eta}}\right)\left(\frac{x_{\xi}^{2}+x_{\eta}^{2}+y_{\xi}^{2}+y_{\eta}^{2}}{-j}\right)=0
\end{aligned}
$$

Performing the differentiation and collection of coefficients yields

$$
\begin{gathered}
B\left(\alpha x_{\xi \xi}-2 \beta x_{\xi \eta}+\gamma x_{\eta \eta}\right)-A\left(\alpha y_{\xi \xi}-2 \beta y_{\xi \eta}+\gamma y_{\eta \eta}\right)=0 \\
-A\left(\alpha x_{\xi \xi}-2 \beta x_{\xi \eta}+\gamma x_{\eta \eta}\right)+C\left(\alpha y_{\xi \xi}-2 \beta y_{\xi \eta}+\gamma y_{\eta \eta}\right)=0
\end{gathered}
$$

where 


$$
\begin{aligned}
& A=x_{\xi} y_{\xi}+x_{\eta} y_{\eta} \\
& B=y_{\xi}^{2}+y_{\eta}^{2} \\
& C=x_{\xi}^{2}+x_{\eta}^{2} \\
& \alpha=\frac{\left(x_{\eta}^{2}+y_{\eta}^{2}\right)}{j^{3}} \\
& \beta=\frac{\left(x_{\xi} x_{\xi}+y_{\eta} y_{\eta}\right)}{j^{3}} \\
& \gamma=\frac{\left(x_{\xi}^{2}+y_{\xi}^{2}\right)}{j^{3}}
\end{aligned}
$$

After the above approach had been done on all of the integrals in Eq. 32 , the mapping, or the resulting mesh is generated by finite difference approximations to these Euler equations, which are solved by iteration. Numerical solutions ${ }^{3}$ which minimize $I$ are obtained for finite values of $\lambda_{v}$ and $\lambda_{o}^{\prime}$, in which $\lambda_{v} \geq 0, \lambda_{o}^{\prime} \geq 0$.

Another variational approach is that of Steinberg and Roache ${ }^{28}$. It is interesting to note that the Brackbill and Saltzman theory and the Steinberg and Roache theory are basically equivalent ${ }^{5}$. The technique by Steinberg and Roache minimizes combinations of weighted length or smoothness, area, and orthogonality functionals,

$$
I_{W}=w_{s} I_{s}+w_{a} I_{a}+w_{o} I_{o}
$$

The weighted parameters $w_{s}, w_{a}$, and $w_{o}$ are nonnegative constants whose sum is one.

The length or smoothness functional, $I_{s}$, in two dimensions is given by

$$
I_{s}[\hat{x}]=\frac{1}{2} \int_{0}^{1} \int_{0}^{1}\left(\frac{g_{11}}{\phi(\xi, \eta)}+\frac{g_{22}}{\psi(\xi, \eta)}\right) d \xi d \eta
$$


where $\phi$ and $\psi$ are positive logical weight functions and $g_{I I}$ and $g_{22}$ are given by Eq. 12 .

The area functional, $I_{a}$, is specified by

$$
I_{a}[\grave{x}]=\frac{1}{2} \int_{0}^{1} \int_{0}^{1} \frac{g}{\varphi(\xi, \eta)} d \xi d \eta=\frac{1}{2} \int_{0}^{1} \int_{0}^{1} \frac{J^{2}}{\varphi(\xi, \eta)} d \xi d \eta
$$

The objective of minimizing this functional is to generate a grid such that the area of the cells in the grid are proportional to some given weight function $\varphi>0$. Recall that $g=j^{2}$ and that $j$, given by Eq. 6 is proportional to the area of the cell.

The orthogonality functional, $I_{o}$, is based on a different concept than the length and area functionals. The latter two were derived in an attempt to make specific quantities proportional, such as the length of the resulting mesh is proportional to $\phi$ and $\psi$, and the area proportional to $\varphi$. The orthogonality functional is based on trying to make a quantity zero. Recall that

$$
g_{12}=\left|\frac{d x}{d \xi}\right|\left|\frac{d x}{d \eta}\right| \cos \theta
$$

where $\theta$ is the angle between two tangent vectors to the grid lines. If the grid lines are orthogonal, $g_{12}=0$. An appropriate functional, then, is

$$
I_{o}[\vec{x}]=\frac{1}{2} \int_{0}^{1} \int_{0}^{1} g_{12}^{2} d \xi d \eta
$$

A special case of the Steinberg-Roache algorithm is the AO functional ${ }^{18}$, which produces an automatic grid generator by choosing the weighted parameters in Eq. 37 to be $w_{s}=0, w_{a}=\frac{1}{2}$, and $w_{o}=\frac{1}{2}$. The numerical solution of this technique is accom- 
plished in the same manner outlined with the BrackBill and Saltzman method.

The various grid generators presented in this section have proven useful for controlling the length of segments in the grid, areas of cells in the grid, and the orthogonality of the angles between grid lines. Except for the AO algorithm, which produces an automatic grid generator, the variational techniques presented above require user intervention. A user must set the various length, smoothness and orthogonality coefficients based on the specific problem. Of further concern, though, are attempts to use variational methods to generate grids on certain surfaces of modest shape which have failed ${ }^{29}$.

\section{Summary}

This chapter introduced the concept of grid generation, and presented the major algorithms currently being used. As mentioned earlier, the Winslow-Crowley or homogeneous TTM algorithm, perhaps the most widely used elliptic mesh generator, attempts to create cells of equal volume within the boundary. The major deficiency with this technique is the inability to control mesh lines. The use of variational techniques to generate elliptic mesh generators which allow for some control of mesh lines placement hàs proven fairly successful. The most widely known of these techniques is the Brackbill and Saltzman algorithm, which allows the user to control various grid parameters, such as angles between grid lines. Two major deficiencies of these techniques is the necessity for users to be knowledgable of the effects of these parameters, and the failure of these approaches when generating grids for problems with certain shapes.

Although this chapter concentrated on two dimensional grid generation algorithms, these techniques can be and have been applied to three dimensions in which similar results have been seen. 


\section{Chapter 3: Diffusive Mesh Relaxation}

With an understanding of the basics of mesh generation and various popular elliptic mesh generators from the previous chapter, a new mesh relaxer, the diffusive geometric mesh relaxer, can be presented. First, a brief review is provided of the general problem the mesh relaxation technique must solve, along with the motivation behind choosing a diffusive algorithm. Next, the variational formulization and the numerical solution of the algorithm are presented, and finally, the selection of the geometric diffusive coefficient is discussed.

\section{The Problem}

As mentioned in the Introductory chapter, the mesh relaxer must generate smooth grids while retaining the zoning structure of the original grid. In other words, coarse zoning in an area of the grid must remain coarse and fine zoning must remain fine. Additionally, the algorithm must automatically generate the appropriate mesh, requiring no user intervention.

The mesh relaxation technique developed here will be used in the remeshing step of an ALE procedure for solving the differential equations of continuum mechanics. The goal, then, of this dissertation is to derive a mesh relaxation method with the ability to adapt itself to the zoning structure of the existing mesh without user interaction.

\section{Motivation}

The idea to develop a mesh relaxation algorithm based upon the diffusion equation with a geometric diffusive weighting function came about from observing solutions to the heat transfer equation ${ }^{4}$. Heat transfer is the energy transfer which may take place 
between material bodies of different temperatures. When different parts of a body are at different temperatures, energy in the form of heat flows from the hotter parts to the cooler. One such method by which this transference occurs is conduction in which the heat passes through the body itself. The one-dimensional conservation of thermal energy equation $^{15}$, with terms for thermal diffusion and time dependent sources is

$$
\frac{\partial}{\partial x}\left(K \frac{\partial T}{\partial x}\right)+\dot{q}=\rho c \frac{\partial T}{\partial t}
$$

where

$$
\frac{\partial}{\partial x}\left(K \frac{\partial T}{\partial x}\right)
$$

is the diffusion term, $K$ the thermal conductivity, $\dot{q}$ the time dependent source term, and $\rho c \frac{\partial T}{\partial t}$ the transient term in which $\rho$ is the density and $c$ is the specific heat of the materials.

Eq. 42 reduces to

$$
\frac{\partial}{\partial x}\left(K \frac{\partial T}{\partial x}\right)=0
$$

for steady state problems in which $\frac{\partial T}{\partial t}=0$. Eq. 44 is a diffusive elliptic partial differential equation which obeys the three basic-principles discussed in Chapter 2 . To reiterate, elliptic partial differential equations yield solutions that are smooth, well behaved, and obey the extremum principle in which the maximum and minimum value attained by the solution occurs on the boundary of the problem in question. 
A one dimensional example will help in understanding the solutions to Eq. 42 and Eq. 44. Suppose two materials with differing thermal conductivities, $K_{I}$ and $K_{2}$, and initial temperatures, $T_{l}$ and $T_{2}$, are adjacent, as illustrated in Fig. 6

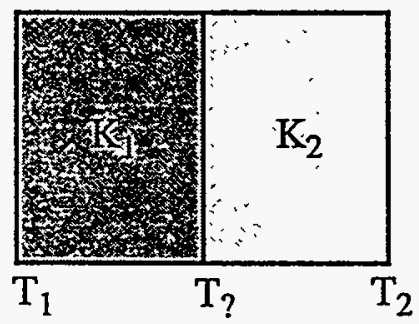

Fig. 6: Heat Transfer Example.

Eq. 42 and Eq. 44 try to determine the temperature, $T_{?}$, at the interface between these two materials and concurrently throughout the samples. For simplicity, set $T_{l}=100^{\circ} \mathrm{C}$ and $T_{2}=10^{\circ} \mathrm{C}$, and assume these boundaries remain fixed. A large thermal conductivity, $K$, implies that the material is a good conductor, whereas a small $K$ implies that the material does not conduct heat very well, and is, thus, a better insulator. For this example, set $K_{I}=202 \mathrm{~W}^{\circ} \mathrm{C} / \mathrm{m}$, as for aluminum, and $K_{2},=73 \mathrm{~W}^{\circ} \mathrm{C} / \mathrm{m}$, for iron. Due to the extremum principle, $T_{?}$ is between $T_{1}$ and $T_{2}$, and with the given values of $K_{1}$ and $K_{2}, T_{\text {? }}$ is much closer to $100^{\circ} \mathrm{C}$ than to $10^{\circ} \mathrm{C}$. Fig. 7 shows the isothermals determined by solv- 
ing Eq. 44 for this aluminum/iron example.

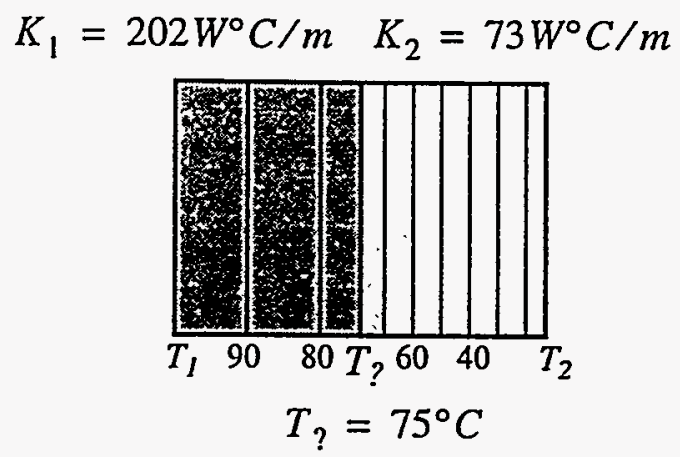

Fig. 7: Isothermals at $10^{\circ} \mathrm{C}$ for Heat Transfer Example.

$$
\left(T_{1}=100^{\circ} \mathrm{C}, T_{2}=10^{\circ} \mathrm{C}\right)
$$

An isothermal surface ${ }^{4}$ is one in which the temperature at every point along that surface is the same. Note that if $K_{I}$ and $K_{2}$ are reversed in the above example, $T_{\text {? }}$ is much closer to $10^{\circ} \mathrm{C}$ than $100^{\circ} \mathrm{C}$, and the isothermal spacing becomes more dense in material 1 than material 2. From the example described above, it is obvious that $T_{?}$ is very dependent on the values for $K_{1}$ and $K_{2}$. Thus, to achieve a particular $T_{?}$, the values of $K_{1}$ and $K_{2}$ need to be chosen appropriately.

From the influence $K_{1}$ and $K_{2}$ have on the temperatures calculated from the diffusion equation, it is easy to visualize a similar effect that a geometric parameter could have on a mesh calculated from a diffusive mesh relaxer. Suppose a one-dimensional mesh exists as in Fig. 8 for which $x_{?}$ is to be determined by the mesh relaxer.

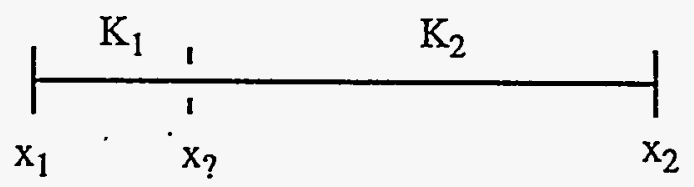

Fig. 8: Mesh Relaxation Example.

If it is desired that $x_{?}$ be close to $x_{1}$, then choose $K_{l}>K_{2}$. If it is desired that $x_{?}$ be close 
to $x_{2}$, then set $K_{1}<K_{2}$.

In a relaxation problem, $x$ ? is specified by the initial mesh. Assume for the moment that $x_{?}$ is set as shown in Fig. 8 . To maintain this existing zone structure, it is necessary that $K_{1}>K_{2}$ when applied to the diffusive mesh relaxer. If $K_{l}$ and $K_{2}$ are set to a geometric property, for example the inverse of the distance between the nodes,

$$
K_{1}=\frac{1}{\left(x_{9}-x_{1}\right)} \text { and } K_{2}=\frac{1}{\left(x_{2}-x_{?}\right)}
$$

then $K_{1}>K_{2}$. The existing zone structure is maintained, although $x_{\text {? }}$ might shift to the . right or left depending on how much greater $K_{I}$ is than $K_{2}$. The actual selection of $K_{1}$ and $K_{2}$ is discussed later in this chapter.

The motivation section illustrates how a diffusive relaxation technique can be used to address the problem requirements discussed in the previous section. Summarizing, a geometric diffusion coefficient acts like thermal conductivity when placed in a diffusion equation, and can be used to control the grid spacing of a given mesh, much like the thermal conductivity controls the spacing of the isotherms.

It is now appropriate to solve the diffusive mesh relaxer algorithm. The next sections discuss the numerical approximation of the diffusive mesh relaxation algorithm. The diffusive equation governing the mesh relaxation technique is first formulated in terms of a variation problem. The Galerkin finite element approach is then applied to this variational formulization. The Galerkin equations are then transformed from physical to logical space and finally, the basis functions and integrals for this transformed Galerkin finite element approximation are numerically evaluated. 


\section{Variational Formulization}

The first decision to make in specifying the diffusive mesh relaxer is to determine whether to require the diffusive elliptic equation be satisfied on the physical or logical mesh. Recall that in Chapter 2, the notion of a "one-to-one" mapping was introduced, and the existence of this criteria for several grid generation schemes was discussed. To reiterate, the $\mathrm{AH}$ grid generator which satisfied

$$
\begin{aligned}
& \nabla^{2} x=x_{\xi \xi}+x_{\eta \eta}=0 \\
& \nabla^{2} y=y_{\xi \xi}+y_{\eta \eta}=0
\end{aligned}
$$

did not satisfy the "one-to-one" mapping criteria, but the Winslow-Crowley or Equipotential method, or homogeneous TTM generator, which satisfied

$$
\begin{aligned}
\nabla^{2} \xi & =\xi_{x x}+\xi_{y y}=0 \\
\nabla^{2} \eta & =\eta_{x x}+\eta_{y y}=0
\end{aligned}
$$

did satisfy this criteria. Succeeding mesh generators, although unable to prove "one-toone" mapping, have also been applied to the physical mesh, as seen in Eq. 47 . The diffusive mesh relaxer will follow this same logic, and will require that

$$
\begin{aligned}
\xi & =\xi(x, y, z) \\
\eta & =\eta(x, y, z) \\
\zeta & =\zeta(x, y, z)
\end{aligned}
$$

satisfy the diffusive equations 


$$
\begin{aligned}
& \nabla \cdot\left(W_{1} \nabla \xi\right)=0 \\
& \nabla \bullet\left(W_{2} \nabla \eta\right)=0 \\
& \nabla \bullet\left(W_{3} \nabla \zeta\right)=0
\end{aligned}
$$

where

$$
W=\left[\begin{array}{l}
W_{1}(x, y, z) \\
W_{2}(x, y, z) \\
W_{3}(x, y, z)
\end{array}\right], \vec{\xi}=\left[\begin{array}{l}
\xi \\
\eta \\
\zeta
\end{array}\right] \text {, and } \vec{\xi}_{\text {boundary }} \neq 0
$$

The three diffusive equations specified in Eq. 49 will be solved to produce the new values for $(x, y, z)$, For simplicity, the equations in Eq. 49 will be referred to as

$$
\nabla \bullet(W \nabla \xi)=0
$$

with the forthcoming formulization applied identically to each of the three equations.

The governing equation for the diffusive mesh relaxer specified by Eq. 49 is restructured in terms of a variational problem. First, Eq. 49 is modified to have zero boundary conditions. Define a $v$ such that $v \neq \xi$, but $v_{\text {boundary }}=\xi_{\text {boundary }}$. If $\tilde{\xi}=\xi-v$, then $\tilde{\xi}_{\text {boundary }}=0$, and

$$
\nabla \cdot(W \nabla \tilde{\xi})=\nabla \bullet(W \nabla \xi)-\nabla \cdot(W \nabla v)=-\nabla \bullet(W \nabla v)=h
$$

due to Eq. 49 above. If Eq. 52 can be solved for $\tilde{\xi}$, then $\xi=\tilde{\xi}+v$ can be solved. Applying the variational principle outlined in Chapter 2 to Eq. 52 gives

$$
\iiint[\nabla \bullet(W \nabla \tilde{\xi})-h] w d x^{3}=0
$$

or 


$$
\iiint[\nabla \cdot(W \nabla \tilde{\xi})] w d x^{3}=\iiint h w d x^{3},
$$

where $w=w(x, y, z) \in H^{\prime}$ is arbitrary and $H^{\prime}$ is a Hilbert Space such that $w=0$ at the boundaries. Applying one of Green's Identities to both sides of Eq. 54 and substituting $-\nabla \cdot(W \nabla v)=h$ from Eq. 52 yields the following equation

$$
\iiint W \nabla \tilde{\xi} \bullet \nabla w d x^{3}=-\iiint W \nabla v \bullet \nabla w d x^{3} .
$$

Eq. 55 is the variational formulization for the diffusive mesh relaxer.

\section{Galerkin Finite Element Formulization}

The method for numerically approximating the variation problem presented in Eq. 55 is the Galerkin ${ }^{6}$ finite element approach. A mesh with $N$ nodes is constructed on the domain for which the variational problem is to be applied. For each node, $\beta$, on that mesh, there exists a basis, or shape function, $N_{\beta}=N_{\beta}(x, y, z) \in H^{\prime}$, such that

$$
N_{\beta}=\left(\begin{array}{ll}
1 & \text { at } \beta \\
0 & \text { otherwise }
\end{array}\right)
$$

The Galerkin method sets $w=N_{\beta}$ in Eq. 55 . Additionally, $\tilde{\xi}$ can be represented as an expansion of these basis functions

$$
\tilde{\xi}=\sum_{\alpha} f_{\alpha} N_{\alpha}
$$

where $f_{\alpha}$ is the value of $\tilde{\xi}$ at node $\alpha$, and $\mathrm{N}_{\alpha}$ is the basis function at that node. Applying the above definitions to Eq. 55 gives 


$$
\sum_{\alpha} f_{\alpha}\left[\iiint W \nabla N_{\alpha} \bullet \nabla N_{\beta} d x^{3}\right]=\iiint W \nabla \nu \bullet \nabla N_{\beta} d x^{3}
$$

Remember that $v$ is an arbitrary function such that $\nu \neq \xi$, but $v_{\text {boundary }}=\xi_{\text {boundary }}$.

Let $v$ be defined as

$$
v=\left(\begin{array}{cl}
\xi_{\text {boundary }} \cdot N_{*} & \text { at the boundary } \\
0 & \text { otherwise }
\end{array}\right)
$$

where $N_{*}$ is the basis function defined at the boundary node *. Substituting Eq. 59 into Eq. 58 produces the Galerkin finite element equations for the diffusive mesh relaxer

$$
\begin{array}{ll}
\sum_{\alpha} f_{\alpha}\left[\iiint W \nabla N_{\alpha} \bullet \nabla N_{\beta} d x^{3}\right]=\iiint W \xi_{b o u n d a r y} \nabla N_{*} \bullet \nabla N_{\beta} d x^{3} & \text { at the boundary } \\
\sum_{\alpha} f_{\alpha}\left[\iiint W \nabla N_{\alpha} \bullet \nabla N_{\beta} d x^{3}\right]=0 & \text { otherwise }
\end{array}
$$

To simplify, define

$$
M_{\alpha \beta}=\iiint W \nabla N_{\alpha} \bullet \nabla N_{\beta} d x^{3}
$$

such that Eq. 60 can be simplified to

$$
\sum_{\alpha} f_{\alpha} M_{\alpha \beta}=\left(\begin{array}{cl}
\xi_{\text {boundary }} M_{* \beta} & \text { at the boundary } \\
0 & \text { otherwise }
\end{array}\right) .
$$

Next, Eq. 62 is restructured such that

$$
\sum_{\alpha} M_{\alpha \beta} f_{\alpha}=M_{\alpha \alpha} f_{\alpha}+\sum_{\beta \neq \alpha} M_{\alpha \beta} f_{\beta}=\left(\begin{array}{cl}
\xi_{\text {boundary }} M_{* \beta} & \text { at the boundary } \\
0 & \text { otherwise }
\end{array}\right) .
$$

The matrix equation given by Eq. 63 is solved iteratively by means of the Jacobi Iterative Method ${ }^{14}$. This method is the simplest iterative scheme, and is used primarily because no iteration matrix needs to be accumulated. Applying the Jacobi iteration 
method produces the following

$$
f_{\alpha}^{(m+1)}=\left(\begin{array}{cl}
\frac{1}{M_{\alpha \alpha}}\left(\xi_{\text {boundary }} M_{* \beta}-\sum_{\beta \neq \alpha} M_{\alpha \beta} f_{\beta}^{(m)}\right) & \text { at boundary } \\
\left(-\frac{1}{M_{\alpha \alpha}}\left(\sum_{\beta \neq \alpha} M_{\alpha \beta} f_{\beta}^{(m)}\right)\right) & \text { otherwise }
\end{array}\right),
$$

where $m$ is the iteration count. Then, in light of Eq. 49 , Eq. 63 yields three independent matrix equations that need to be solved.

\section{Coordinate Transformation}

Before proceeding any further with the discretization of the diffusive mesh relaxer specified by Eq. 60 , it is important to discuss the usage of this relaxer. This algorithm is to be applied to the first step of the advection phase of an ALE simulation with physical regions of complex shape. As discussed earlier in Chapter 2, the equations in the ALE simulation are solved on the physical domain, but when the Galerkin method is applied, the integrals over each element are evaluated by transforming to the logical domain, allowing for isoparametric finite element analysis. Since the application domain applies this approach, it is logical that the mesh relaxer behave similarly.

Consider the coordinate transformation described in Eq. 4 with the corresponding Jacobian matrix, $\mathrm{J}$, defined in Eq. 5 . Then,

$$
\begin{gathered}
\frac{\partial N_{\alpha}}{\partial x_{i}}=\sum_{j} \frac{\partial \xi_{j} \partial N_{\alpha}}{\partial x_{i_{-}} \partial \xi_{j}} \\
\tilde{g}_{j k}=\sum_{i} \frac{\partial \xi_{j} \partial \xi_{k}}{\partial x_{i} \partial x_{i}} \\
d x^{3}=\mathbf{J} d \xi^{3}
\end{gathered}
$$


where the indices, $i, j, k$, correspond to the three axes,

$$
\vec{x}=\left[\begin{array}{l}
x_{1} \\
x_{2} \\
x_{3}
\end{array}\right]=\left[\begin{array}{l}
x \\
y \\
z
\end{array}\right] \text { and } \vec{\xi}=\left[\begin{array}{l}
\xi_{1} \\
\xi_{2} \\
\xi_{3}
\end{array}\right]=\left[\begin{array}{l}
\xi \\
\eta \\
\zeta
\end{array}\right]
$$

Substituting the above equations into Eq. 61 gives

$$
\begin{aligned}
M_{\alpha \beta} & =\iiint W(\xi)\left[\sum_{i}\left(\sum_{j} \frac{\partial \xi_{j} \partial N_{\alpha}}{\partial x_{i} \partial \xi_{j}}\right)\right] \cdot\left[\sum_{i}\left(\sum_{k} \frac{\partial \xi_{k} \partial N_{\beta}}{\partial x_{i}} \frac{\partial \xi_{k}}{\partial}\right)\right] \mathbf{J} d \xi^{3} \\
& =\iiint W(\xi) \sum_{i, j, k}\left(\frac{\partial \xi_{j} \partial \xi_{k}}{\partial x_{i} \partial x_{i}} \cdot \frac{\partial N_{\alpha}}{\partial \xi_{j}} \frac{\partial N_{\beta}}{\partial \xi_{k}}\right) \mathbf{J} d \xi^{3}
\end{aligned}
$$

or

$$
M_{\alpha \beta}=\iiint W(\xi) \sum_{j, k}\left(\tilde{g}_{j k} \cdot \frac{\partial N_{\alpha} \partial N_{\beta}}{\partial \xi_{j}} \frac{\partial \xi_{k}}{J}\right) d \xi^{3}
$$

The Galerkin finite element equation for the diffusive mesh relaxer is specified by Eq. 64 with $M_{\alpha \beta}$ defined by Eq. 70 .

\section{Final Application of Galerkin Finite Element Approach}

Before proceeding, it is appropriate to define some finite element terminology. The basis, or shape function for node $\alpha$ on the logical regime is defined as 
$N_{\alpha}(\xi, \eta, \zeta)=H(\xi) G(\eta) F(\zeta)$, where $H, G$, and $F$ are defined in Fig. 9 .

$$
H\left(\xi-\xi_{\alpha}\right)=\left(\begin{array}{cc}
1-\xi & \left(\xi_{\alpha} \leq \xi \leq 1\right) \\
1+\xi & \left(-1 \leq \xi \leq \xi_{\alpha}\right)
\end{array}\right)
$$

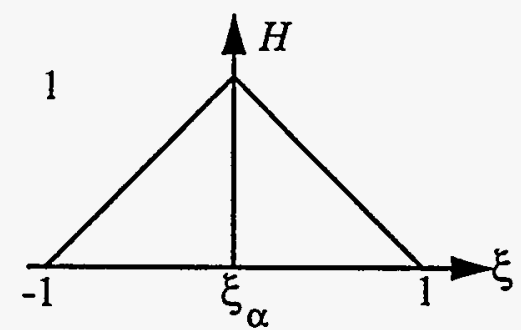

$$
G\left(\eta-\eta_{\alpha}\right)=\left(\begin{array}{cc}
1-\eta & \left(\eta_{\alpha} \leq \eta \leq 1\right) \\
1+\eta & \left(-1 \leq \eta \leq \eta_{\alpha}\right)
\end{array}\right)
$$

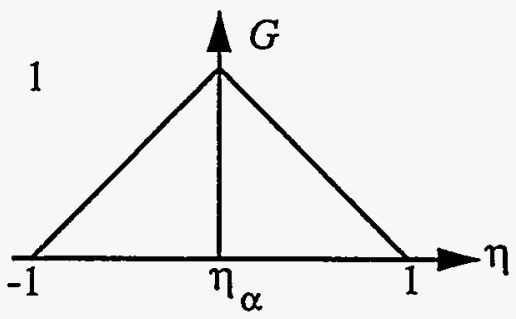

$$
F\left(\zeta-\zeta_{\alpha}\right)=\left(\begin{array}{cc}
1-\zeta & \left(\zeta_{\alpha} \leq \zeta \leq 1\right) \\
1+\zeta & \left(-1 \leq \zeta \leq \zeta_{\alpha}\right)
\end{array}\right)
$$

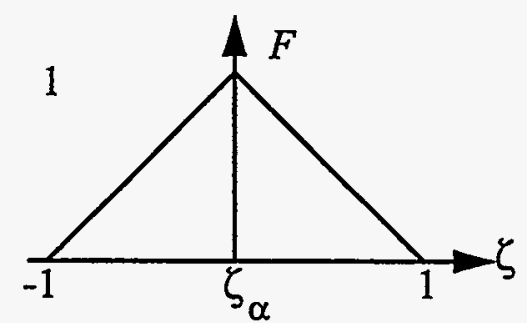

Fig. 9: Basis Functions

Using the definition of the basis functions described in Fig. 9 , the partial derivatives for these basis functions can be written as

$$
\begin{aligned}
& \frac{\partial N_{\alpha}}{\partial \xi}=\frac{\partial H}{\partial \xi} \bar{G} F=\left(\begin{array}{cc}
-G F & \left(\xi_{\alpha} \leq \xi \leq 1\right) \\
G F & \left(-1 \leq \xi \leq \xi_{\alpha}\right)
\end{array}\right) \\
& \frac{\partial N_{\alpha}}{\partial \eta}=\frac{\partial G}{\partial \eta} H F=\left(\begin{array}{cc}
-H F & \left(\eta_{\alpha} \leq \eta \leq 1\right) \\
H F & \left(-1 \leq \eta \leq \eta_{\alpha}\right)
\end{array}\right) \\
& \frac{\partial N_{\alpha}}{\partial \zeta}=\frac{\partial F}{\partial \zeta} H G=\left(\begin{array}{cc}
-H G & \left(\zeta_{\alpha} \leq \zeta \leq 1\right) \\
H G & \left(-1 \leq \zeta \leq \zeta_{\alpha}\right)
\end{array}\right) .
\end{aligned}
$$

Node $\alpha$ and its corresponding element, is made up of eight surrounding hexahedral elements, with the logical coordinate system having its origin at node $\alpha$, as shown in Fig. 
10.
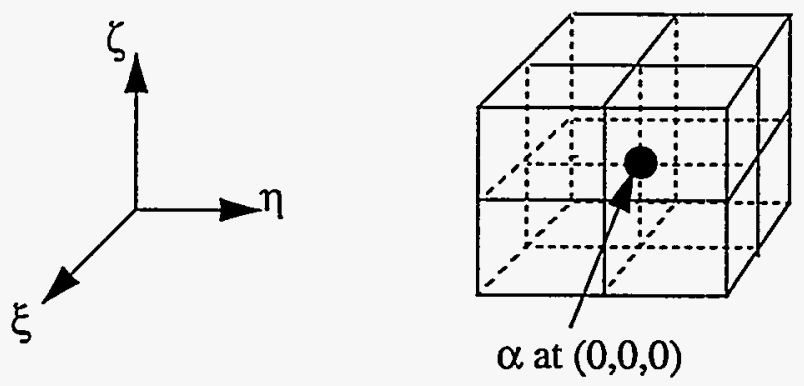

Fig. 10: Logical Coordinates and Node $\alpha$

Using node $\alpha$ defined in Fig. 10 and letting

$$
a_{i j}=j^{2} \tilde{g}_{i j} \text { and } w=\frac{W}{j},
$$

with $j=\operatorname{det}(J)$, Eq. 70 can be rewritten as

$$
M_{\alpha \beta}=\int_{-1}^{1} \int_{-1}^{1} \int_{-1}^{1} w \sum_{i, j=1}^{3} a_{i j}\left(\frac{\partial N_{\alpha}}{\partial \xi_{i}}\right)\left(\frac{\partial N_{\beta}}{\partial \xi_{j}}\right) d \xi^{3}
$$

and

$$
M_{\alpha \alpha}=\int_{-1}^{1} \int_{-1}^{1} \int_{-1}^{1} w \sum_{i, j=1}^{3} a_{i j}\left(\frac{\partial N_{\alpha}}{\partial \xi_{i}}\right)^{2} d \xi^{3}
$$

Expanding $\mathrm{M}_{\alpha \alpha}$ in Eq. 74 gives 


$$
\begin{gathered}
M_{\alpha \alpha}=\int_{-1}^{1} \int_{-1}^{1} \int_{-1}^{1} w\left(\sum_{i=1}^{3}\left(\frac{\partial N_{\alpha}}{\partial \xi_{i}}\right)^{2}\left(a_{i 1}+a_{i 2}+a_{i 3}\right) d \xi^{3}\right) \\
=\int_{-1}^{1} \int_{-1}^{1} \int_{-1}^{1} w\left(\left(\frac{\partial N_{\alpha}}{\partial \xi}\right)^{2}\left(a_{11}+a_{12}+a_{13}\right)\right. \\
+\left(\frac{\partial N_{\alpha}}{\partial \eta}\right)^{2}\left(a_{21}+a_{22}+a_{32}\right) \\
\left.+\left(\frac{\partial N_{\alpha}}{\partial \zeta}\right)^{2}\left(a_{31}+a_{32}+a_{33}\right)\right) d \xi^{3}
\end{gathered}
$$

For simplicity, define

$$
\begin{aligned}
& a_{1 j}=a_{11}+a_{12}+a_{13} \\
& a_{2 j}=a_{21}+a_{22}+a_{32} \\
& a_{3 j}=a_{31}+a_{32}+a_{33}
\end{aligned}
$$

but remember that $a_{i j}=a_{j i}, i \neq j$ from the definition of $a_{i j}$ in Eq. 72 and $\tilde{g}_{i j}$ from Eq. 66.

Since the basis functions are piecewise continuous in the element defined in Fig. 10 , the integral specified in Eq. 75 can be expanded to the following eight integrals 


$$
\begin{aligned}
M_{\alpha \alpha}= & \int_{-1}^{0} \int_{-1}^{0} \int_{-1}^{0} w\left(\left(\frac{\partial N_{\alpha}}{\partial \xi}\right)^{2} a_{1 j}+\left(\frac{\partial N_{\alpha}}{\partial \eta}\right)^{2} a_{2 j}+\left(\frac{\partial N_{\alpha}}{\partial \zeta}\right)^{2} a_{3 j}\right) d \xi^{3} \\
& +\int_{-1}^{0} \int_{-1}^{0} \int_{0}^{1} w\left(\left(\frac{\partial N_{\alpha}}{\partial \xi}\right)^{2} a_{1 j}+\left(\frac{\partial N_{\alpha}}{\partial \eta}\right)^{2} a_{2 j}+\left(\frac{\partial N_{\alpha}}{\partial \zeta}\right)^{2} a_{3 j}\right) d \xi^{3} \\
& +\int_{-1}^{0} \int_{0}^{1} \int_{-1}^{0} w\left(\left(\frac{\partial N_{\alpha}}{\partial \xi}\right)^{2} a_{1 j}+\left(\frac{\partial N_{\alpha}}{\partial \eta}\right)^{2} a_{2 j}+\left(\frac{\partial N_{\alpha}}{\partial \zeta}\right)^{2} a_{3 j}\right) d \xi^{3} \\
& +\int_{-1}^{0} \int_{0}^{1} \int_{0}^{1} w\left(\left(\frac{\partial N_{\alpha}}{\partial \xi}\right)^{2} a_{1 j}+\left(\frac{\partial N_{\alpha}}{\partial \eta}\right)^{2} a_{2 j}+\left(\frac{\partial N_{\alpha}}{\partial \zeta}\right)^{2} a_{3 j}\right) d \xi^{3} \\
& +\int_{0}^{1} \int_{-1}^{0} \int_{-1}^{0} w\left(\left(\frac{\partial N_{\alpha}}{\partial \xi}\right)^{2} a_{1 j}+\left(\frac{\partial N_{\alpha}}{\partial \eta}\right)^{2} a_{2 j}+\left(\frac{\partial N_{\alpha}}{\partial \zeta}\right)^{2} a_{3 j}\right) d \xi^{3} \\
& +\int_{0}^{1} \int_{-1}^{0} \int_{0}^{1} w\left(\left(\frac{\partial N_{\alpha}}{\partial \xi}\right)^{2} a_{1 j}+\left(\frac{\partial N_{\alpha}}{\partial \eta}\right)^{2} a_{2 j}+\left(\frac{\partial N_{\alpha}}{\partial \zeta}\right)^{2} a_{3 j}\right) d \xi^{3} \\
& +\int_{0}^{1} \int_{0}^{1} \int_{-1}^{0} w\left(\left(\frac{\partial N_{\alpha}}{\partial \xi}\right)^{2} a_{1 j}+\left(\frac{\partial N_{\alpha}}{\partial \eta}\right)^{2} a_{2 j}+\left(\frac{\partial N_{\alpha}}{\partial \zeta}\right)^{2} a_{3 j}\right) d \xi^{3} \\
& +\int_{0}^{1} \int_{0}^{1} \int_{0}^{1} w\left(\left(\frac{\partial N_{\alpha}}{\partial \xi}\right)^{2} a_{1 j}+\left(\frac{\partial N_{\alpha}}{\partial \eta}\right)^{2} a_{2 j}+\left(\frac{\partial N_{\alpha}}{\partial \zeta}\right)^{2} a_{3 j}\right) d \xi^{3}
\end{aligned}
$$

Using the partial derivatives for the basis functions defined by Eq. 71 , Eq. 77 can be further expanded to 


$$
\begin{aligned}
& M_{\alpha \alpha}=\int_{-1}^{0} \int_{-1}^{0} \int_{-1}^{0} w\left((1+\eta)^{2}(1+\zeta)^{2} a_{1 j}+(1+\xi)^{2}(1+\zeta)^{2} a_{2 j}+\right. \\
& \left.(1+\xi)^{2}(1+\eta)^{2} a_{3 j}\right) d \xi^{3} \\
& +\int_{-1}^{0} \int_{-1}^{0} \int_{0}^{1} w\left((1+\eta)^{2}(1-\zeta)^{2} a_{1 j}+(1+\xi)^{2}(1-\zeta)^{2} a_{2 j}+\right. \\
& \left.(1+\xi)^{2}(1+\eta)^{2} a_{3 j}\right) d \xi^{3} \\
& +\int_{-1}^{0} \int_{0}^{1} \int_{-1}^{0} w\left((1-\eta)^{2}(1+\zeta)^{2} a_{1 j}+(1+\xi)^{2}(1+\zeta)^{2} a_{2 j}+\right. \\
& \left.\quad(1+\xi)^{2}(1-\eta)^{2} a_{3 j}\right) d \xi^{3} \\
& +\int_{-1}^{0} \int_{0}^{1} \int_{0}^{1} w\left((1-\eta)^{2}(1-\zeta)^{2} a_{1 j}+(1+\xi)^{2}(1-\zeta)^{2} a_{2 j}+\right. \\
& +\int_{0}^{1} \int_{-1}^{0} \int_{-1}^{0} w\left((1+\eta)^{2}(1-\eta)^{2} a_{3 j}\right) d \xi^{3}(1+\zeta)^{2} a_{1 j}+(1-\xi)^{2}(1+\zeta)^{2} a_{2 j}+ \\
& \left.(1-\xi)^{2}(1+\eta)^{2} a_{3 j}\right) d \xi^{3} \\
& +\int_{0}^{1} \int_{-1}^{0} \int_{0}^{1} w\left((1+\eta)^{2}(1-\zeta)^{2} a_{1 j}+(1-\xi)^{2}(1-\zeta)^{2} a_{2 j}+\right. \\
& \left.(1-\xi)^{2}(1+\eta)^{2} a_{3 j}\right) d \xi^{3} \\
& +\int_{0}^{1} \int_{0}^{1} \int_{-1}^{0} w\left((1-\eta)^{2}(1+\zeta)^{2} a_{1 j}+(1-\xi)^{2}(1+\zeta)^{2} a_{2 j}+\right. \\
& +\int_{0}^{1} \int_{0}^{1} \int_{0}^{1} w\left((1-\xi)^{2}(1-\eta)^{2} a_{3 j}\right) d \xi^{3} \\
& \left.(1-\xi)^{2}(1-\zeta)^{2} a_{1 j}+(1-\eta)^{2} a_{3 j}\right) d \xi^{3}
\end{aligned}
$$

Next, these integrals in Eq. 78 are numerically evaluated using the second order integration scheme called the trapezoidal rule ${ }^{30}$, which states that for the integral

$$
I=\int_{\xi_{1}}^{\xi_{2}} \varphi(\xi) d \xi
$$

the trapezoidal rule yields 


$$
I=\left(\frac{\xi_{2}-\xi_{1}}{2}\right)\left[\varphi\left(\xi_{1}\right)+\varphi\left(\xi_{2}\right)\right]
$$

Eq. 80 indicates that the function, $\phi(\xi)$, is evaluated at the endpoints of the line interval from $\xi_{I}$ to $\xi_{2}$. In three dimensions, applying the trapezoidal rule along each direction of integration results in the function $\phi(\xi, \eta, \zeta)$ being evaluated around the volume surrounding the center of the element, namely node $\alpha$ as seen in Fig. 10 .

Numerically integrating the first integral in Eq. 78 using the trapezoidal rule in Eq. 80 gives

$$
\begin{aligned}
& \int_{-1}^{0} \int_{-1}^{0} \int_{-1}^{0} w\left((1+\eta)^{2}(1+\zeta)^{2} a_{1 j}+(1+\xi)^{2}(1+\zeta)^{2} a_{2 j}+\right. \\
& \left.(1+\xi)^{2}(1+\eta)^{2} a_{3 j}\right) d \xi^{3} \\
& =\int_{-1}^{0} \int_{-1}^{0} \frac{1}{2}\left[w_{i j(-1)}(1+\xi)^{2}(1+\eta)^{2} a_{3 j_{i j(-1)}}\right. \\
& \left.+w_{i j 0}\left((1+\eta)^{2} a_{1 j_{i j 0}}+(1+\xi)^{2} a_{2 j_{i j i}}+(1+\xi)^{2}(1+\eta)^{2} a_{3 j_{i j 0}}\right)\right] d \xi d \eta \\
& =\int_{-1}^{0} \frac{1}{4}\left[w_{i(-1) 0}(1+\xi)^{2} a_{2 j_{i(-1) 0}}+w_{i 0(-1)}(1+\xi)^{2} a_{3 j_{i t(-1)}}\right. \\
& \left.+w_{i 00}\left(a_{1 j_{i 00}}+(1+\xi)^{2} a_{2 j_{i 010}}+(1+\xi)^{2} a_{3 j_{i x 0}}\right)\right] d \xi \\
& =\frac{1}{8}\left[w_{-100} a_{1 j_{-100}}+w_{0(-1) 0} a_{2 j_{0(-1) 0}}+w_{00(-1)} a_{3 j_{00(-1)}}+\right.
\end{aligned}
$$

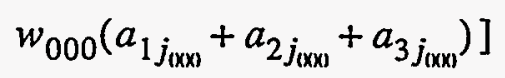

The remaining integrals in Eq. 78 follow similar evaluations, so $M_{\alpha \alpha}$ is numerically represented as 


$$
\begin{aligned}
& M_{\alpha \alpha}=\frac{1}{8}\left[w_{-100} a_{1 j_{-1(1)}}+w_{0-10} a_{2 j_{(1-10)}}+w_{00-1} a_{3 j_{(10-1}}+w_{000}\left(a_{1 j_{(x \times)}}+a_{2 j_{(x)}}+a_{3 j_{(x \times)}}\right)\right] \\
& +\frac{1}{8}\left[w_{-100} a_{1 j_{-1(\mathrm{x})}}+w_{0-10} a_{2 j_{(1-1)}}+w_{001} a_{3 j_{001}}+w_{000}\left(a_{1 j_{(\mathrm{xx})}}+a_{2 j_{(\mathrm{xx})}}+a_{3 j_{(x)}}\right)\right] \\
& +\frac{1}{8}\left[w_{-100} a_{1 j_{-1(x)}}+w_{010} a_{2 j_{0101}}+w_{00-1} a_{3 j_{(00-1}}+w_{000}\left(a_{1 j_{1 \times x)}}+a_{2 j_{(x \times)}}+a_{3 j_{(x)}}\right)\right] \\
& +\frac{1}{8}\left[w_{-100} a_{1 j_{-1(x)}}+w_{010} a_{2 j_{010}}+w_{001} a_{3 j_{(x) 1}}+w_{000}\left(a_{1 j_{(x)}}+a_{2 j_{(x \times 0)}}+a_{3 j_{(x \times)}}\right)\right] \\
& +\frac{1}{8}\left[w_{100} a_{1 j_{1(x)}}+w_{0-10} a_{2 j_{(1-1)}}+w_{00-1} a_{3 j_{(10-1}}+w_{000}\left(a_{1 j_{(x \times)}}+a_{2 j_{(x)}}+a_{3 j_{(x \times)}}\right)\right] \\
& +\frac{1}{8}\left[w_{100} a_{1 j_{1(x)}}+w_{0-10} a_{2 j_{(0)-1) 0}}+w_{001} a_{3 j_{(x) 1}}+w_{000}\left(a_{1 j_{(x \times)}}+a_{2 j_{(x) 0}}+a_{3 j_{(x \times)}}\right)\right] \\
& +\frac{1}{8}\left[w_{100} a_{1 j_{1(\mathrm{xx})}}+w_{010} a_{2 j_{0101}}+w_{00-1} a_{3 j_{(10-1}}+w_{000}\left(a_{1 j_{(\mathrm{xx})}}+a_{2 j_{(\mathrm{xx})}}+a_{3 j_{(\mathrm{xx})}}\right)\right] \\
& +\frac{1}{8}\left[w_{100} a_{1 j_{1(x)}}+w_{010} a_{2 j_{(010)}}+w_{001} a_{3 j_{(x) 1}}+w_{000}\left(a_{1 j_{(x \times)}}+a_{2 j_{(x) 0}}+a_{3 j_{(x \times)}}\right)\right] \\
& M_{\alpha \alpha}=\frac{1}{2}\left[w_{-100} a_{1 j_{-1(10)}}+w_{0-10} a_{2 j_{(1-10)}}+w_{00-1} a_{3 j_{0(1-1}}\right] \\
& +\frac{1}{2}\left[w_{100} a_{1 j_{1(x)}}+w_{010} a_{2 j_{010}}+w_{001}\right] a_{3 j_{(0) 1}}+w_{000}\left(a_{1 j_{(x \times)}}+a_{2 j_{(x)}}+a_{3 j_{(x \times)}}\right)
\end{aligned}
$$

where the notation $w_{i j k}$ stands for the variable $w$ at $\xi=\mathrm{i}, \eta=\mathrm{j}, \zeta=\mathrm{k}$ in Fig. 10 .

Expanding $M_{\alpha \beta}$ in Eq. 73 , using the elements and nodes surrounding node $\alpha$ in Fig. 10 and remembering that the basis functions are piecewise continuous over these elements, produces the following 26 integrals 
$M_{\alpha \beta}$

$=\int_{0}^{1} \int_{-1}^{0} \int_{-1}^{0} w \sum_{3^{i, j=1}}^{3} a_{i j}\left(\frac{\partial N_{\alpha}}{\partial \xi_{i}}\right)\left(\frac{\partial N_{\beta}}{\partial \xi_{j}}\right)_{1-1-1} d \xi^{3}+\int_{0}^{1} \int_{-1}^{1} \int_{-1}^{0} w \sum_{i, j=1}^{3} a_{i j}\left(\frac{\partial N_{\alpha}}{\partial \xi_{i}}\right)\left(\frac{\partial N_{\beta}}{\partial \xi_{j}}\right)_{10-1} d \xi^{3}$

$+\int_{0}^{1} \int_{0}^{1} \int_{-1}^{0} w \sum_{i, j=1}^{3^{i, j}=1} a_{i j}\left(\frac{\partial N_{\alpha}}{\partial \xi_{i}}\right)\left(\frac{\partial N_{\beta}}{\partial \xi_{j}}\right)_{11-1} d \xi^{3}+\int_{0}^{1} \int_{-1}^{0} \int_{-1}^{1} w \sum_{i, j=1}^{3} a_{i j}\left(\frac{\partial N_{\alpha}}{\partial \xi_{i}}\right)\left(\frac{\partial N_{\beta}}{\partial \xi_{j}}\right)_{1-10} d \xi^{3}$

$+\int_{0}^{1} \int_{-1}^{1} \int_{-1}^{1} w \sum_{i, j=1}^{3} a_{i j}\left(\frac{\partial N_{\alpha}}{\partial \xi_{i}}\right)\left(\frac{\partial N_{\beta}}{\partial \xi_{j}}\right)_{100} d \xi^{3}+\int_{0}^{1} \int_{0}^{1} \int_{-1}^{1} w \sum_{i, j=1}^{3} a_{i j}\left(\frac{\partial N_{\alpha}}{\partial \xi_{i}}\right)\left(\frac{\partial N_{\beta}}{\partial \xi_{j}}\right)_{110} d \xi^{3}$

$+\int_{0}^{1} \int_{-1}^{0} \int_{0}^{1} w \sum_{i, j=1}^{3} a_{i j}\left(\frac{\partial N_{\alpha}}{\partial \xi_{i}}\right)\left(\frac{\partial N_{\beta}}{\partial \xi_{j}}\right)_{1-11} d \xi^{3}+\int_{0}^{1} \int_{-1}^{1} \int_{0}^{1} w \sum_{i, j=1}^{3} a_{i j}\left(\frac{\partial N_{\alpha}}{\partial \xi_{i}}\right)\left(\frac{\partial N_{\beta}}{\partial \xi_{j}}\right)_{101} d \xi^{3}$

$+\int_{0}^{1} \int_{0}^{1} \int_{0}^{1} w \sum_{i, j=1}^{3} a_{i j}\left(\frac{\partial N_{\alpha}}{\partial \xi_{i}}\right)\left(\frac{\partial N_{\beta}}{\partial \xi_{j}}\right)_{111} d \xi^{3}+\int_{-1}^{1} \int_{-1}^{0} \int_{-1}^{0} w \sum_{i, j=1}^{3} a_{i j}\left(\frac{\partial N_{\alpha}}{\partial \xi_{i}}\right)\left(\frac{\partial N_{\beta}}{\partial \xi_{j}}\right)_{0-1-1} d \xi^{3}$

$+\int_{-1}^{1} \int_{-1}^{1} \int_{-1}^{0} w \sum_{i, j=1}^{3} a_{i j}\left(\frac{\partial N_{\alpha}}{\partial \xi_{i}}\right)\left(\frac{\partial N_{\beta}}{\partial \xi_{j}}\right)_{00-1} d \xi^{3}+\int_{-1}^{1} \int_{0}^{1} \int_{-1}^{0} w \sum_{i, j=1}^{3} a_{i j}\left(\frac{\partial N_{\alpha}}{\partial \xi_{i}}\right)\left(\frac{\partial N_{\beta}}{\partial \xi_{j}}\right)_{01-1} d \xi^{3}$

$+\int_{-1}^{1} \int_{-1}^{0} \int_{-1}^{1} w \sum_{i, j=1}^{3} a_{i j}\left(\frac{\partial N_{\alpha}}{\partial \xi_{i}}\right)\left(\frac{\partial N_{\beta}}{\partial \xi_{j}}\right)_{0-10} d \xi^{3}+\int_{-1}^{1} \int_{0}^{1} \int_{-1}^{1} w \sum_{i, j=1}^{3} a_{i j}\left(\frac{\partial N_{\alpha}}{\partial \xi_{i}}\right)\left(\frac{\partial N_{\beta}}{\partial \xi_{j}}\right)_{010} d \xi^{3}$

$+\int_{-1}^{1} \int_{-1}^{0} \int_{0}^{1} w \sum_{i, j=1}^{3} a_{i j}\left(\frac{\partial N_{\alpha}}{\partial \xi_{i}}\right)\left(\frac{\partial N_{\beta}}{\partial \xi_{j}}\right)_{0-11} d \xi^{3}+\int_{-1}^{1} \int_{-1}^{1} \int_{0}^{1} w \sum_{i, j=1}^{3} a_{i j}\left(\frac{\partial N_{\alpha}}{\partial \xi_{i}}\right)\left(\frac{\partial N_{\beta}}{\partial \xi_{j}}\right)_{001} d \xi^{3}$

$+\int_{-1}^{1} \int_{0}^{1} \int_{0}^{1} w \sum_{i, j=1}^{3} a_{i j}\left(\frac{\partial N_{\alpha}}{\partial \xi_{i}}\right)\left(\frac{\partial N_{\beta}}{\partial \xi_{j}}\right)_{011} d \xi^{3}+\int_{-1}^{0} \int_{-1}^{0} \int_{-1}^{0} w \sum_{i, j=1}^{3} a_{i j}\left(\frac{\partial N_{\alpha}}{\partial \xi_{i}}\right)\left(\frac{\partial N_{\beta}}{\partial \xi_{j}}\right)_{-1-1-1} d \xi^{3}$

$+\int_{-1}^{0} \int_{-1}^{1} \int_{-1}^{0} w \sum_{i, j=1}^{3} a_{i j}\left(\frac{\partial N_{\alpha}}{\partial \xi_{i}}\right)\left(\frac{\partial N_{\beta}}{\partial \xi_{j}}\right)_{-10-1} d \xi^{3}+\int_{-1}^{0} \int_{0}^{1} \int_{-1}^{0} w \sum_{i, j=1}^{3} a_{i j}\left(\frac{\partial N_{\alpha}}{\partial \xi_{i}}\right)\left(\frac{\partial N_{\beta}}{\partial \xi_{j}}\right)_{-11-1} d \xi^{3}$

$+\int_{-1}^{0} \int_{-1}^{0} \int_{-1}^{1} w \sum_{i, j=1}^{3} a_{i j}\left(\frac{\partial N_{\alpha}}{\partial \xi_{i}}\right)\left(\frac{\partial N_{\beta}}{\partial \xi_{j}}\right)_{-1-10} d \xi^{3}+\int_{-1}^{0} \int_{-1}^{1} \int_{-1}^{1} w \sum_{i, j=1}^{3} a_{i j}\left(\frac{\partial N_{\alpha}}{\partial \xi_{i}}\right)\left(\frac{\partial N_{\beta}}{\partial \xi_{j}}\right)_{-100} d \xi^{3}$

$+\int_{-1}^{0} \int_{0}^{1} \int_{-1}^{1} w \sum_{i, j=1}^{3} a_{i j}\left(\frac{\partial N_{\alpha}}{\partial \xi_{i}}\right)\left(\frac{\partial N_{\beta}}{\partial \xi_{j}}\right)_{-110} d \xi^{3}+\int_{-1}^{0} \int_{-1}^{0} \int_{0}^{1} w \sum_{i, j=1}^{3} a_{i j}\left(\frac{\partial N_{\alpha}}{\partial \xi_{i}}\right)\left(\frac{\partial N_{\beta}}{\partial \xi_{j}}\right)_{-1-11} d \xi^{3}$

$+\int_{-1}^{0} \int_{-1}^{1} \int_{0}^{1} w \sum_{i, j=1}^{3} a_{i j}\left(\frac{\partial N_{\alpha}}{\partial \xi_{i}}\right)\left(\frac{\partial N_{\beta}}{\partial \xi_{j}}\right)_{-101} d \xi^{3}+\int_{-1}^{0} \int_{0}^{1} \int_{0}^{1} w \sum_{i, j=1}^{3} \cdot a_{i j}\left(\frac{\partial N_{\alpha}}{\partial \xi_{i}}\right)\left(\frac{\partial N_{\beta}}{\partial \xi_{j}}\right)_{-111} d \xi^{3}$

Evaluating the first integral in Eq. 83 gives 


$$
\begin{aligned}
& I_{1}=\int_{0}^{1} \int_{-1}^{0} \int_{-1}^{0} w \sum_{i, j=1}^{3} a_{i j}\left(\frac{\partial N_{\alpha}}{\partial \xi_{i}}\right)\left(\frac{\partial N_{\beta}}{\partial \xi_{j}}\right)_{1-1-1} d \xi^{3} \\
& =\int_{0}^{1} \int_{-1}^{0} \int_{-1}^{0} w \sum_{i=1}^{3}\left[\dot{a}_{i 1}\left(\frac{\partial N_{\alpha}}{\partial \xi_{i}}\right)\left(\frac{\partial N_{\beta}}{\partial \xi^{2}}\right)_{1-1-1}+a_{i 2}\left(\frac{\partial N_{\alpha}}{\partial \xi_{i}}\right)\left(\frac{\partial N_{\beta}}{\partial \eta}\right)_{1-1-1}+\right. \\
& \left.a_{i 3}\left(\frac{\partial N_{\alpha}}{\partial \xi_{i}}\right)\left(\frac{\partial N_{\beta}}{\partial \zeta}\right)_{1-1-1}\right] d \xi^{3} \\
& =\int_{0}^{1} \int_{-1}^{0} \int_{-1}^{0} w\left[a_{11}\left(\frac{\partial N_{\alpha}}{\partial \xi}\right)\left(\frac{\partial N_{\beta}}{\partial \xi}\right)_{1-1-1}+a_{12}\left(\frac{\partial N_{\alpha}}{\partial \xi}\right)\left(\frac{\partial N_{\beta}}{\partial \eta}\right)_{1-1-1}+\right. \\
& a_{13}\left(\frac{\partial N_{\alpha}}{\partial \xi}\right)\left(\frac{\partial N_{\beta}}{\partial \zeta}\right)_{1-1-1}+a_{21}\left(\frac{\partial N_{\alpha}}{\partial \eta}\right)\left(\frac{\partial N_{\beta}}{\partial \xi}\right)_{1-1-1}+a_{22}\left(\frac{\partial N_{\alpha}}{\partial \eta}\right)\left(\frac{\partial N_{\beta}}{\partial \eta}\right)_{1-1-1}+ \\
& a_{23}\left(\frac{\partial N_{\alpha}}{\partial \eta}\right)\left(\frac{\partial N_{\beta}}{\partial \zeta}\right)_{1-1-1}+a_{31}\left(\frac{\partial N_{\alpha}}{\partial \zeta}\right)\left(\frac{\partial N_{\beta}}{\partial \xi}\right)_{1-1-1}+ \\
& \left.a_{32}\left(\frac{\partial N_{\alpha}}{\partial \zeta}\right)\left(\frac{\partial N_{\beta}}{\partial \eta}\right)_{1-1-1}+a_{33}\left(\frac{\partial N_{\alpha}}{\partial \zeta}\right)\left(\frac{\partial N_{\beta}}{\partial \zeta}\right)_{1-1-1}\right] d \xi^{3} \\
& =\int_{0}^{1} \int_{-1}^{0} \int_{-1}^{0} w\left[\left(\frac{\partial N_{\alpha}}{\partial \xi}\right)\left\{a_{11}\left(\frac{\partial N_{\beta}}{\partial \xi}\right)_{1-1-1}+a_{12}\left(\frac{\partial N_{\beta}}{\partial \eta}\right)_{1-1-1}+a_{13}\left(\frac{\partial N_{\beta}}{\partial \zeta}\right)_{1-1-1}\right\}+\right. \\
& \left(\frac{\partial N_{\alpha}}{\partial \eta}\right)\left\{a_{21}\left(\frac{\partial N_{\beta}}{\partial \xi}\right)_{1-1-1}+a_{22}\left(\frac{\partial N_{\beta}}{\partial \eta}\right)_{1-1-1}+a_{23}\left(\frac{\partial N_{\beta}}{\partial \zeta}\right)_{1-1-1}\right\}+ \\
& \left.\left(\frac{\partial N_{\alpha}}{\partial \zeta}\right)\left\{a_{31}\left(\frac{\partial N_{\beta}}{\partial \xi}\right)_{1-1-1}+a_{32}\left(\frac{\partial N_{\beta}}{\partial \eta}\right)_{1-1-1}+a_{33}\left(\frac{\partial N_{\beta}}{\partial \zeta}\right)_{1-1-1}\right\}\right] d \xi^{3}
\end{aligned}
$$

where $\left(\frac{\partial N_{\beta}}{\partial \xi_{l}}\right)_{i, j, k}$ is evaluated at node $\beta$, which is defined by $\xi=\mathrm{i}, \eta=\mathrm{j}, \zeta=\mathrm{k}$ in Fig. 10

Substituting into Eq. 84 the partial derivatives for the basis functions defined in Eq.

71 yields 
50

$$
\begin{aligned}
I_{1}=\int_{0}^{1} \int_{-1}^{0} \int_{-1}^{0} w[ \\
\quad\{-(1+\eta)(1+\zeta)\}\left\{a_{11}(1-\eta)(1-\zeta)-a_{12}(1+\xi)(1-\zeta)-a_{13}(1+\xi)(1-\eta)\right\}+ \\
\quad\{(1-\xi)(1+\zeta)\}\left\{a_{21}(1-\eta)(1-\zeta)-a_{22}(1+\xi)(1-\zeta)-a_{23}(1+\xi)(1-\eta)\right\}+ \\
\left.\{(1-\xi)(1+\eta)\}\left\{a_{31}(1-\eta)(1-\zeta)-a_{32}(1+\xi)(1-\zeta)-a_{33}(1+\xi)(1-\eta)\right\}\right] d \xi^{3}
\end{aligned}
$$

The trapezoidal rule described in Eq. 80 is applied to Eq. 85 to obtain 


$$
\begin{aligned}
& I_{1}=\int_{0}^{1} \int_{-1}^{0}\left[\frac{w_{i j(-1)}}{2}\{(1-\xi)(1+\eta)\}\right. \\
& \left\{2 a_{31}(1-\eta)-2 a_{32}(1+\xi)-a_{33}(1+\xi)(1-\eta)\right\}+ \\
& \frac{w_{i j 0}}{2}\left\{-(1+\eta)\left\{a_{11}(1-\eta)-a_{12}(1+\xi)-a_{13}(1+\xi)(1-\eta)\right\}+\right. \\
& (1-\xi)\left\{a_{21}(1-\eta)-a_{22}(1+\xi)-a_{23}(1+\xi)(1-\eta)\right\}+ \\
& \left.\left.\{(1-\xi)(1+\eta)\}\left\{a_{31}(1-\eta)-a_{32}(1+\xi)-a_{33}(1+\xi)(1-\eta)\right\}\right\}\right] d \xi d \eta \\
& =\int_{0}^{1}\left[\frac{w_{i(-1) 0}}{4}(1-\xi)\left\{2 a_{21}-a_{22}(1+\xi)-2 a_{23}(1+\xi)\right\}+\right. \\
& \frac{w_{i 0(-1)}}{4}(1-\xi)\left\{2 a_{31}-2 a_{32}(1+\xi)-a_{33}(1+\xi)\right\}+ \\
& \frac{w_{i 00}}{4}\left\{-\left\{a_{11}-a_{12}(1+\xi)-a_{13}(1+\xi)\right\}+\right. \\
& (1-\xi)\left\{a_{21}-a_{22}(1+\xi)-a_{23}(1+\xi)\right\}+ \\
& \left.\left.(1-\xi)\left\{a_{31}-a_{32}(1+\xi)-a_{33}(1+\xi)\right\}\right\}\right] d \xi \\
& =\frac{w_{100}}{8}\left[-a_{11}+2 a_{12}+2 a_{13}\right]+\frac{w_{0(-1) 0}}{8}\left[2 a_{21}-a_{22}-2 a_{23}\right]+ \\
& \frac{w_{00(-1)}}{8}\left[2 a_{31}-2 a_{32}-a_{33}\right] \\
& \frac{w_{000}}{8}\left[-a_{11}+a_{12}+a_{13}+a_{21}-a_{22}-a_{23}+a_{31}-a_{32}-a_{33}\right]
\end{aligned}
$$

Before proceeding, it is worthwhile to examine $w_{000}$. The variable $w$ is the weight term applied between the node $\alpha$, and the corresponding nodes $\beta$. In the case of $w_{000}, \alpha$ $=\beta$, and hence,

$$
w_{000}=0
$$

There are six additional integrals in Eq. 83 that contribute to the same volume of integration as does $I_{l}$. Evaluating these six in a similar manner as $I_{l}$, and using Eq. 87, 
yields

$$
\begin{aligned}
& I_{2}=\frac{w_{100}}{8}\left[-a_{11}-2 a_{12}+2 a_{13}\right]+\frac{w_{0(-1) 0}}{8}\left[a_{22}\right]+\frac{w_{00(-1)}}{8}\left[2 a_{31}+2 a_{32}-a_{33}\right] \\
& I_{4}=\frac{w_{100}}{8}\left[-a_{11}+2 a_{12}-2 a_{13}\right]+\frac{w_{0(-1) 0}}{8}\left[2 a_{21}-a_{22}+2 a_{23}\right]+\frac{w_{00(-1)}}{8}\left[a_{33}\right] \\
& I_{5}=\frac{w_{100}}{8}\left[-a_{11}-2 a_{12}-2 a_{13}\right]+\frac{w_{0(-1) 0}}{8}\left[a_{22}\right]+\frac{w_{00(-1)}}{8}\left[a_{33}\right] \\
& I_{10}=\frac{w_{100}}{8}\left[a_{11}\right]+\frac{w_{0(-1) 0}}{8}\left[-2 a_{21}-a_{22}-2 a_{23}\right]+\frac{w_{00(-1)}}{8}\left[-2 a_{31}-2 a_{32}-a_{33}\right] \\
& I_{11}=\frac{w_{100}}{8}\left[a_{11}\right]+\frac{w_{0(-1) 0}}{8}\left[a_{22}\right]+\frac{w_{00(-1)}}{8}\left[-2 a_{31}+2 a_{32}-a_{33}\right] \\
& I_{13}=\frac{w_{100}}{8}\left[a_{11}\right]+\frac{w_{0(-1) 0}}{8}\left[-2 a_{21}-a_{22}+2 a_{23}\right]+\frac{w_{00(-1)}}{8}\left[a_{33}\right]
\end{aligned}
$$

Summing the six equations in Eq. 88 with $I_{l}$ in Eq. 86 gives

$$
I_{S_{1}}=\frac{w_{100}}{8}\left[-a_{11}\right]+\frac{w_{0(-1) 0}}{8}\left[-a_{22}\right]+\frac{w_{00(-1)}}{8}\left[-a_{33}\right]
$$

Applying the above techniques to the remaining integrals in Eq. 83 yields the same general format as in Eq. 89

$$
I_{S_{i}}=\frac{w_{\xi 00}}{8}\left[-a_{11}\right]+\frac{w_{0 \eta 0}}{8}\left[-a_{22}\right]+\frac{w_{00 \zeta}}{8}\left[-a_{33}\right]
$$

The numerical integration, then, of $f_{a}$ for an interior node $\alpha$ is 


$$
\begin{aligned}
f_{\alpha} & =-\frac{1}{-M_{\alpha \alpha}}\left(\sum_{\beta \neq \alpha} M_{\alpha \beta} f_{\beta}\right) \\
& =\frac{\sum_{\text {all contributing elements }}\left(\frac{w_{\xi 00}}{8}\left[-a_{11}\right]+\frac{w_{0 \eta 0}}{8}\left[-a_{22}\right]+\frac{w_{00 \zeta}}{8}\left[-a_{33}\right]\right) f_{\beta}}{\sum_{\text {all contributing elements }}\left(\frac{w_{\xi 00}}{8}\left[-a_{1 j}\right]+\frac{w_{0 \eta 0}}{8}\left[-a_{2 j}\right]+\frac{w_{00 \zeta}}{8}\left[-a_{3 j}\right]\right)} \\
& =\frac{\sum_{\text {all contributing elements }}\left(w_{\xi 00}\left[a_{11}\right]+w_{0 \eta 0}\left[a_{22}\right]+w_{00 \zeta}\left[a_{33}\right]\right) f_{\beta}}{\sum_{\left.w_{\xi 00}\left[a_{1 j}\right]+w_{0 \eta 0}\left[a_{2 j}\right]+w_{00 \zeta}\left[a_{3 j}\right]\right)}}
\end{aligned}
$$

When node $\alpha$ lies on the boundary, the numerical solution given in Eq. 91 has additional terms, as specified in Eq. 64 . The contributing elements consist of boundary nodes, with yet undefined basis functions. For example, examine a boundary node and its corresponding basis functions in one dimension, shown in Fig. 11 .
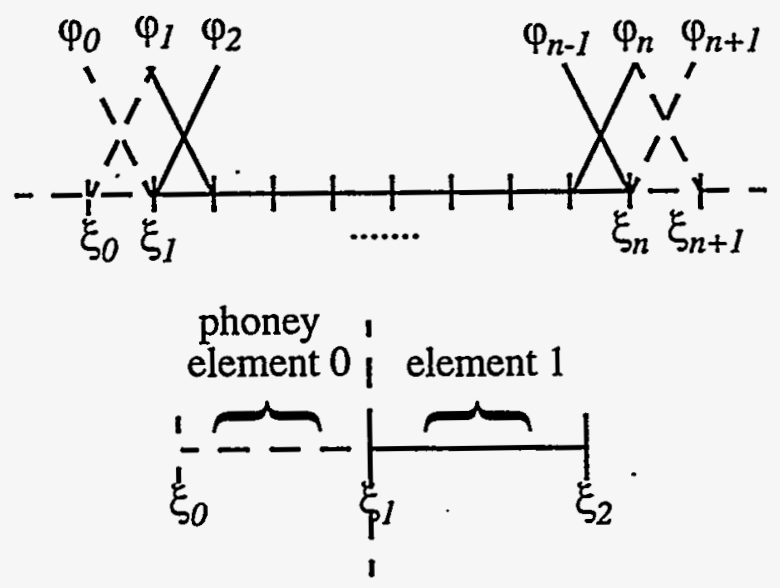

Fig. 11: One Dimensional Boundary Nodes and Their Corresponding Phoney Nodes The nodes $\xi_{1}$ and $\xi_{n}$ represent the boundary nodes, and $\varphi_{\alpha}$ represents the basis function at node $\alpha$. The phoney nodes $\xi_{0}$ and $\xi_{n+1}$ and their corresponding basis functions $\varphi_{0}$ and $\varphi_{n+l}$ are created by reflecting $\xi_{2}$ around $\xi_{1}$ to create $\xi_{0}$, and $\xi_{n-l}$ around $\xi_{n}$ to create $\xi_{n+1}$. Mathematically, given $\xi_{I}$ and $\xi_{2}, \xi_{0}$ can be derived by 


$$
x_{0}=2 x_{1}-x_{2}
$$

Applying Eq. 64 ,

$$
f_{\alpha}=\frac{1}{M_{\alpha \alpha}}\left(\xi_{\text {boundary }} M_{* \beta}-\sum_{\beta \neq \alpha} M_{\alpha \beta} f_{\beta}\right) \text { at boundary }
$$

For the boundary node $\xi_{I}$, in the one dimensional example given in Fig. 11 , define

$$
\begin{aligned}
& M_{\alpha \alpha}=M_{11}=\int_{x_{0}}^{x_{1}} w_{0} a_{0}\left(\frac{\partial \varphi_{1}}{\partial \xi}\right)\left(\frac{\partial \varphi_{1}}{\partial \xi}\right) d \xi+\int_{x_{1}}^{x_{2}} w_{1} a_{1}\left(\frac{\partial \varphi_{1}}{\partial \xi}\right)\left(\frac{\partial \varphi_{1}}{\partial \xi}\right) d \xi \\
& M_{\alpha \beta} f_{\alpha}=M_{12} f_{2}=\xi_{2} \int_{x_{1}}^{x_{2}} w_{1} a_{1}\left(\frac{\partial \varphi_{1}}{\partial \xi}\right)\left(\frac{\partial \varphi_{2}}{\partial \xi}\right) d \xi \\
& x_{\text {boundary }} M_{* \beta}=M_{10} f_{0}=\xi_{0} \int_{x_{0}}^{x_{1}} w_{0} a_{0}\left(\frac{\partial \varphi_{1}}{\partial \xi}\right)\left(\frac{\partial \varphi_{0}}{\partial \xi}\right) d \xi
\end{aligned}
$$

where $w_{i}$ and $a_{i}$ correspond to the values at element $i$, as seen in Fig. 11 . Since $\xi_{0}$ is the mirror image of $\xi_{2}$ with respect to $\xi_{1}$, and thus element 0 is the mirror image of element 1, let

$$
w_{0}=w_{1} \text { and } a_{0}=a_{1} \text {. }
$$

For the three dimensional case, the process applied above is repeated for each direction to create the phoney nodes, their elements, and the corresponding basis functions, which are mirror images of the existing nodes. The contributions resulting from these phoney elements are added to Eq. 91 .

\section{Determination of the Geometric Weight}

The geometric weight, $W$, is defined in such a manner as to control the amount of smearing or spreading of the grid. This is accomplished by analyzing the actual grid 
spacing between nodes in the $\mathrm{x}-, \mathrm{y}$ - and $\mathrm{z}$-directions.

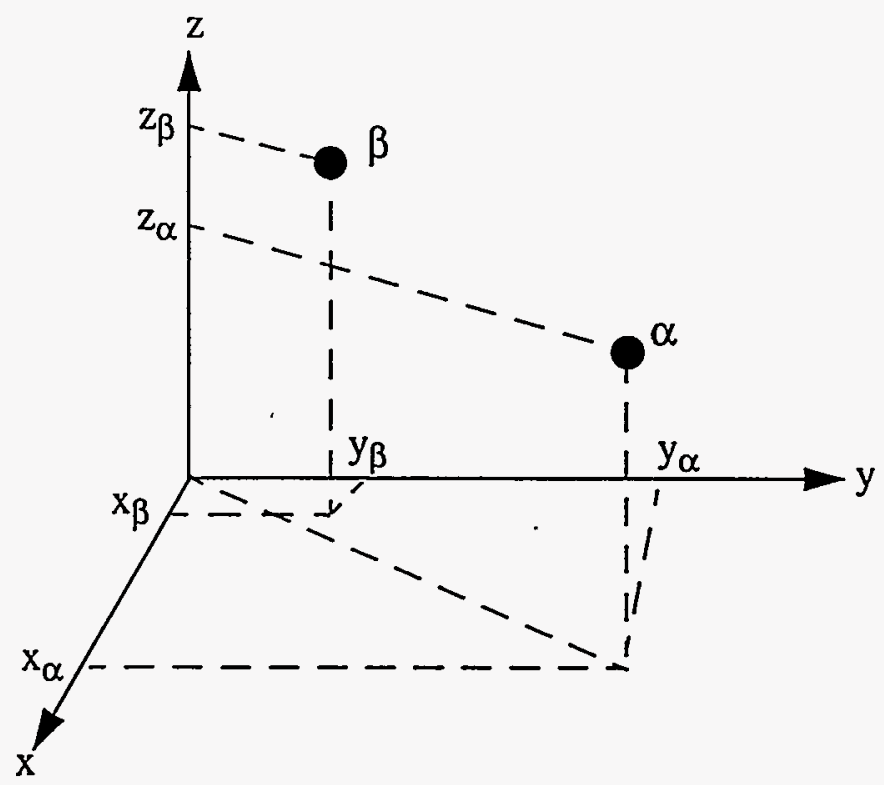

Fig. 12: Definition of Weighting Term

For example, when analyzing node $\alpha$ in Fig. 12 , the geometric weighting term between $\alpha$ and $\beta$ has the general form

$$
\begin{aligned}
& W_{x_{\alpha}}=\left(\frac{1}{|x(\alpha)-x(\beta)|}\right)^{r} \\
& W_{y_{\alpha}}=\left(\frac{1}{|y(\alpha)-y(\beta)|}\right)^{r} \\
& W_{z_{\alpha}}=\left(\frac{1}{|z(\alpha)-z(\beta)|}\right)^{r} .
\end{aligned}
$$

where $r$ is an arbitrary number. In the Applications chapter, several options for $r$ will be analyzed and the resulting effects to the mesh will be shown. 


\section{Chapter 4: Hourglass Control}

\section{Motivation}

The numerical solution to the diffusive mesh relaxation technique derived in the previous chapter is applied to a simple rectangular problem with zoning shown in Fig. 13 .

\begin{tabular}{|l|l|l|}
\hline & & \\
\hline & & \\
\hline & & \\
\hline & & \\
\hline & & \\
\hline & & \\
\hline
\end{tabular}

Fig. 13: Initial Mesh of Simple Rectangular Problem.

As the mesh relaxation algorithm iterates, an interesting phenomena develops with 
the mesh. Three consecutive iterations are displayed in Fig. 14 .
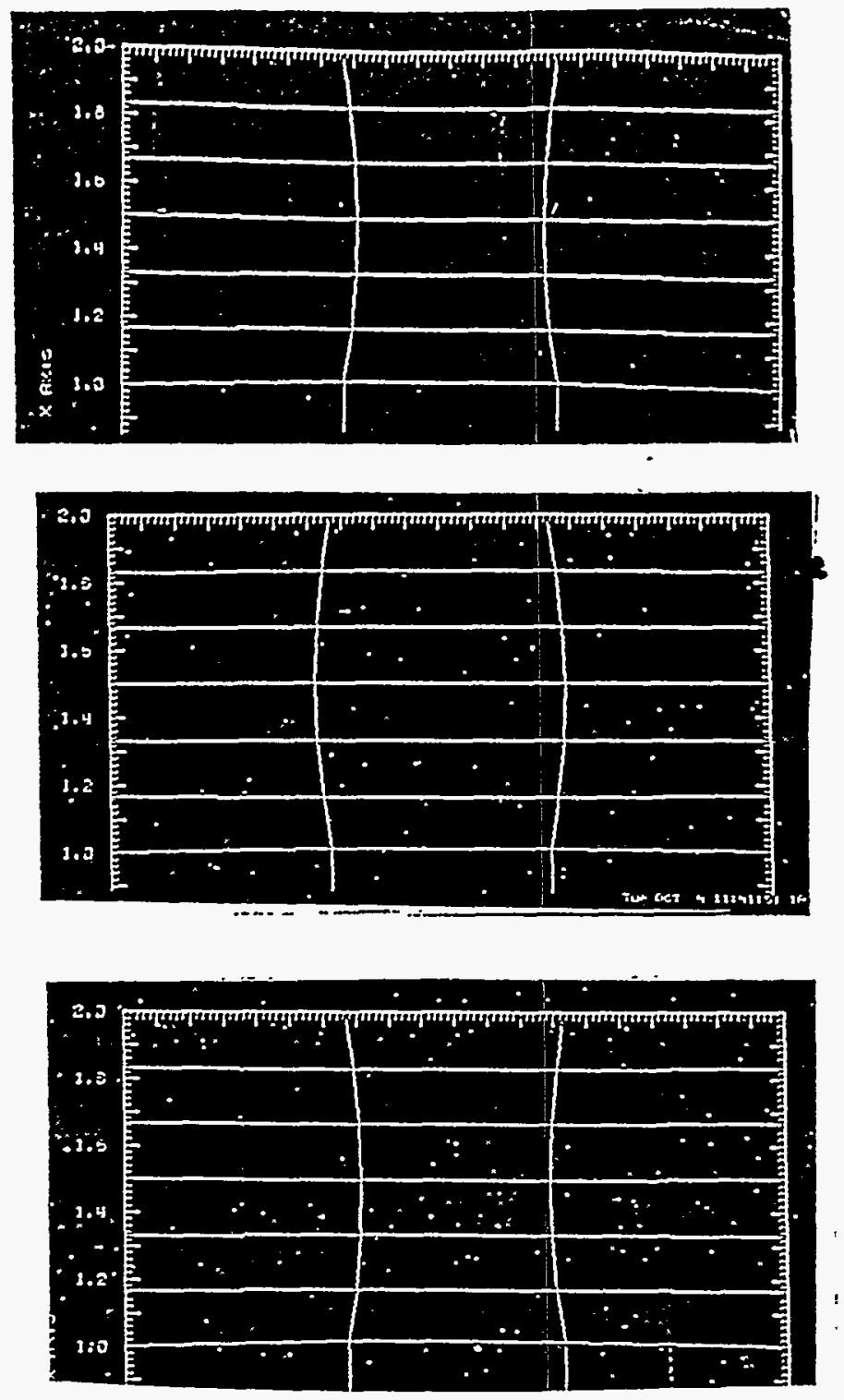

Fig. 14: Three Consecutive Iterations of Mesh for Simple Rectangular Problem.

Notice the fluctuations of the zones within the mesh: these zones alternate between two distinct sizes and shapes on consecutive iterations. This phenomena is known as hourglassing $6,11,20$.

In this Chapter, the term hourglassing is defined, and a simple two-dimensional example is used to illustrate the origins of the hourglass terms in the solution. Next, the 
method used for removing, or damping the hourglass phenomena from the diffusive mesh relaxer is described.

\section{Definition of Hourglassing}

One feature of finite element analysis is that any physical problem can be expressed as a system of matrix equations ${ }^{24}$. Consider a vibration problem in which the equations take the form of

$$
M\{\ddot{D}\}+C\{\dot{D}\}+K\{D\}=\{Q(t)\}
$$

where $\mathbf{M}, \mathbf{C}$, and $\mathbf{K}$ are respectively, the mass, damping and stiffness matrices for the complete system, and $\{\ddot{D}\},\{\dot{D}\}$ and $\{D\}$ are respectively the acceleration, velocity and displacement vectors for the system, with initial values at a number of points, or nodes in the system. Finally, $\{Q(t)\}$ is a vector of time dependent loads applied to these nodes. For an equilibrium problem, such as the equation governing the diffusive mesh generator, the matrix equation has the form

$$
K\{D\}=\{Q\}
$$

The first step in the finite element approach is to develop equations for each finite element, defining the necessary element matrices $m, c, k$, and the element vector $\{q\}$. Next, these element matrices are summed to form the matrices $M, C, K$ and the vector $\{Q\}$ for the complete domain,

$$
M=\sum m \quad C=\sum c \quad K=\sum k
$$

Once the matrices for the complete domain have been formed, the unknown nodal free- 
doms, shown in the following equilibrium example as the displacements $\{D\}$, are found by standard matrix solution techniques.

In the finite element formulization of equilibrium problems, whose form consists of that specified by Eq. 100 , the stiffness matrix $K$ consists of integrals that must be evaluated in solving the matrix equation. In many computer simulation codes, the most common approach for numerically solving these integrals in the stiffness matrix, $K$, is to use one-point integration rules. One-point integration schemes, however, for first-order finite elements results in certain unphysical deformation modes, called hourglass or zero energy modes. In dynamic codes, these modes often lead to instabilities in which displacements become unbounded.

Hourglass instability arises because the stiffness matrix is singular not only with respect to rigid body motion, but to hourglass patterns, and is non-singular only with respect to constant strain modes. The presence of hourglass modes, then, can be determined by examining the number of zero eigenvalues in the stiffness matrix ${ }^{6}$.

Several methods have been proposed for eliminating hourglass instabilities such as adding an hourglass response term ${ }^{11,20}$ to the one-point integration stiffness matrix without simultaneously stiffening the elements response to legitimate modes. Another obvious way to overcome the hourglass problem is to use a two-point quadrature scheme $^{20}$, but at a large increase in computational effort, especially in simulation codes for which the stiffness matrix is not stored but rather recalculated repeatedly. This is often the case in dynamic explicit codes and static iterative codes. Furthermore, it was pointed out by Wilson et al. ${ }^{40}$ that the isoparametric element with two-point quadrature 
does not accurately represent flexural modes of deformation.

\section{A Simple Two-Dimensional Example}

To demonstrate how these hourglass modes arise and are restrained, it is informative to initially study the behavior of a simple two-dimensional example: plane stress on an element. First, some terms will be defined and some background information provided, and then the stiffness matrix for the plane stress element is derived and analyzed.

Consider a four-node plane element. The eight independent displacement $\operatorname{modes}^{6}$, defined by $u$ and $v$, of this quadrilateral are shown in Fig. 15 .
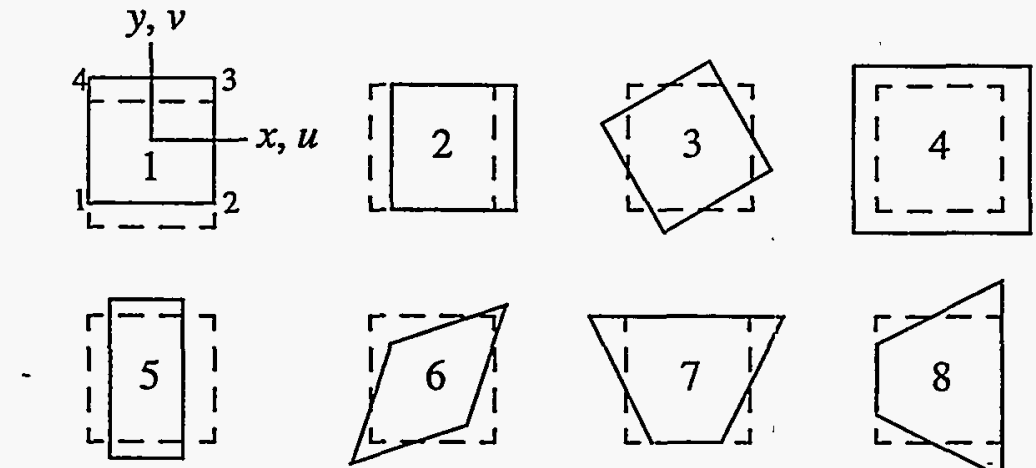

Fig. 15: Independent displacement modes of a quadrilateral element.

The first three displacements are translation and rotation, or rigid-body motion. The next three are considered constant-strain modes, in which the elongation per unit length in the $x$-and $y$-directions are constant. The last two are bending modes, or "hourglass" modes.

Isoparametric coordinates are used to formulate the elements and generate the basis functions for the finite element computation. Isoparametric coordinates for a plane are 
shown in Fig. 16.

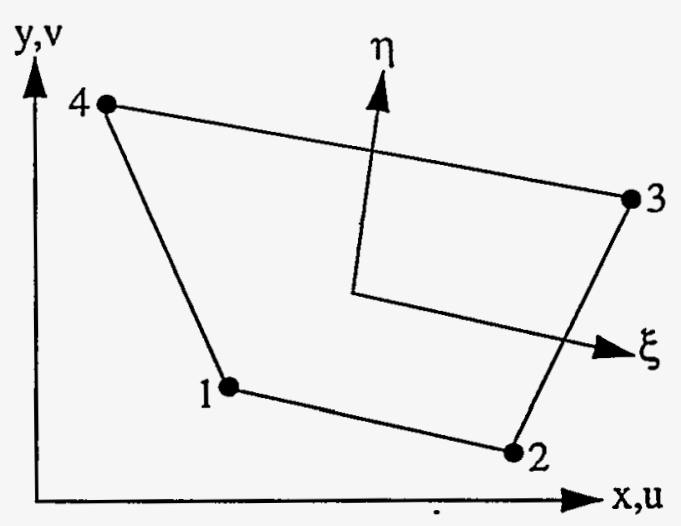

(a)

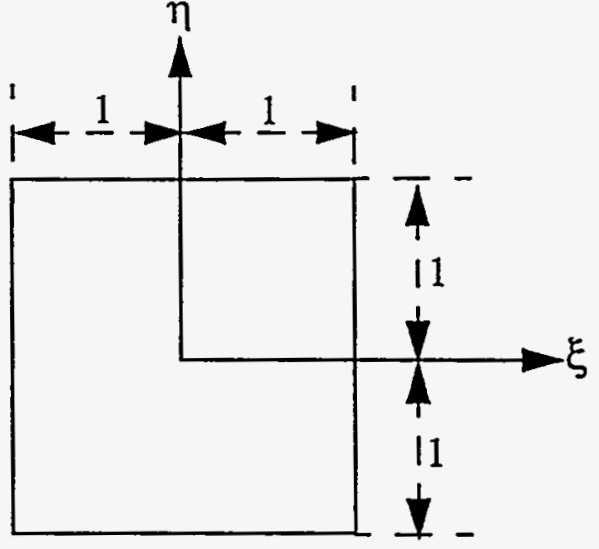

(b)

Fig. 16: (a) Four-noded plane isoparametric element in $\xi \eta$-space.

(b) Plane isoparametric element in $\xi_{\eta}$ space.

The individual shape, or basis functions are defined as

$$
\begin{array}{ll}
N_{1}=\frac{1}{4}(1-\xi)(1-\eta) & N_{3}=\frac{1}{4}(1+\xi)(1+\eta) \\
N_{2}=\frac{1}{4}(1+\xi)(1-\eta) & N_{4}=\frac{1}{4}(1-\xi)(1+\eta)
\end{array}
$$

The coordinates $x$ and $y$ within the element are defined as

$$
x=\sum_{i=1}^{4} N_{i} x_{i} \quad y=\sum_{i=1}^{4} N_{i} y_{i}
$$

and the displacements, $u$ and $v$ within the element are defined as

$$
u=\sum_{i=1}^{4} N_{i} u_{i} \quad v=\sum_{i=1}^{4} N_{i} v_{i}
$$

with the summation over the four nodes defining the quadrilateral. Notice that the displacements $u$ and $v$ are $x$-parallel and $y$-parallel, not $\xi$-parallel and $\eta$-parallel, and the 
shape functions, $N_{i}$ are functions of $\xi$ and $\eta$.

The Jacobian matrix, $\mathbf{J}$, is defined as

$$
\mathbf{J}=\left[\begin{array}{ll}
x_{\prime \xi} & y_{\prime \xi} \\
x_{\prime \eta} & y_{\prime \eta}
\end{array}\right]=\left[\begin{array}{ll}
\sum N_{i, \xi} x_{i} & \sum_{i} N_{i, \xi} y_{i} \\
\sum N_{i, \eta} x_{i} & \sum_{i} N_{i, \eta} y_{i}
\end{array}\right],
$$

where Eq. 103 has been substituted for $x$ and $y$, and the summation over $i$ is implied. The determinant of the Jacobian matrix, $j$, is defined as

$$
j=\operatorname{det}(\mathbf{J})=J_{11} J_{22}-J_{21} J_{12}=x_{\prime \xi} y_{\prime \eta}-x_{\prime \eta} y_{\prime \xi} .
$$

Finally, the inverse of the Jacobian matrix, $\Gamma$, is

$$
\Gamma=\frac{1}{j}\left[\begin{array}{cc}
J_{22} & -J_{12} \\
-J_{21} & J_{11}
\end{array}\right]=\frac{1}{j}\left[\begin{array}{cc}
y_{\prime \eta} & -y_{\prime \xi} \\
-x_{\prime} \eta & x_{\prime \xi}
\end{array}\right]=\left[\begin{array}{ll}
\Gamma_{11} & \Gamma_{12} \\
\Gamma_{21} & \Gamma_{22}
\end{array}\right] .
$$

Using the definition for the inverse, $\Gamma$, the matrix equation for the transformation of variables from $\xi \eta$-space to $x y$-space for the partial derivatives of the displacements is written as

$$
\left[\begin{array}{l}
u_{\prime_{x}} \\
u_{y} \\
v_{\prime_{x}} \\
v_{\prime_{y}}
\end{array}\right]=\left[\begin{array}{cccc}
\Gamma_{11} & \Gamma_{12} & 0 & 0 \\
\Gamma_{21} & \Gamma_{22} & 0 & 0 \\
0 & 0 & \Gamma_{11} & \Gamma_{12} \\
0 & 0 & \Gamma_{21} & \Gamma_{22}
\end{array}\right]\left[\begin{array}{c}
u_{\prime} \\
u_{\prime_{\eta}} \\
v_{\prime_{\xi}} \\
v_{\prime} \eta
\end{array}\right] .
$$

. The partial derivatives of the displacements with respect to $\xi \eta$-space are defined by taking the partiais of Eq. 104 to yield the following matrix equation 


$$
\left[\begin{array}{c}
u_{\prime} \\
u_{\prime} \\
v_{\prime} \\
v_{\prime \eta}
\end{array}\right]=\left[\begin{array}{cccccccc}
N_{1, \xi} & 0 & N_{2, \xi} & 0 & N_{3, \xi} & 0 & N_{4, \xi} & 0 \\
N_{1, \eta} & 0 & N_{2, \eta} & 0 & N_{3, \eta} & 0 & N_{4, \xi} & 0 \\
0 & N_{1, \xi} & 0 & N_{2, \xi} & 0 & N_{3, \xi} & 0 & N_{4, \xi} \\
0 & N_{1, \eta} & 0 & N_{2, \eta} & 0 & N_{3, \eta} & 0 & N_{4, \eta}
\end{array}\right]\left[\begin{array}{c}
u_{1} \\
v_{1} \\
u_{2} \\
v_{2} \\
u_{3} \\
v_{3} \\
u_{4} \\
v_{4}
\end{array}\right]
$$

The strain-displacement relation ${ }^{24}$ for this four-node plane element in two dimensions is given by

$$
\varepsilon=\left[\begin{array}{c}
\varepsilon_{x} \\
\varepsilon_{y} \\
\tau_{x y}
\end{array}\right]=\left[\begin{array}{c}
\frac{\partial u}{\partial x} \\
\frac{\partial v}{\partial y} \\
\frac{\partial u}{\partial y}+\frac{\partial v}{\partial x}
\end{array}\right]=\left[\begin{array}{llll}
1 & 0 & 0 & 0 \\
0 & 0 & 0 & 1 \\
0 & 1 & 1 & 0
\end{array}\right]\left[\begin{array}{l}
u_{\prime} x \\
u_{y} \\
v_{\prime_{x}} \\
v_{y}
\end{array}\right] .
$$

The matrix representation of the strain-displacement relation is

$$
\vec{\varepsilon}=\mathbf{B} \vec{d},
$$

where $\vec{d}$ is the displacements of the plane element

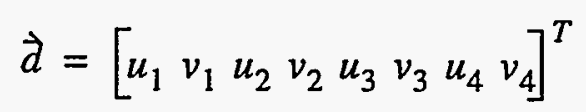

and $B$ is the product of the rectangular matrices in Eq. 108 , Eq. 109 , and Eq. 110 , 


$$
\mathbf{B}=\frac{1}{j}\left[\begin{array}{llll}
1 & 0 & 0 & 0 \\
0 & 0 & 0 & 1 \\
0 & 1 & 1 & 0
\end{array}\right]\left[\begin{array}{cccc}
\Gamma_{11} & \Gamma_{12} & 0 & 0 \\
\Gamma_{21} & \Gamma_{22} & 0 & 0 \\
0 & 0 & \Gamma_{11} & \Gamma_{12} \\
0 & 0 & \Gamma_{21} & \Gamma_{22}
\end{array}\right]\left[\begin{array}{cccccccc}
N_{1, \xi} & 0 & N_{2, \xi} & 0 & N_{3, \xi} & 0 & N_{4, \xi} & 0 \\
N_{1, \eta} & 0 & N_{2, \eta} & 0 & N_{3, \eta} & 0 & N_{4, \xi} & 0 \\
0 & N_{1, \xi} & 0 & N_{2, \xi} & 0 & N_{3, \xi} & 0 & N_{4, \xi} \\
0 & N_{1, \eta} & 0 & N_{2, \eta} & 0 & N_{3, \eta} & 0 & N_{4, \eta}
\end{array}\right]
$$

The plane stress problem can be expressed in terms of an element stiffness matrix, $k$, and the displacement vector, $\vec{d}$ when formulated using finite element analysis. The element stiffness matrix for this problem is defined $a^{6}$

$$
\mathbf{k}=\iint \mathbf{B}^{T} \mathbf{E B} t d x d y=\int_{-1}^{1} \int_{-1}^{1} \mathbf{B}^{T} \mathbf{E B} t j d \xi d \eta,
$$

where $\mathbf{B}$ is defined in Eq. 113, $t$ is the thickness of the element, $\mathbf{E}$ is a metric of material properties, and $j$ is the determinant of the Jacobian, defined in Eq. 106. For this example, let $t=1$, and $\mathbf{E}$ be the identity matrix.

Further expanding the matrix $\mathbf{B}$, and using Eq. 107 for the definition of the inverse of the Jacobian, $\Gamma$, yields the following $3 \times 8$ matrix

$$
\mathbf{B}=\frac{1}{\bar{j}}\left[\begin{array}{cccccccc}
A_{1} & 0 & A_{2} & 0 & A_{3} & 0 & A_{4} & 0 \\
0 & B_{1} & 0 & B_{2} & 0 & B_{3} & 0 & B_{4} \\
B_{1} & A_{1} & B_{2} & A_{2} & B_{3} & A_{3} & B_{4} & A_{4}
\end{array}\right]
$$

where

$$
\begin{aligned}
& A_{i}=y_{\prime \eta} N_{i, \xi}-y_{\prime \xi} N_{i, \eta} \\
& B_{i}=-x_{\prime \eta} N_{i, \xi}+x_{\prime \xi} N_{i, \eta} .
\end{aligned}
$$

Substituting Eq. 115 into Eq. 114 and simplifying allows the element stiffness 
matrix, $k$, to be written as

$$
\mathbf{k}=\int_{-1}^{1} \int_{-1}^{1} \frac{1}{j}\left[\begin{array}{cccc}
A_{1} & 0 & B_{1} \\
0 & B_{1} & A_{1} \\
A_{2} & 0 & B_{2} \\
0 & B_{2} & A_{2} \\
A_{3} & 0 & B_{3} \\
0 & B_{3} & A_{3} \\
A_{4} & 0 & B_{4} \\
0 & B_{4} & A_{4}
\end{array}\right]\left[\begin{array}{cccccccc}
A_{1} & 0 & A_{2} & 0 & A_{3} & 0 & A_{4} & 0 \\
0 & B_{1} & 0 & B_{2} & 0 & B_{3} & 0 & B_{4} \\
B_{1} & A_{1} & B_{2} & A_{2} & B_{3} & A_{3} & B_{4} & A_{4}
\end{array}\right] d \xi d \eta
$$

which, when expanded, yields the following $8 \times 8$ matrix 
$k=\int_{-1}^{1} \int_{-1}^{1} \frac{1}{j}\left[\begin{array}{cccc}A_{1}^{2}+B_{1}^{2} & A_{1} B_{1} & A_{1} A_{2}+B_{1} B_{2} & A_{2} B_{1} \\ A_{1} B_{1} & A_{1}^{2}+B_{1}^{2} & A_{1} B_{2} & A_{1} A_{2}+B_{1} B_{2} \\ A_{1} A_{2}+B_{1} B_{2} & A_{1} B_{2} & A_{2}^{2}+B_{2}^{2} & A_{2} B_{2} \\ A_{2} B_{1} & A_{1} A_{2}+B_{1} B_{2} & A_{2} B_{2} & A_{2}^{2}+B_{2}^{2} \\ A_{1} A_{3}+B_{1} B_{3} & A_{1} B_{3} & A_{2} A_{3}+B_{2} B_{3} & A_{2} B_{3} \\ A_{3} B_{1} & A_{1} A_{3}+B_{1} B_{3} & A_{3} B_{2} & A_{2} A_{3}+B_{2} B_{3} \\ A_{1} A_{4}+B_{1} B_{4} & A_{1} B_{4} & A_{2} A_{4}+B_{2} B_{4} & A_{2} B_{4} \\ A_{4} B_{1} & A_{1} A_{4}+B_{1} B_{4} & A_{4} B_{2} & A_{2} A_{4}+B_{2} B_{4} \\ & & & \\ A_{1} A_{3}+B_{1} B_{3} & A_{3} B_{1} & A_{1} A_{4}+B_{1} B_{4} & A_{4} B_{1} \\ A_{1} B_{3} & A_{1} A_{3}+B_{1} B_{3} & A_{1} B_{4} & A_{1} A_{4}+B_{1} B_{4} \\ A_{2} A_{3}+B_{2} B_{3} & A_{3} B_{2} & A_{2} A_{4}+B_{2} B_{4} & A_{4} B_{2}^{\circ} \\ A_{2} B_{3} & A_{2} A_{3}+B_{2} B_{3} & A_{2} B_{4} & A_{2} A_{4}+B_{2} B_{4} \\ A_{3}^{2}+B_{3}^{2} & A_{3} B_{3} & A_{3} A_{4}+B_{3} B_{4} & A_{4} B_{3} \\ A_{3} B_{3} & A_{3}^{2}+B_{3}^{2} & A_{3} B_{4} & A_{3} A_{4}+B_{3} B_{4} \\ A_{3} A_{4}+B_{3} B_{4} & A_{3} B_{4} & A_{4}^{2}+B_{4}^{2} & A_{4} B_{4} \\ A_{4} B_{3} & A_{3} A_{4}+B_{3} B_{4} & A_{4} B_{4} & A_{4}^{2}+B_{4}^{2}\end{array}\right] d \xi d \eta$

where $A_{i}$ and $B_{i}$ are defined in Eq. 116 .

Assume the integration for $k$, the element stiffness matrix, is to be evaluated over the area of the isoparametric quadraleteral described in Fig. 16. Evaluate this integral using a traditional one-point integration scheme such as the midpoint rule or single point Gaussian quadrature rule. For example, given the following integral

$$
I=\int_{\xi_{1}}^{\xi_{2}} \int_{\eta_{1}}^{\eta_{2}} f(\xi, \eta) d \xi d \eta,
$$

applying the midpoint rule ${ }^{30}$ yields 


$$
I=\left(\xi_{2}-\xi_{1}\right)\left(\eta_{2}-\eta_{1}\right) f\left(\frac{\xi_{2}+\xi_{1}}{2}, \frac{\eta_{2}+\eta_{1}}{2}\right)
$$

Eq. 120 shows that the function, $f(x, h)$, is evaluated at the center of the quadrilateral.

Using the definition in Eq. 120 for the midpoint rule, the element stiffness matrix $k$ can be evaluated. First, the derivatives of the basis functions specified in Eq. 102 are

$$
\begin{aligned}
& \frac{\partial}{\partial \xi} N_{1}(0,0)=N_{1, \xi}=-\frac{1}{4}(1-\eta)=-\frac{1}{4} \\
& \frac{\partial}{\partial \xi} N_{2}(0,0)=N_{2, \xi}=\frac{1}{4}(1-\eta)=\frac{1}{4} \\
& \frac{\partial}{\partial \xi} N_{3}(0,0)=N_{3, \xi}=\frac{1}{4}(1+\eta)=\frac{1}{4} \\
& \frac{\partial}{\partial \xi} N_{4}(0,0)=N_{4, \xi}=-\frac{1}{4}(1+\eta)=-\frac{1}{4} \\
& \frac{\partial}{\partial \eta} N_{1}(0,0)=N_{1, \eta}=-\frac{1}{4}(1-\xi)=-\frac{1}{4} \\
& \frac{\partial}{\partial \eta} N_{2}(0,0)=N_{2, \eta}=-\frac{1}{4}(1+\xi)=-\frac{1}{4} \\
& \frac{\partial}{\partial \eta} N_{3}(0,0)=N_{3, \eta}=\frac{1}{4}(1+\xi)=\frac{1}{4} \\
& \frac{\partial}{\partial \eta} N_{4}(0,0)=N_{4, \eta}=\frac{1}{4}(1-\xi)=\frac{1}{4}
\end{aligned}
$$

Applying the midpoint rule in Eq. 120 to $k$ specified by Eq. 118 , with $\Phi(\xi$, $\eta)$ representing the $8 \times 8$ matrix in that equation, yields

$$
\mathbf{k}=\int_{-1}^{1} \int_{-1}^{1} \Phi(\xi, \eta) d \xi d \eta=2 \int_{-1}^{1} \Phi(\xi, 0) d \xi d \eta=4(\Phi(0,0))
$$

Finally, evaluating $k$ results in the following $8 \times 8$ matrix 


$$
\mathbf{k}=\frac{1}{j}\left[\begin{array}{llllllll}
k_{11} & k_{12} & k_{13} & k_{14} & k_{15} & k_{16} & k_{17} & k_{18} \\
k_{21} & k_{22} & k_{23} & k_{24} & k_{25} & k_{26} & k_{27} & k_{28} \\
k_{31} & k_{32} & k_{33} & k_{34} & k_{35} & k_{36} & k_{37} & k_{38} \\
k_{41} & k_{42} & k_{43} & k_{44} & k_{45} & k_{46} & k_{47} & k_{48} \\
k_{51} & k_{52} & k_{53} & k_{54} & k_{55} & k_{56} & k_{57} & k_{58} \\
k_{61} & k_{62} & k_{63} & k_{64} & k_{65} & k_{66} & k_{67} & k_{68} \\
k_{71} & k_{72} & k_{73} & k_{74} & k_{75} & k_{76} & k_{77} & k_{78} \\
k_{81} & k_{82} & k_{83} & k_{84} & k_{85} & k_{86} & k_{87} & k_{88}
\end{array}\right]
$$

where

$$
\begin{aligned}
& k_{11}=k_{22}=\left(-y_{\prime \eta}+y_{\prime \xi}\right)^{2}+\left(x_{\prime \eta}-x_{\prime \xi}\right)^{2} \\
& k_{33}=k_{44}=\left(y_{\prime \eta}+y_{\prime \xi}\right)^{2}+\left(-x_{\prime}-x_{\prime \xi}\right)^{2} \\
& k_{55}=k_{66}=\left(y_{\prime \eta}-y_{\prime \xi}\right)^{2}+\left(-x_{\prime \eta}+x_{\prime \xi}\right)^{2} \\
& k_{77}=k_{88}=\left(-y_{\prime \eta}-y_{\prime \xi}\right)^{2}+\left(x_{\prime \eta}+x_{\prime \xi}\right)^{2} \\
& k_{12}=k_{21}=\left(-y_{\prime \eta}+y_{\prime \xi}\right)\left(x_{\prime}-x_{\prime \xi}\right) \\
& k_{13}=k_{31}=k_{24}=k_{42}=\left(-y_{\prime}+y_{\prime \xi}\right)\left(y_{\prime \eta}+y_{\prime \xi}\right)+\left(x_{\prime \eta}-x_{\prime \xi}\right)\left(-x_{\prime \eta}-x_{\prime \xi}\right) \\
& k_{14}=k_{41}=\left(y_{\prime \eta}+y_{\prime \xi}\right)\left(x_{\prime \eta}-x_{\prime \xi}\right) \\
& k_{15}=k_{51}=k_{26}=k_{62}=\left(-y_{\prime}+y_{\prime \xi}\right)\left(y_{\prime \eta}-y_{\prime \xi}\right)+\left(x_{\prime \eta}-x_{\prime \xi}\right)\left(-x_{\prime}+x_{\prime \xi}\right) \\
& k_{16}=k_{61}=\left(y_{\prime \eta}-y_{\prime \xi}\right)\left(x_{\prime \eta}-x_{\prime \xi}\right) \\
& k_{17}=k_{71}=k_{28}=k_{82}=\left(-y_{\prime \prime}+y_{\prime \xi}\right)\left(-y_{\prime \eta}-y_{\prime \xi}\right)+\left(x_{\prime \eta}-x_{\prime \xi}\right)\left(x_{\prime \eta}+x_{\prime \xi}\right) \\
& k_{18}=k_{81}=\left(-y_{\prime \eta}-y_{\prime \xi}\right)\left(x_{\prime \eta}-x_{\prime \xi}\right) \\
& k_{23}=k_{32}=\left(-y_{\prime \eta}+y_{\prime \xi}\right)\left(-x_{\prime}-x_{\prime \xi}\right) \\
& k_{25}=k_{52}=\left(-y_{\prime \eta}+y_{\prime \xi}\right)\left(-x_{\prime \eta}+x_{\prime \xi}\right) \\
& k_{27}=k_{72}=\left(-y_{\prime \eta}+y_{\prime \xi}\right)\left(x_{\prime \eta}+x_{\prime \xi}\right)
\end{aligned}
$$




$$
\begin{aligned}
& k_{34}=k_{43}=\left(y_{\prime \eta}+y_{\prime \xi}\right)\left(-x_{\prime \eta}-x_{\prime \xi}\right) \\
& k_{35}=k_{53}=k_{46}=k_{64}=\left(y_{\prime \eta}+y_{\prime \xi}\right)\left(y_{\prime \eta}-y_{\prime \xi}\right)+\left(-x_{\prime \eta}-x_{\prime \xi}\right)\left(-x_{\prime \eta}+x_{\prime \xi}\right) \\
& k_{36}=k_{63}=\left(y_{\prime \eta}-y_{\prime \xi}\right)\left(-x_{\prime \eta}-x_{\prime \xi}\right) \\
& k_{37}=k_{73}=k_{48}=k_{84}=\left(y_{\prime \eta}+y_{\prime \xi}\right)\left(-y_{\prime \eta}-y_{\prime \xi}\right)+\left(-x_{\prime \eta}-x_{\prime \xi}\right)\left(x_{\prime \eta}+x_{\prime \xi}\right) \\
& k_{38}=k_{83}=\left(-y_{\prime \eta}-y_{\prime \xi}\right)\left(-x_{\prime \eta}-x_{\prime \xi}\right) \\
& k_{45}=k_{54}=\left(y_{\prime \eta}+y_{\prime \xi}\right)\left(-x_{\prime \eta}+x_{\prime \xi}\right) \\
& k_{47}=k_{74}=\left(y_{\prime \eta}+y_{\prime \xi}\right)\left(x_{\prime}+x_{\prime \xi}\right) \\
& k_{56}=k_{65}=\left(y_{\prime \eta}-y_{\prime \xi}\right)\left(-x_{\prime \eta}+x_{\prime \xi}\right) \\
& k_{57}=k_{75}=k_{68}=k_{86}=\left(y_{\prime \eta}-y_{\prime \xi}\right)\left(-y_{\prime \eta}-y_{\prime \xi}\right)+\left(-x_{\prime}+x_{\prime \xi}\right)\left(x_{\prime \eta}+x_{\prime \xi}\right) \\
& k_{58}=k_{85}=\left(-y_{\prime \eta}-y_{\prime \xi}\right)\left(-x_{\prime \eta}+x_{\prime \xi}\right) \\
& k_{67}=k_{76}=\left(y_{\prime \eta}-y_{\prime \xi}\right)\left(x_{\prime \eta}+x_{\prime \xi}\right) \\
& k_{78}=k_{87}=\left(-y_{\prime \eta}-y_{\prime \xi}\right)\left(x_{\prime \eta}+x_{\prime \xi}\right)
\end{aligned}
$$

Now that the element stiffness matrix, $k$, has been defined, the eigenvalues of this matrix can be evaluated. Using the form of the stiffness matrix equation defined in Eq. 100 , the eigenproblem ${ }^{14}$ for this matrix is defined as

$$
\mathbf{k} \vec{d}=\lambda \vec{d} \text { or }(\mathbf{k}-\lambda \mathbf{I}) \vec{d}=\mathbf{0} .
$$

Eigenvalues $\lambda_{2}$ are called the eigenvalues of $k$, and there are as many $\lambda_{2}$ as there are entries in $\vec{a}$. For this two dimensional case, then, there are $8 \lambda_{\imath}$. Not all $\lambda_{1}$ need be unique, and for each $\lambda_{\imath}$ there corresponds an eigenvector $\vec{d}_{i}$, where $\vec{d}_{i}$ is the normalized displacement vector such that

$$
\vec{d}_{i}^{T} \vec{d}_{i}=1
$$

Multiplying Eq. 125 by $\vec{d}_{i}^{T}$ yields

$$
\vec{a}_{i}^{T} \mathbf{k} \vec{d}_{i}=\lambda_{i}
$$


For rigid body motion ${ }^{6}$, shown in Fig. 15 as the first three independent displacement modes of a quadrilateral element, there should be three $\lambda_{i}=0$. If any hourglass or zero-energy modes are present in the stiffness matrix, there will be more $\lambda_{l}$ that equal zero.

Eq. 127 can be applied to the element stiffness matrix, $k$, defined by Eq. 123 and the displacement vector, $\vec{d}$, defined in Eq. 112 to determine if any hourglass modes are present for this particular $k$. For the first rigid body mode defined in Fig. 15 , the displacement vector and normalized displacement vector are

$$
\vec{a}=\left[\begin{array}{l}
u_{1} \\
v_{1} \\
u_{2} \\
v_{2} \\
u_{3} \\
v_{3} \\
u_{4} \\
v_{4}
\end{array}\right]=\left[\begin{array}{l}
0 \\
1 \\
0 \\
1 \\
0 \\
1 \\
0 \\
1
\end{array}\right] \text { and } \overrightarrow{d_{1}}=\frac{1}{2}\left[\begin{array}{l}
0 \\
1 \\
0 \\
0 \\
1 \\
0 \\
1
\end{array}\right] .
$$

Substituting the above into Eq. 127 yields

$$
\lambda_{1}=\vec{a}_{1}^{T} \mathbf{k} \vec{a}_{1}=\frac{1}{4}\left[\begin{array}{llllllll}
0 & 1 & 0 & 1 & 0 & 1 & 0 & 1
\end{array}\right] \mathbf{k}\left[\begin{array}{l}
0 \\
1 \\
0 \\
1 \\
0 \\
1 \\
0 \\
1
\end{array}\right]
$$


or,

$$
\begin{gathered}
\lambda_{1}=\frac{1}{4 j}\left(k_{22}+k_{24}+k_{26}+k_{28}+k_{42}+k_{44}+k_{46}+k_{48}+\right. \\
\left.k_{62}+k_{64}+k_{66}+k_{68}+k_{82}+k_{84}+k_{86}+k_{88}\right)
\end{gathered}
$$

Substituting Eq. 124 into the above equation yields

$$
\lambda_{1}=0 \text {, }
$$

which is what is expected for rigid body motion. Applying the same technique to the second and third independent displacement modes defined in Fig. 15 yield zero as the $\lambda_{1}$.

The displacement vector for the seventh mode, an hourglass mode, is defined as

$$
\overrightarrow{d_{7}}=\left[\begin{array}{l}
u_{1} \\
v_{1} \\
u_{2} \\
v_{2} \\
u_{3} \\
v_{3} \\
u_{4} \\
v_{4}
\end{array}\right]=\left[\begin{array}{c}
1 \\
0 \\
-1 \\
0 \\
1 \\
0 \\
-1 \\
0
\end{array}\right]
$$

Substituting the above into Eq. 127 gives

$$
\begin{array}{r}
\lambda_{7}=\frac{1}{4 . i}\left(k_{11}-k_{13}+k_{15}-k_{17}-k_{31}+k_{33}-k_{35}+k_{37}+\right. \\
\left.k_{51}-k_{53}+k_{55}-k_{57}-k_{71}+k_{73}-k_{75}+k_{77}\right)
\end{array}
$$

and further substituting Eq. 124 into the above equation yields

$$
\lambda_{7}=0 .
$$

Applying the above approach to displacement mode 8, another hourglass mode shown in 
Fig. 15 results in

$$
\lambda_{8}=0
$$

The element stiffness matrix, then, for this two dimensional problem has zero-energy or hourglass modes, which will generate instabilities in the solution if these modes are not controlled.

Various control methods have been proposed in the literature. The simplest approach involves adding a "stabilization matrix" 6 of the form

$$
\mathbf{k}_{7}=\left(a_{7} \vec{a}_{7}\right)\left(a_{7} \vec{a}_{7}\right)^{T}
$$

to the element stiffness matrix, $k$, where $\alpha_{7}$ is some constant. More complex approaches are defined by Kosloff and Frazier ${ }^{20}$ and Flanagan and Bielytschko ${ }^{11}$. The latter approach is now a standard technique for isolating and controlling hourglass modes.

The term hourglassing has now been defined, and a simple two-dimensional example has been used to illustrate the origins of the hourglass terms in the solution. Next, the method used for removing, or damping the hourglass phenomena from the diffusive mesh relaxer is described. 


\section{Hourglass Control in the Diffusive Mesh Relaxer}

The technique used for isolating and controlling the hourglass modes found in the diffusive mesh relaxer is that presented by Dr. Robert E. Tipton ${ }^{37}$. He applies an hourglass isolation and controlling technique to an Equipotential mesh relaxer, and thus it seemed obvious to apply this method to the diffusive mesh relaxer. This technique proved acceptable in controlling the hourglass modes seen in the diffusive relaxer. First, the diffusive mesh relaxer is represented from a variational principle, and the resulting functional is viewed as a sum over the individual elements. The element stiffness matrix for this functional is then analyzed for hourglass modes, and finally, the matrix is manipulated to control these unphysical modes.

It is instructive to first define several terms for the three dimensional element, the hexahedron. Fig. 17 shows the 24 independent displacement modes $^{11}$, eight for each direction, with the index $i$ representing the three directions. Mode A accounts for rigid body translation, $B_{1 i}$ and $B_{2 i}$ represent constant-strain modes and $B_{3 i}$ is rigid body rotation. The last four independent displacements, $C_{\alpha i}$, where $\alpha$ has a range of four, give rise 
to the bending or hourglass modes.
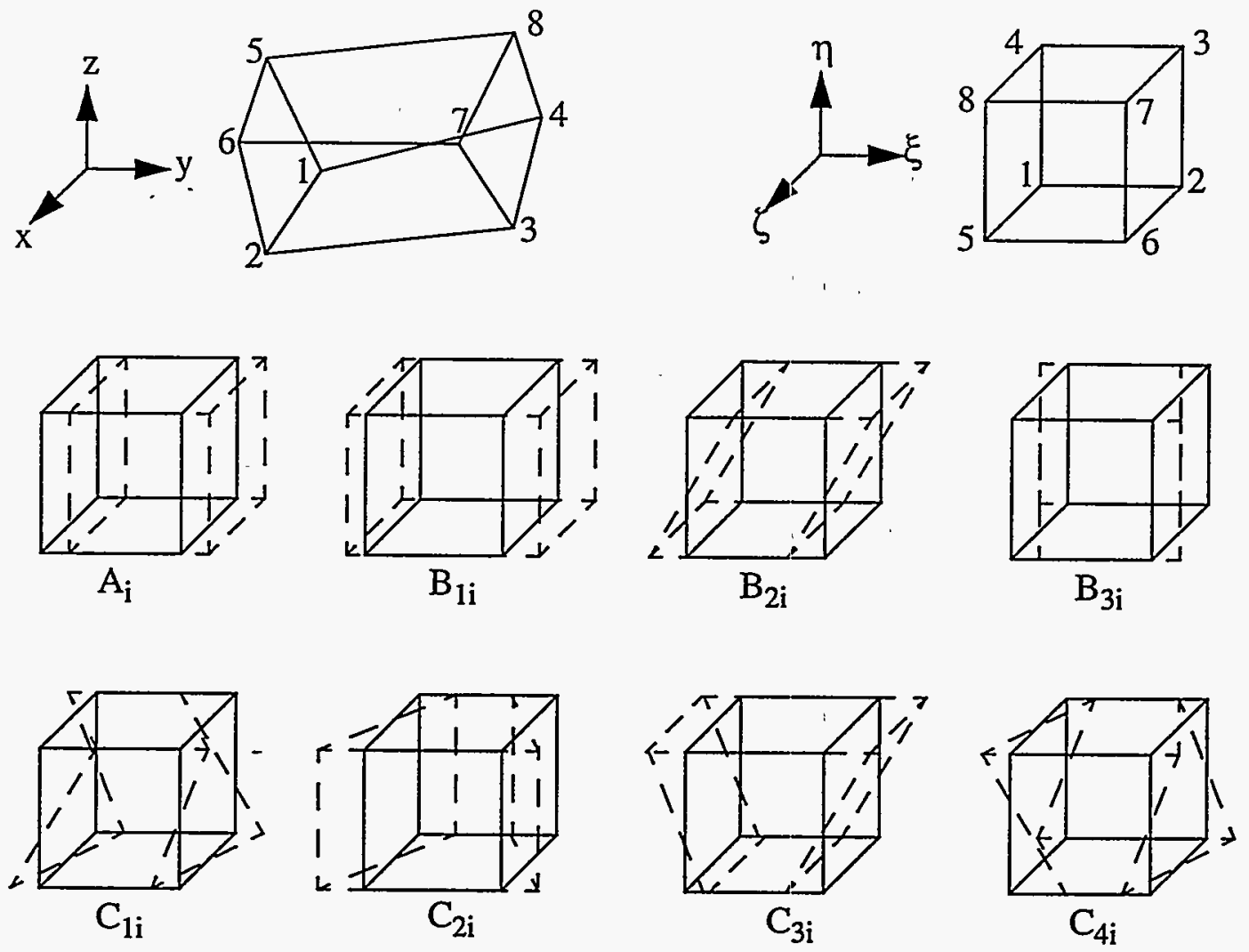

Fig. 17: Hexahedron and its displacement modes; hourglass modes $\mathrm{C}_{\alpha i}$ -

Several displacement vectors depicting the various independent modes in Fig. 17 are shown below.

$$
\overrightarrow{d_{A}}=\left[\begin{array}{l}
u_{1} \\
u_{2} \\
u_{3} \\
u_{4} \\
u_{5} \\
u_{6} \\
u_{7} \\
u_{8}
\end{array}\right]=\left[\begin{array}{l}
1 \\
1 \\
1 \\
1 \\
1 \\
1 \\
1 \\
1
\end{array}\right] \text { for the first rigid body mode, } \mathrm{A}
$$




$$
\overrightarrow{d_{B_{2}}}=\left[\begin{array}{l}
u_{1} \\
u_{2} \\
u_{3} \\
u_{4} \\
u_{5} \\
u_{6} \\
u_{7} \\
u_{8}
\end{array}\right]=\left[\begin{array}{c}
-1 \\
-1 \\
1 \\
1 \\
-1 \\
-1 \\
1 \\
1
\end{array}\right] \text { for the constant-strain mode, } B_{2}, \text { and }
$$

$$
\overrightarrow{d_{C_{3}}}=\left[\begin{array}{l}
u_{1} \\
u_{2} \\
u_{3} \\
u_{4} \\
u_{5} \\
u_{6} \\
u_{7} \\
u_{8}
\end{array}\right]=\left[\begin{array}{c}
1 \\
-1 \\
1 \\
-1 \\
1 \\
-1 \\
1 \\
-1
\end{array}\right] \text { for the hourglass mode, } C_{3}
$$

The first step towards isolating the hourglass modes is to determine the element stiffness matrix, and this step begins with a look at the diffusive mesh relaxer from a variational principle. Let

$$
I=\frac{1}{2} \iiint W|\nabla \xi|^{2} d x^{3}
$$

where

$$
\vec{x}=\left[\begin{array}{l}
x \\
y \\
z
\end{array}\right], W=\left[\begin{array}{l}
W_{1}(x, y, z) \\
W_{2}(x, y, z) \\
W_{3}(x, y, z)
\end{array}\right] \text { and } \vec{\xi}=\left[\begin{array}{l}
\xi(x, y, z) \\
\eta(x, y, z) \\
\zeta(x, y, z)
\end{array}\right]
$$

and the existence of a functional per direction is again implied for simplicity, as in Chap- 
ter 3 .

The variational principle ${ }^{2}$ states that if $\xi(x)$ produces an extremum in $\mathrm{I}$, then

$$
\nabla \bullet(W \nabla \xi)=0
$$

which is the governing equation for the diffusive mesh relaxer. Remember that for finite element formulization, a function can be represented as an expansion of the shape, or basis functions `

$$
\xi=f=\sum_{\alpha} f_{\alpha} N_{\alpha}
$$

where $f_{\alpha}$ is the value of $\xi$ at node $\alpha$, and $N_{\alpha}$ is the basis function at that node. Substituting Eq. 143 into Eq. 140 gives

$$
I=\frac{1}{2} \sum_{\alpha \beta} f_{\alpha} f_{\beta} \iiint W \nabla N_{\alpha} \cdot \nabla N_{\beta} d x^{3}=\frac{1}{2} \sum_{\alpha \beta} f_{\alpha} M_{\alpha \beta} f_{\beta}
$$

where

$$
M_{\alpha \beta}=\iiint W \nabla N_{\alpha} \bullet \nabla N_{\beta} d x^{3}
$$

Recall that for I to be an extremum,

$$
\frac{\partial I}{\partial f_{\alpha}}=\sum_{\alpha \beta} M_{\alpha \beta} f_{\beta}=M_{\alpha \alpha} f_{\alpha}+\sum_{\beta \neq \alpha} M_{\alpha \beta} f_{\beta}=0
$$

which is identical to the diffusive mesh relaxation algorithm specified by Eq. 63 .

The next step necessary for determining the element 'stiffness matrix is to transform Eq. 144 from physical coordinates to logical, or parametric coordinates. Substituting Eq. 65 - Eq. 67 and Eq. 72 into Eq. 144 , and remembering Eq. 143 , gives 


$$
I=\frac{1}{2} \sum_{\alpha \beta} f_{\alpha} f_{\beta} \iiint w \sum_{i, j=1}^{3} a_{i j}\left(\frac{\partial N_{\alpha}}{\partial \xi_{i}}\right) \cdot\left(\frac{\partial N_{\beta}}{\partial \xi_{j}}\right) d \xi^{3}=\frac{1}{2} \iiint w \sum_{i, j=1}^{3} a_{i j}\left(\frac{\partial f}{\partial \xi_{i}}\right) \bullet\left(\frac{\partial f}{\partial \xi_{j}}\right) d \xi^{3}
$$

The elemental contribution, then, to the total variational integral, $I$, is

$$
I_{e l}^{-}=\frac{1}{2} \sum_{\alpha \beta} f_{\alpha} M_{\alpha \beta}^{e l} f_{-\beta}=\frac{1}{2}\left[\iiint w \sum_{i, j=1}^{3} a_{i j}\left(\frac{\partial f}{\partial \xi_{i}}\right) \bullet\left(\frac{\partial f}{\partial \xi_{j}}\right) d \xi^{3}\right]_{e l}
$$

Expanding $I_{e l}$ in Eq. 148 gives

$$
\begin{aligned}
I_{e l}=\frac{1}{2} \int_{-\frac{1}{2}}^{\frac{1}{2}} \int_{-\frac{1}{2}}^{\frac{1}{2}} \int_{-\frac{1}{2}}^{\frac{1}{2}} w_{e l} & {\left[a_{11}\left(\frac{\partial f}{\partial \xi}\right)\left(\frac{\partial f}{\partial \xi}\right)+a_{12}\left(\frac{\partial f}{\partial \xi}\right)\left(\frac{\partial f}{\partial \eta}\right)+a_{13}\left(\frac{\partial f}{\partial \xi}\right)\left(\frac{\partial f}{\partial \zeta}\right)+\right.} \\
& a_{21}\left(\frac{\partial f}{\partial \eta}\right)\left(\frac{\partial f}{\partial \xi}\right)+a_{22}\left(\frac{\partial f}{\partial \eta}\right)\left(\frac{\partial f}{\partial \eta}\right)+a_{23}\left(\frac{\partial f}{\partial \eta}\right)\left(\frac{\partial f}{\partial \zeta}\right)+ \\
& \left.a_{31}\left(\frac{\partial f}{\partial \zeta}\right)\left(\frac{\partial f}{\partial \xi}\right)+a_{32}\left(\frac{\partial f}{\partial \zeta}\right)\left(\frac{\partial f}{\partial \eta}\right)+a_{33}\left(\frac{\partial f}{\partial \zeta}\right)\left(\frac{\partial f}{\partial \zeta}\right)\right] d \xi^{3}
\end{aligned}
$$

Now that the elemental variational integral has been defined, it is appropriate to analyze this functional for hourglass modes. Define the logical element as a unit cube with 
the center of the logical axis at $(0,0,0)$, and the nodes numbered as shown in Fig. 18 .

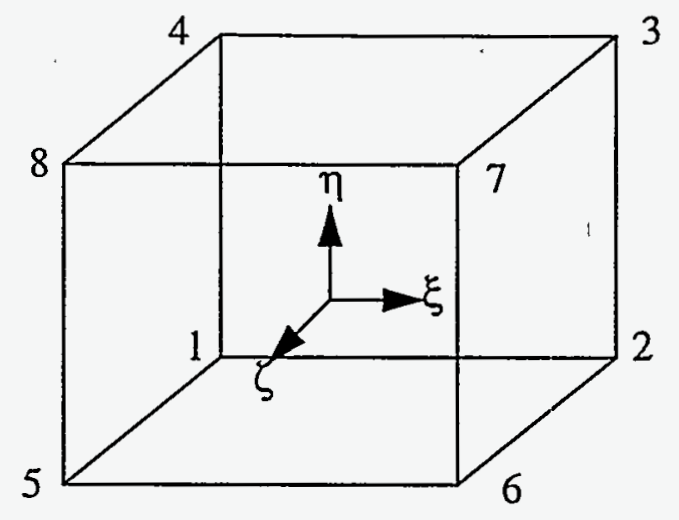

Fig. 18: Logical Unit Cube with Node Numbers

Given the unit cube in Fig. 18 , let the parametric coordinates be defined as

$$
\begin{aligned}
& -\frac{1}{2} \leq \xi \leq \frac{1}{2} \\
& -\frac{1}{2} \leq \eta \leq \frac{1}{2} \\
& -\frac{1}{2} \leq \zeta \leq \frac{1}{2}
\end{aligned}
$$

and the corresponding basis or shape functions for each node in Fig. 18 is 


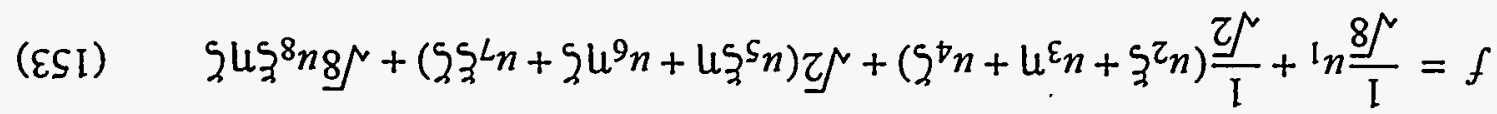

u!̣ s][nsว ถిน!̣\}

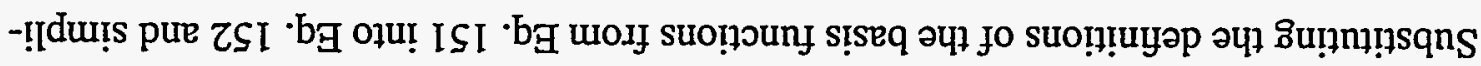
$(\mathrm{ZSI})^{8} N^{8} f+{ }^{L} N^{L} f+{ }^{9} N^{9} f+{ }^{\varsigma} N^{\varsigma} f+{ }^{\dagger} N^{\dagger} f+{ }^{\varepsilon} N^{\varepsilon} f+{ }^{2} N^{\tau} f+{ }^{\mathrm{I}} N^{\mathrm{I}} f={ }^{\infty} N^{\infty} f{ }_{8}^{\mathrm{I}=\infty}=f$

suo!̣ouny

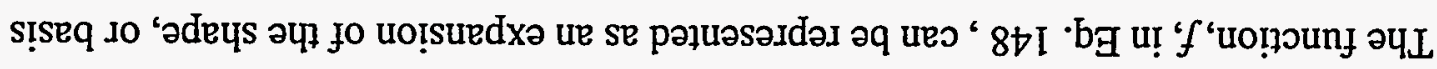
(ISI)

$$
\begin{aligned}
& \left(\xi+\frac{\tau}{l}\right)\left(u+\frac{\tau}{l}\right)\left(\xi-\frac{\tau}{l}\right)={ }^{8} N \\
& \left(\xi+\frac{\tau}{l}\right)\left(u+\frac{\tau}{l}\right)\left(\xi+\frac{\tau}{l}\right)={ }^{L} N \\
& \left(\xi+\frac{\tau}{l}\right)\left(u-\frac{\tau}{l}\right)\left(\xi+\frac{\tau}{l}\right)={ }^{9} N \\
& \left(\xi+\frac{\tau}{l}\right)\left(u-\frac{\tau}{l}\right)\left(\xi-\frac{\tau}{l}\right)={ }^{s} N \\
& \left(\xi-\frac{\tau}{l}\right)\left(u+\frac{\tau}{l}\right)\left(\xi-\frac{\tau}{l}\right)={ }^{t} N \\
& \left(\xi-\frac{\tau}{l}\right)\left(u+\frac{\tau}{l}\right)\left(\xi+\frac{\tau}{l}\right)={ }^{\varepsilon} N \\
& \left(\xi-\frac{\tau}{l}\right)\left(u-\frac{\tau}{l}\right)\left(\xi+\frac{\tau}{l}\right)={ }^{\tau} N \\
& \left(\xi-\frac{\tau}{l}\right)\left(u-\frac{\tau}{l}\right)\left(\xi-\frac{\tau}{l}\right)={ }^{1} N
\end{aligned}
$$




$$
\begin{aligned}
& u_{1}=\frac{1}{\sqrt{8}}\left(f_{1}+f_{2}+f_{3}+f_{4}+f_{5}+f_{6}+f_{7}+f_{8}\right) \\
& u_{2}=\frac{1}{\sqrt{8}}\left(-f_{1}+f_{2}+f_{3}-f_{4}-f_{5}+f_{6}+f_{7}-f_{8}\right) \\
& u_{3}=\frac{1}{\sqrt{8}}\left(-f_{1}-f_{2}+f_{3}+f_{4}-f_{5}-f_{6}+f_{7}+f_{8}\right) \\
& u_{4}=\frac{1}{\sqrt{8}}\left(-f_{1}-f_{2}-f_{3}-f_{4}+f_{5}+f_{6}+f_{7}+f_{8}\right) \\
& u_{5}=\frac{1}{\sqrt{8}}\left(f_{1}-f_{2}+f_{3}-f_{4}+f_{5}-f_{6}+f_{7}-f_{8}\right) \\
& u_{6}=\frac{1}{\sqrt{8}}\left(f_{1}+f_{2}-f_{3}-f_{4}-f_{5}-f_{6}+f_{7}+f_{8}\right) \\
& u_{7}=\frac{1}{\sqrt{8}}\left(f_{1}-f_{2}-f_{3}+f_{4}-f_{5}+f_{6}+f_{7}-f_{8}\right) \\
& u_{8}=\frac{1}{\sqrt{8}}\left(-f_{1}+f_{2}-f_{3}+f_{4}+f_{5}-f_{6}+f_{7}-f_{8}\right)
\end{aligned}
$$

or in vector notation

$$
\vec{u}=\frac{1}{\sqrt{8}} T \cdot \vec{f}=\frac{1}{\sqrt{8}}\left[\begin{array}{cccccccc}
1 & 1 & 1 & 1 & 1 & 1 & 1 & 1 \\
-1 & 1 & 1 & -1 & -1 & 1 & 1 & -1 \\
-1 & -1 & 1 & 1 & -1 & -1 & 1 & 1 \\
-1 & -1 & -1 & -1 & 1 & 1 & 1 & 1 \\
1 & -1 & 1 & -1 & 1 & -1 & 1 & -1 \\
1 & 1 & -1 & -1 & -1 & -1 & 1 & 1 \\
1 & -1 & -1 & 1 & -1 & 1 & 1 & -1 \\
-1 & 1 & -1 & 1 & 1 & -1 & 1 & -1
\end{array}\right]\left[\begin{array}{c}
f_{1} \\
f_{2} \\
f_{3} \\
f_{4} \\
f_{5} \\
f_{6} \\
f_{7} \\
f_{8}
\end{array}\right] .
$$

It is worthwhile to compare the above definitions of $u$ in Eq. 154 with the displacement vectors generated by the independent displacement modes, seen in Fig. 17. $u_{l}$ can be represented as 
$u_{1}=\frac{1}{\sqrt{8}} \vec{a}_{1}^{T} \cdot \vec{f}=\frac{1}{\sqrt{8}}\left[\begin{array}{llllllll}1 & 1 & 1 & 1 & 1 & 1 & 1 & 1\end{array}\right] \cdot\left[\begin{array}{c}f_{1} \\ f_{2} \\ f_{3} \\ f_{4} \\ f_{5} \\ f_{6} \\ f_{7} \\ f_{8}\end{array}\right]$

where $\vec{d}_{1}=\vec{a}_{A}$ for the first rigid body mode, A. $u_{3}$ can be represented as

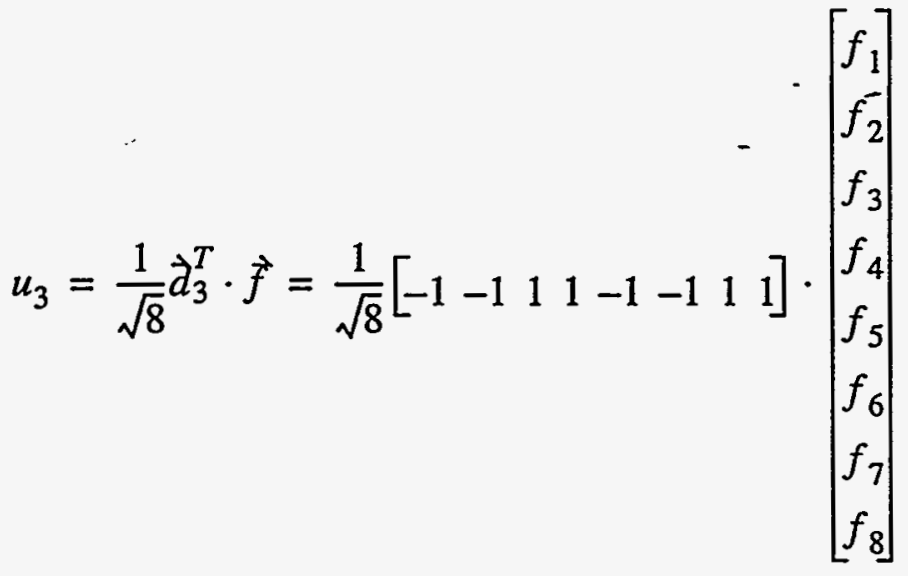

where $\vec{d}_{3}=\vec{d}_{B_{2}}$ for the constant-strain mode, $B_{2} \cdot u_{5}$ can be represented as

$$
u_{5}=\frac{1}{\sqrt{8}} \vec{a}_{5}^{T} \cdot \vec{f}=\frac{1}{\sqrt{8}}\left[\begin{array}{llllllll}
1 & -1 & 1 & -1 & 1 & -1 & 1 & -1
\end{array}\right] \cdot\left[\begin{array}{l}
f_{1} \\
f_{2} \\
f_{3} \\
f_{4} \\
f_{5} \\
f_{6} \\
f_{7} \\
f_{8}
\end{array}\right]
$$


where $\vec{d}_{5}=\vec{a}_{C_{3}}$ for the hourglass mode, $C_{3}$. Similarly, relations between the remaining $u$ 's in Eq. 154 and the displacement vectors associated with the independent displacement modes can be expressed. Thus, $u_{1}-u_{4}$ represent the physical modes and $u_{5}-u_{8}$ represent the unphysical, hourglass modes.

Applying the midpoint rule described by Eq. 120 to Eq. 149 , and substituting in the expansion for function $f$ defined in Eq. 153 results in

$$
I_{e l}=\frac{1}{8} w_{e l}\left\{a_{11} u_{2}^{2}+a_{22} u_{3}^{2}+a_{33} u_{4}^{2}+2 a_{12} u_{2} u_{3}+2 a_{23} u_{3} u_{4}+2 a_{13} u_{2} u_{4}\right\}
$$

since $\xi=0, \eta=0, \zeta=0$ at the midpoint of the element. Restructuring $I_{e l}$ from Eq. 159 into a matrix equation gives

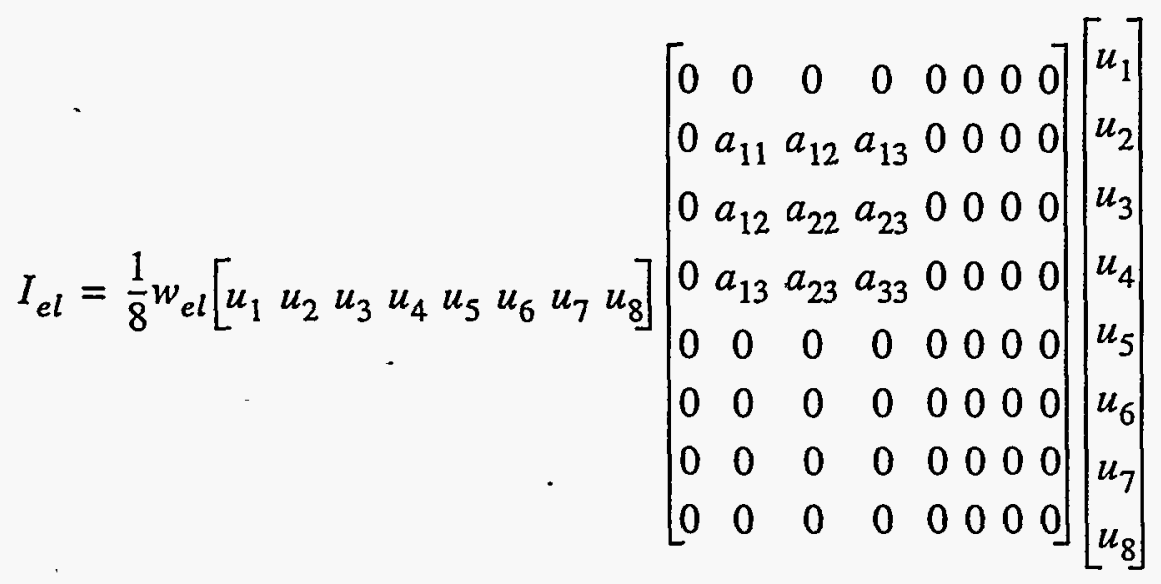

or

$$
I_{e l}=\frac{1}{8} w_{e l} \vec{u}^{T} \cdot M_{e l} \cdot \grave{u}
$$

where 


$$
M_{c l}=\left[\begin{array}{cccccccc}
0 & 0 & 0 & 0 & 0 & 0 & 0 & 0 \\
0 & a_{11} & a_{12} & a_{13} & 0 & 0 & 0 & 0 \\
0 & a_{12} & a_{22} & a_{23} & 0 & 0 & 0 & 0 \\
0 & a_{13} & a_{23} & a_{33} & 0 & 0 & 0 & 0 \\
0 & 0 & 0 & 0 & 0 & 0 & 0 & 0 \\
0 & 0 & 0 & 0 & 0 & 0 & 0 & 0 \\
0 & 0 & 0 & 0 & 0 & 0 & 0 & 0 \\
0 & 0 & 0 & 0 & 0 & 0 & 0 & 0
\end{array}\right]=\left[\begin{array}{ll}
A & B \\
B & C
\end{array}\right]
$$

and

$$
A=\left[\begin{array}{cccc}
0 & 0 & 0 & 0 \\
0 & a_{11} & a_{12} & a_{13} \\
0 & a_{12} & a_{22} & a_{23} \\
0 & a_{13} & a_{23} & a_{33}
\end{array}\right] \text { and } B=C=0
$$

Matrix $M_{e l}$ specified in Eq. 162 is the element stiffness matrix for the diffusive mesh relaxer. Analyzing the eigenvalues of this matrix, using Eq. 125 , results in three nonzero and five zero eigenvalues. One of the zero eigenvalues is for the rigid body mode, $A$, from Fig. 17 , while the remaining four zero eigenvalues represent the four unphysical, hourglass modes, $C_{\alpha}$. The block matrix form of $\mathrm{M}_{e l}$ breaks down into three $4 \times 4$ matrices, $\mathbf{A}, \mathbf{B}$ and $\mathbf{C}$, where $\mathbf{A}$ represents the interactions between the four physical modes, $\mathbf{B}$ represents the interactions among the physical and unphysical modes, and $\mathbf{C}$ rẹpresents the interactions between the unphysical modes.

The element stiffness matrix for the diffusive mesh relaxer has now been analyzed, and hourglass modes have been found to exist. The final step is to manipulate the stiffness matrix, $\mathbf{M}_{e l}$, in Eq. 162 in order to control these unphysical modes. The goal of any process used to control hourglass modes is to alter the element stiffness matrix such that 
it is singular only with respect to rigid body motion, and non-singular with respect to constant-strain and hourglass modes. The technique used in controlling the hourglass modes arising from the element stiffness matrix for the diffusive mesh relaxer is to preserve the physical modes and couplings, seen in matrix $\mathbf{A}$, to maintain no interactions between the physical and unphysical modes in matrix $\mathbf{B}$, and to introduce terms to the unphysical modes so as to yield a nonsingular matrix $\mathbf{C}$ having a spectrum of eigenvalues of the same order of magnitude as that of the physical modes. The purpose of having the eigenvalues corresponding to the hourglass modes be of the same order of magnitude as those of the constant-strain modes is so that the condition number of the matrix not be greatly altered, and that the resulting matrix not simultaneously alter the response to legitimate modes.

In following the prescribed approach outlined above, matrix $\mathbf{A}$ and $\mathbf{B}$ will remain as described in Eq. 163 , and matrix $\mathbf{C}$ becomes

$$
C=\left[\begin{array}{cccc}
c_{5} & c_{56} & c_{57} & 0 \\
c_{56} & c_{6} & c_{67} & 0 \\
c_{57} & c_{67} & c_{7} & 0 \\
0 & 0 & 0 & c_{8}
\end{array}\right]
$$

where $c_{i j}$ needs to be determined. Based on the previous discussion, the $c_{i j}$ terms will need to be positive, and be of the order of the $a_{i j}$ terms, so that the resulting eigenvalues for the unphysical modes are of the same order as the physical modes. To determine the matrix elements for $\mathrm{C}$, it will be necessary to first write $\mathrm{M}_{e l}$, in terms of the original function $f$ instead of $u$, then expand this matrix, and analyze the effects of various values of $c_{i j}$. 
First, it is appropriate to restate the element variational integral, $I_{c l}$, from Eq. 161 , in terms of $f_{\alpha}$. Start with Eq. 161 , and substitute the vector notation for $\vec{u}$ specified by Eq. 155 to get

$$
I_{c l}=\frac{1}{8} w_{c l} \vec{u}^{T} M_{e l} \vec{u}=\frac{1}{64} w_{e l} \vec{f} \cdot T^{T} \cdot M_{e l} \cdot T \cdot \vec{f}=\frac{1}{64} w_{e l} \vec{f} \cdot M_{e l} \cdot \vec{f}
$$

where

$$
M_{e l}^{\prime}=T^{T} \cdot M_{e l} \cdot T
$$

or, using $\mathbf{M}_{e l}$, specified by Eq. 162 - Eq. 164 gives

$$
M_{e l}^{\prime}=T^{T}\left[\begin{array}{cccccccc}
0 & 0 & 0 & 0 & 0 & 0 & 0 & 0 \\
0 & a_{11} & a_{12} & a_{13} & 0 & 0 & 0 & 0 \\
0 & a_{12} & a_{22} & a_{23} & 0 & 0 & 0 & 0 \\
0 & a_{13} & a_{23} & a_{33} & 0 & 0 & 0 & 0 \\
0 & 0 & 0 & 0 & c_{5} & c_{56} & c_{57} & 0 \\
0 & 0 & 0 & 0 & c_{56} & c_{6} & c_{67} & 0 \\
0 & 0 & 0 & 0 & c_{57} & c_{67} & c_{7} & 0 \\
0 & 0 & 0 & 0 & 0 & 0 & 0 & c_{8}
\end{array}\right] T
$$

Next, expanding $M^{\prime}{ }_{e l}$ in terms of $\mathbf{T}$ specified by Eq. 155 results in 


$$
M_{e l}^{\prime}=\left[\begin{array}{llllllll}
m_{11} & m_{12} & m_{13} & m_{14} & m_{15} & m_{16} & m_{17} & m_{18} \\
m_{21} & m_{22} & m_{23} & m_{24} & m_{25} & m_{26} & m_{27} & m_{28} \\
m_{31} & m_{32} & m_{33} & m_{34} & m_{35} & m_{36} & m_{37} & m_{38} \\
m_{41} & m_{42} & m_{43} & m_{44} & m_{45} & m_{46} & m_{47} & m_{48} \\
m_{51} & m_{52} & m_{53} & m_{54} & m_{55} & m_{56} & m_{57} & m_{58} \\
m_{61} & m_{62} & m_{63} & m_{64} & m_{65} & m_{66} & m_{67} & m_{68} \\
m_{71} & m_{72} & m_{73} & m_{74} & m_{75} & m_{76} & m_{77} & m_{78} \\
m_{81} & m_{82} & m_{83} & m_{84} & m_{85} & m_{86} & m_{87} & m_{88}
\end{array}\right]
$$

where

$$
\begin{aligned}
& m_{11}=a_{11}+a_{22}+a_{33}+2\left(a_{12}+a_{13}+a_{23}\right)+c_{5}+c_{6}+c_{7}+2\left(c_{56}+c_{57}+c_{67}\right)+c_{8} \\
& m_{12}=m_{21}=-a_{11}+a_{22}+a_{33}+2 a_{23}-c_{5}+c_{6}-c_{7}-2 c_{57}-c_{8} \\
& m_{13}=m_{31}=-a_{11}-a_{22}+a_{33}-2 a_{12}+c_{5}-c_{6}-c_{7}-2 c_{67}+c_{8} \\
& m_{14}=m_{41}=a_{11}-a_{22}+a_{33}+2 a_{13}-c_{5}-c_{6}+c_{7}-2 c_{56}-c_{8} \\
& m_{15}=m_{51}=a_{11}+a_{22}-a_{33}+2 a_{12}+c_{5}-c_{6}-c_{7}-2 c_{67}-c_{8} \\
& m_{16}=m_{61}=-a_{11}+a_{22}-a_{33}-2 a_{13}-c_{5}-c_{6}+c_{7}-2 c_{56}+c_{8} \\
& m_{17}=m_{71}=-a_{11}-a_{22}-a_{33}-2\left(a_{12}+a_{13}+a_{23}\right)+c_{5}+c_{6}+c_{7} \\
& \quad+2\left(c_{56}+c_{57}+c_{67}\right)-c_{8} \\
& m_{18}=m_{81}=a_{11}-a_{22}-a_{33}-2 a_{23}-c_{5}+c_{6}-c_{7}-2 c_{57}+c_{8}
\end{aligned}
$$




$$
\begin{aligned}
& m_{22}=a_{11}+a_{22}+a_{33}+2\left(-a_{12}-a_{13}+a_{23}\right)+c_{5}+c_{6}+c_{7}+2\left(-c_{56}+c_{57}-c_{67}\right)+c_{8} \\
& m_{23}=m_{32}=a_{11}-a_{22}+a_{33}-2 a_{13}-c_{5}-c_{6}+c_{7}+2 c_{56}-c_{8} \\
& m_{24}=m_{42}=-a_{11}-a_{22}+a_{33}+2 a_{12}+c_{5}-c_{6}-c_{7}+2 c_{67}+c_{8} \\
& m_{25}=m_{52}=-a_{11}+a_{22}-a_{33}+2 a_{13}-c_{5}-c_{6}+c_{7}+2 c_{56}+c_{8} \\
& m_{26}=m_{62}=a_{11}+a_{22}-a_{33}-2 a_{12}+c_{5}-c_{6}-c_{7}+2 c_{67}-c_{8} \\
& m_{27}=m_{72}=a_{11}-a_{22}-a_{33}-2 a_{23}-c_{5}+c_{6}-c_{7}-2 c_{57}+c_{8} \\
& m_{28}=m_{82}=-a_{11}-a_{22}-a_{33}+2\left(a_{12}+a_{13}-a_{23}\right)+c_{5}+c_{6}+c_{7} \text {. } \\
& +2\left(-c_{56}+c_{57}-c_{67}\right)-c_{8} \\
& m_{33}=a_{11}+a_{22}+a_{33}+2\left(a_{12}-a_{13}-a_{23}\right)+c_{5}+c_{6}+c_{7}+2\left(-c_{56}-c_{57}+c_{67}\right)+c_{8} \\
& m_{34}=m_{43}=-a_{11}+a_{22}+a_{33}-2 a_{23}-c_{5}+c_{6}-c_{7}+2 c_{57}-c_{8} \\
& m_{35}=m_{53}=-a_{11}-a_{22}-a_{33}+2\left(-a_{12}+a_{13}+a_{23}\right)+c_{5}+c_{6}+c_{7} \\
& +2\left(-c_{56}-c_{57}+c_{67}\right)-c_{8} \\
& m_{36}=m_{63}=a_{11}-a_{22}-a_{33}+2 a_{23}-c_{5}+c_{6}-c_{7}+2 c_{57}+c_{8} \\
& m_{37}=m_{73}=a_{11}+a_{22}-a_{33}+2 a_{12}+c_{5}-c_{6}-c_{7}-2 c_{67}-c_{8} \\
& m_{38}=m_{83}=-a_{11}+a_{22}-a_{33}+2 a_{13}-c_{5}-c_{6}+c_{7}+2 c_{56}+c_{8} \\
& m_{44}=a_{11}+a_{22}+a_{33}+2\left(-a_{12}+a_{13}-a_{23}\right)+c_{5}+c_{6}+c_{7}+2\left(c_{56}-c_{57}-c_{67}\right)+c_{8} \\
& m_{45}=m_{54}=a_{11}-a_{22}-a_{33}+2 a_{23}-c_{5}+c_{6}-c_{7}+2 c_{57}+c_{8} \\
& m_{46}=m_{64}=-a_{11}-a_{22}-a_{33}+2\left(a_{12}-a_{13}+a_{23}\right)+c_{5}+c_{6}+c_{7} \\
& +2\left(c_{56}-c_{57}-c_{67}\right)-c_{8} \\
& m_{47}=m_{74}=-a_{11}+a_{22}+-a_{33}-2 a_{13}-c_{5}-c_{6}+c_{7}-2 c_{56}+c_{8} \\
& m_{48}=m_{84}=a_{11}+a_{22}-a_{33}-2 a_{12}+c_{5}-c_{6}-c_{7}+2 c_{67}-c_{8}
\end{aligned}
$$




$$
\begin{aligned}
& m_{55}=a_{11}+a_{22}+a_{33}+2\left(a_{12}-a_{13}-a_{23}\right)+c_{5}+c_{6}+c_{7}+2\left(-c_{56}-c_{57}+c_{67}\right)+c_{8} \\
& m_{56}=m_{65}=-a_{11}+a_{22}+a_{33}-2 a_{23}-c_{5}+c_{6}-c_{7}+2 c_{57}-c_{8} \\
& \dot{m}_{57}=m_{75}=-a_{11}-a_{22}+a_{33}-2 a_{12}+c_{5}-c_{6}-c_{7}-2 c_{67}+c_{8} \\
& m_{58}=m_{85}=a_{11}-a_{22}+a_{33}-2 a_{13}-c_{5}-c_{6}+c_{7}+2 c_{56}-c_{8} \\
& m_{66}=a_{11}+a_{22}+a_{33}+2\left(-a_{12}+a_{13}-a_{23}\right)+c_{5}+c_{6}+c_{7}+2\left(c_{56}-c_{57}-c_{67}\right)+c_{8} \\
& m_{67}=m_{76}=a_{11}-a_{22}+a_{33}+2 a_{13}-c_{5}-c_{6}+c_{7}-2 c_{56}-c_{8} \\
& m_{68}=m_{86}=-a_{11}-a_{22}+a_{33}+2 a_{12}+c_{5}-c_{6}-c_{7}+2 c_{67}+c_{8} \\
& m_{77}=a_{11}+a_{22}+a_{33}+2\left(a_{12}+a_{13}+a_{23}\right)+c_{5}+c_{6}+c_{7}+2\left(c_{56}+c_{57}+c_{67}\right)+c_{8} \\
& m_{78}=m_{87}=-a_{11}+a_{22}+a_{33}+2 a_{23}-c_{5}+c_{6}-c_{7}-2 c_{57}-c_{8} \\
& m_{88}=a_{11}+a_{22}+a_{33}+2\left(-a_{12}-a_{13}+a_{23}\right)+c_{5}+c_{6}+c_{7}+2\left(-c_{56}+c_{57}-c_{67}\right)+c_{8}
\end{aligned}
$$

Looking at the equations in Eq. 169 for $m_{i j}$ and the unit cube with the node numbers in Eq. 18, a few symmetric patterns become evident. The three dimensional diagonal nodes, nodes 1 and 7 , nodes 2 and 8 , nodes 3 and 5 , and nodes 4 and 6 , are symmetric, except for the node couplings between these nodes. In matrix form, this symmetry allows $M^{\prime}{ }_{e l}$ to be simplified to

$$
M^{\prime}{ }_{e l}=\left[\begin{array}{llllllll}
m_{11} & m_{12} & m_{13} & m_{14} & m_{15} & m_{16} & m_{17} & m_{18} \\
m_{12} & m_{22} & m_{23} & m_{24} & m_{25} & m_{26} & m_{18} & m_{28} \\
m_{13} & m_{23} & m_{33} & m_{34} & m_{35} & m_{36} & m_{15} & m_{25} \\
m_{14} & m_{24} & m_{34} & m_{44} & m_{36} & m_{46} & m_{16} & m_{26} \\
m_{15} & m_{25} & m_{35} & m_{36} & m_{33} & m_{34} & m_{13} & m_{23} \\
m_{16} & m_{26} & m_{36} & m_{46} & m_{34} & m_{44} & m_{14} & m_{24} \\
m_{17} & m_{18} & m_{15} & m_{16} & m_{13} & m_{14} & m_{11} & m_{12} \\
m_{18} & m_{28} & m_{25} & m_{26} & m_{23} & m_{24} & m_{12} & m_{22}
\end{array}\right]
$$


It is instructive to look again at the independent displacement modes in Fig. 17, and more specifically the hourglass modes. Notice that the magnitude of the three dimensional diagonals for these modes seem to affect the amount of distortion of the hourglassing element. Large values for the three dimensional diagonal couplings then, would appear to dominate the displacements. Eliminating these diagonal couplings in two dimensions has proven successful in controlling unphysical modes ${ }^{21,37}$. Following the same idea presented in these papers, it is appropriate to set

$$
m_{17}=m_{28}=m_{35}=m_{46}=0,
$$

which should be a reasonable approach to controlling the hourglass modes in the diffusive mesh relaxer.

Using Eq. 171 , the $c_{i j}$ terms in Eq. 164 can be determined. The equations to solve are

$$
\begin{aligned}
& m_{17}=0 \\
& m_{28}=0 \\
& m_{35}=0 \\
& m_{46}=0
\end{aligned}
$$

From Eq. 164 , it is reasonable to set the diagonal terms of $\mathbf{C}$ to appropriate values of $a_{i i}$, and the off diagonals of $\mathrm{C}$ to $a_{i j}, i \neq j$. Eq. 172 , then, reduces to two sets of equations. First,

$$
-a_{11}-a_{22}-a_{33}+c_{5}+c_{6}+c_{7}-c_{8}=0 .
$$

One obvious solution is to let 


$$
\begin{aligned}
& c_{5}=a_{11} \\
& c_{6}=a_{22} \\
& c_{7}=a_{33} \\
& c_{8}=0
\end{aligned}
$$

This solution, however, violates several of the initial conditions set on $\mathbf{C}$, specifically that the terms in $\mathbf{C}$ be positive, and that the resulting eigenvalues not be zero. The next solution is to allow

$$
\begin{aligned}
& c_{5}=a_{11}+a_{22} \\
& c_{6}=a_{22}+a_{33} \\
& c_{7}=a_{11}+a_{33} \\
& c_{8}=a_{11}+a_{22}+a_{33} .
\end{aligned}
$$

Eq. 175 satisfies Eq. 173 and meets the requirements for setting the values for $\mathrm{C}$. The second set of equations to solve are

$$
\begin{aligned}
-2\left(a_{12}+a_{13}+a_{23}\right)+2\left(c_{56}+c_{57}+c_{67}\right) & =0 \\
2\left(-a_{12}+a_{13}+a_{23}\right)+2\left(-c_{56}-c_{57}+c_{67}\right) & =0 \\
2\left(a_{12}+a_{13}-a_{23}\right)+2\left(-c_{56}+c_{57}-c_{67}\right) & =0 \\
2\left(a_{12}-a_{13}+a_{23}\right)+2\left(c_{56}-c_{57}-c_{67}\right) & =0
\end{aligned}
$$

which are satisfied when

$$
\begin{aligned}
& c_{67}=a_{12} \\
& c_{57}=a_{23} . \\
& c_{56}=a_{13} .
\end{aligned}
$$

Substituting the above values for $c_{i j}$ into Eq. 167 and the resulting equations for $m_{i j}$ spec- 
ified by Eq. 169 gives

$$
\begin{aligned}
& m_{11}=m_{77}=4\left(a_{11}+a_{22}+a_{33}+a_{12}+a_{13}+a_{23}\right) \\
& m_{12}=m_{21}=m_{78}=m_{87}=-4 a_{11} \\
& m_{13}=m_{31}=m_{57}=m_{75}=-4 a_{12} \\
& m_{14}=m_{41}=m_{67}=m_{76}=-4 a_{22} \\
& m_{15}=m_{51}=m_{37}=m_{73}=-4 a_{33} \\
& m_{16}=m_{61}=m_{47}=m_{74}=-4 a_{13} \\
& m_{17}=m_{71}=0 \\
& m_{18}=m_{81}=m_{27}=m_{72}=-4 a_{23} \\
& m_{22}=m_{88}=4\left(a_{11}+a_{22}+a_{33}+-a_{12}-a_{13}+a_{23}\right) \\
& m_{23}=m_{32}=m_{58}=m_{85}=-4 a_{22} \\
& m_{24}=m_{42}=m_{68}=m_{86}=4 a_{12} \\
& m_{25}=m_{52}=m_{38}=m_{83}=4 a_{13} \\
& m_{26}=m_{62}=m_{48}=m_{84}=-4 a_{33} \\
& m_{28}=m_{82}=0 \\
& m_{33}=m_{55}=4\left(a_{11}+a_{22}+a_{33}+a_{12}-a_{13}-a_{23}\right) \\
& m_{34}=m_{43}=m_{56}=m_{65}=-4 a_{11} \\
& m_{35}=m_{53}=0 \\
& m_{36}=m_{63}=m_{45}=m_{54}=4 a_{23} \\
& m_{44}=4\left(a_{11}+a_{22}+a_{33}+-a_{12}+a_{13}-a_{23}\right) \\
& m_{46}=m_{64}=0
\end{aligned}
$$

The numerical implementation of the diffusive mesh relaxer described in Chapter 3 
has now been modified to control hourglass modes in the resulting grid. The initial algorithm consisted of solving for the new positions of node $\alpha$ using Eq. 179 below.

$$
\begin{aligned}
f_{\alpha} & =\frac{1}{M_{\alpha \alpha}}\left(\sum_{\beta \neq \alpha} M_{\alpha \beta} f_{\beta}\right) \\
& =\frac{\sum_{\text {all contributing elements }}\left(w_{\xi 00}\left[a_{11}\right]+w_{0 \eta 0}\left[a_{22}\right]+w_{00 \zeta}\left[a_{33}\right]\right) f_{\beta}}{\sum_{\text {all contributing elements }}\left(w_{\xi 00}\left[a_{1 j}\right]+w_{0 \eta 0}\left[a_{2 j}\right]+w_{00 \zeta}\left[a_{3 j}\right]\right)}
\end{aligned}
$$

The modified algorithm applies the same general format in that

$$
f_{\alpha}=\frac{1}{M_{\alpha \alpha}}\left(\sum_{\beta \neq \alpha} M_{\alpha \beta} f_{\beta}\right)=\left\{\left(\frac{M_{\alpha \beta} f_{\beta}}{M_{\alpha \alpha}}\right)_{e l 1}+\left(\frac{M_{\alpha \beta} f_{\beta}}{M_{\alpha \alpha}}\right)_{e l 2}+\ldots\right\}
$$

With the hourglass modification, however, $\left(\frac{M_{\alpha \beta} f_{\beta}}{M_{\alpha \alpha}}\right)_{e l i}$ is different depending on which element node $\alpha$ is. For example, if node $\alpha$ is node 1 with regard to the contributing element's connectivity, as seen in Fig. 18 , then using the matrix $M_{\alpha \beta}$ defined in Eq. 178

$$
\left(\frac{M_{\alpha \beta} f_{\beta}}{M_{\alpha \alpha}}\right)_{e l i}=\frac{w_{\alpha \beta}\left(a_{11} \dot{f}_{12}+a_{22} f_{14}+a_{33} f_{15}+a_{12} f_{13}+a_{23} f_{18}+a_{13} f_{16}\right)}{w_{\alpha \beta}\left(a_{11}+a_{22}+a_{33}+a_{12}+a_{23}+a_{13}\right)}
$$

If node $\alpha$ is node 2 , then

$$
\left(\frac{M_{\alpha \beta} f_{\beta}}{M_{\alpha \alpha}}\right)_{e l i}=\frac{w_{\alpha \beta}\left(a_{11} f_{21}+a_{22} f_{23}+a_{33} f_{26}-a_{12} f_{24}+a_{23} f_{27}-a_{13} f_{25}\right)}{w_{\alpha \beta}\left(a_{11}+a_{22}+a_{33}-a_{12}+a_{23}-a_{13}\right)}
$$

The remaining equations for the contributing elements, $\left(\frac{M_{\alpha \beta} f_{\beta}}{M_{\alpha \alpha}}\right)_{e l i}$, are determined in a similar manner. 


\section{Chapter 5: Lagrangian Mechanics}

\section{Introduction}

Before discussing the application and results of the diffusive mesh relaxer, it is beneficial to review Lagrangian mechanics. Reiterating from Chapter 1, the diffusive mesh relaxer is part of a Continuum mechanics application, which is using the ALE (Arbitrary Lagrangian-Eulerian) computational technique. An ALE formulation consists of two steps, first the complete Lagrangian calculation and then a modified Eulerian, or advection step.

The following sections describe the basic laws of Continuum mechanics and what is meant by the Lagrangian formulation.

\section{Continuum Mechanics}

Investigating material behavior sometimes demands studying the material as a whole. The material is assumed to be continuously distributed throughout its volume, and remains continuous under the action of external affects, with no cracks or holes forming in the interior. Materials satisfying this condition are said to be a continuum ${ }^{13}$, and the study of the motion and deformation of such materials, called bodies, is continuum mechanics. External affects that influence these continuous bodies appear as forces, velocities, and displacements which arise from contact with other bodies, gravitational forces, thermal changes, chemical interactions, and other environmental changes. In essence, the theory of continuum mechanics is built upon two strong foundations. The first is the basic laws of motion and the second is a constitutive theory governing the material characteristics. 
Fig. 19 shows a continuous material and its associated volume, surface and normal vectors. In this figure, $d V$ represents the volume differential, where $\vec{\nu}$ is the velocity of that volume, and $\vec{b}$ is the body force acting on the volume. The surface differential is $d S$, to which $\vec{n}$ is normal to this surface and $\vec{\sigma}$ is the stress vector acting on the surface element.

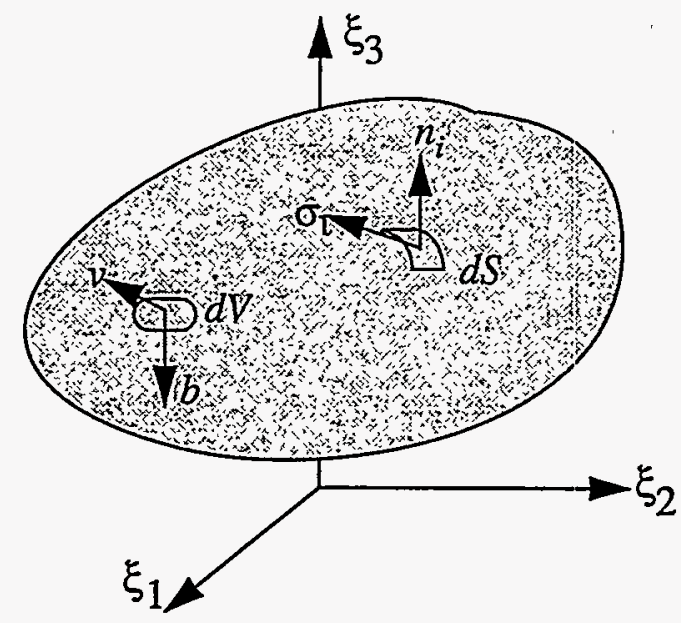

Fig. 19: Continuous Material

The amount of mass in a portion of the continuum occupying the spatial volume $V$ at time $t$ is given by 22

$$
m=\int_{V} \rho(\vec{x}, t) d V
$$

Newton's law of motion ${ }^{13}$, specified by Euler for a continuum, states that the material rate of change of linear momentum $\dot{\vec{P}}$ is equal to the total applied force $\vec{F}$ acting on the body,

$$
\dot{\vec{P}}=\frac{d}{d t} \grave{P}=\vec{F} .
$$


Linear momentum of the body is given by

$$
\grave{P}=\int_{V} \rho \grave{v} d V
$$

where $\rho=\rho(\vec{x}, t)$ is the density of the material which is a function of position and time, $\bar{\nu}$ is the velocity vector and the integration is over the volume $V$.

The total external forces are given by the sum of the body forces acting on the volume of the body, and the surface forces or stresses acting on the surface of the body,

$$
\vec{F}=\vec{F}_{b}+\vec{F}_{s}
$$

The body forces, like gravitational forces, acting over the volume of the material are given by

$$
\vec{F}_{b}=\int_{V} \vec{b} d V
$$

where $\vec{b}$ is the body force vector per unit volume. The surface forces created by stresses acting on the surface elements are given by

$$
\vec{F}_{s}=\int_{S} \vec{\partial} d S=\int_{S} \sigma_{i j} n_{j} d S
$$

where $\partial$ is the stress vector acting on surface element $d S$. whose outward normal is $\vec{n}$, and $\sigma_{i j}$ is the stress tensor where $\sigma_{i}=\sigma_{i j} n_{j}$. Combining Eq. 185, Eq. 187 and Eq. 188 , and applying these equations to Eq. 184 gives the following equation for Newton's law of motion: 


$$
\frac{d}{d t} \int_{V} \rho v_{i} d V=\int_{V} b_{i} d V+\int_{S} \sigma_{i j} n j d S
$$

Notice that the left integral in Eq. 189 is the material derivative of linear momentum, which is the time rate of change of linear momentum in a continuum with respect to the specific particles of the moving continuum.

\section{Lagrangian Frame of Reference}

In the Eulerian frame of reference, the coordinates are spatial, and concern themselves with a particular fixed region of space occupied by the continuum.

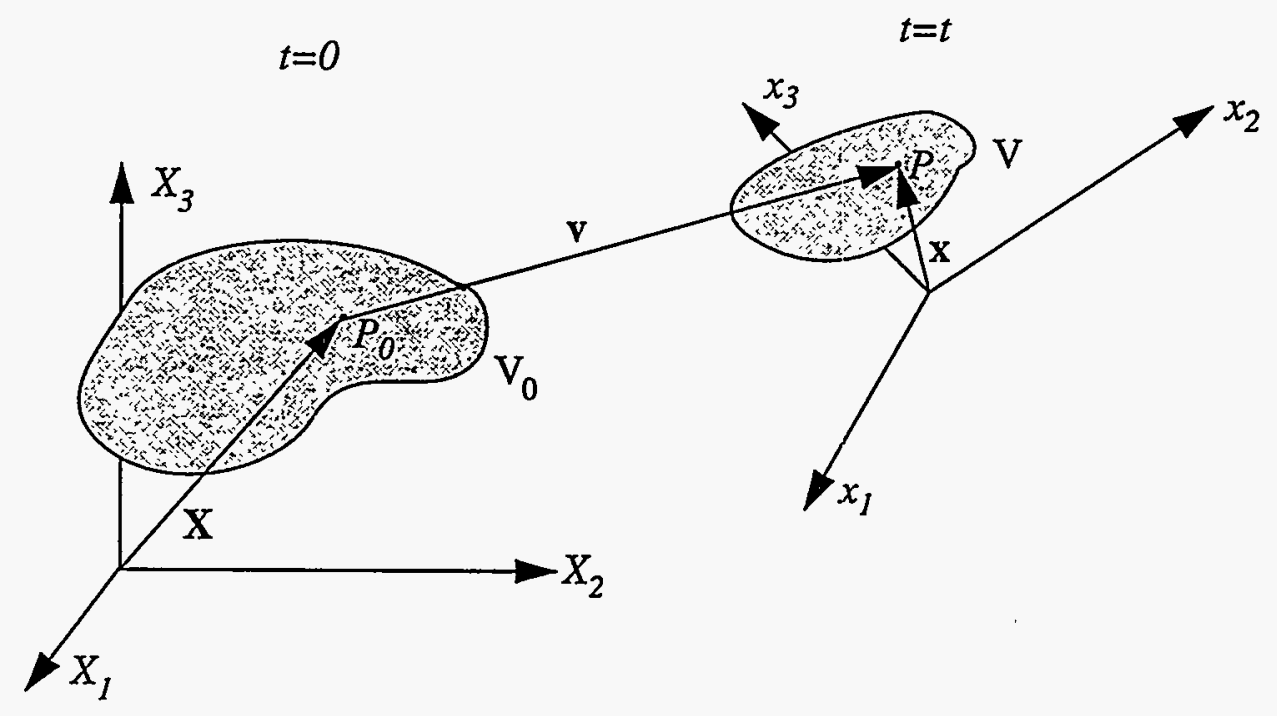

Fig. 20: Eulerain and Lagrangian Frames of Reference

In this case, the motion of the continuum is given through equations of the form

$$
X_{i}=X_{i}\left(x_{1}, x_{2}, x_{3}, t\right)
$$

where $X_{i}$ and $x_{i}$ are illustrated in Fig. 20 .

Within the Lagrangian frame of reference, the coordinates are material based, concerning themselves with specific fixed particles of the continuum. In this case, the motion of the continuum is represented by 


$$
x_{i}=x_{i}\left(X_{1}, X_{2}, X_{3}, t\right)
$$

and the velocity of the material is given by

$$
v_{i}=v_{i}\left(x_{i}, t\right)=\frac{\partial x_{i}}{\partial t}
$$

If Eq. 190 and Eq. 191 are one-to-one mappings with continuous partial derivatives, then these mappings are the unique inverse of each other. The concept of one-to-one mappings has been discussed in earlier chapters. Recall that a necessary and sufficient condition for the inverse functions to exist is that $j$, the determinant of the Jacobian Matrix, J, does not vanish.

Given that $j$ is non-zero, the Lagrangian continuity equation and then the Lagrangian equation of motion for a continuum can be derived. Applying the conservation of mass principle and the definition in Eq. 183 gives

$$
\int_{V_{0}} \rho_{0}(\grave{X}, 0) d V_{0}=\int_{V} \rho(\grave{x}, t) d V
$$

where $V$ is the volume occupied by the material and $\rho$ the mass density at time $t$, which occupied $V_{0}$ with density $\rho_{0}$ at time $t=0$. When the continuum moves from some initial configuration at time $t=0$ to a later time $t$, it will occupy a differential volume element, $d V_{0}$ in the initial state, and $d V$ at time $t$. Due to this motion, the volume element becomes distorted, but because the motion is assumed continuous, and the material is a continuum, the volume element does not break up. Then

$$
d V=d X_{1} d X_{2} d X_{3}=\varepsilon_{i j k} \frac{\partial x_{i}}{\partial X_{1}} d X_{1} \frac{\partial x_{j}}{\partial X_{2}} d X_{2} \frac{\partial x_{k}}{\partial X_{3}} d X_{3}=j d V_{0}
$$


Using the definition of Lagrangian coordinates from Eq. 191 and Eq. 194 , the conservation of mass integral given in Eq. 193 can be rewritten as

$$
\int_{V_{0}} \rho_{0}(\vec{X}, 0) d V_{0}=\int_{V} \rho(\vec{x}(\vec{X}, t), t) j d V_{0}=\int_{V_{0}} \rho_{0}(\vec{X}, t) j d V_{0}
$$

The above Eq. 195 must hold for arbitrary volumes, so the integrals vanish to yield

$$
\rho_{0}=j \rho \text { or } \frac{d}{d t}[j \rho]=0,
$$

which is the Lagrangian differential form of the continuity equation.

The Lagrangian equation of motion is derived by taking the material derivative of the left side of Eq. 189 , and using the material coordinate specified in Eq. 191 . The resulting equation is

$$
\frac{d}{d t} \int_{V} \rho v_{i} d V=\int_{V} \frac{d}{d t}\left[\rho v_{i} d V\right]=\int_{V}\left[\frac{\partial}{\partial t} \rho v_{i}+\frac{\partial}{\partial X_{j}} \rho v_{i} \frac{d X}{d t}\right] \frac{d}{d t}(d V)
$$

Since $\vec{X}$ is independent of time,

$$
\frac{d X}{d t}=0
$$

Because the volume and thus the differential volume element of the continuum in the Lagrangian frame of reference is changing with time, the rate of change for these variables is non-zero and can be written as

$$
\frac{d}{d t}(d V)=\frac{d}{d t}\left(j d V_{0}\right)=\frac{d j}{d t} d V_{0}+j \frac{d V_{0}}{d t}=\frac{d j}{d t} d V_{0}=j \frac{\partial v_{i}}{\partial x_{i}} d V_{0}=\frac{\partial v_{i}}{\partial x_{i}} d V
$$


since $d V_{0}$ is time independent and the material derivative of the Jacobian is

$$
\frac{d j}{d t}=j \frac{\partial v_{i}}{\partial x_{i}}
$$

Applying Eq. 194 , Eq. 196 and Eq. 199 to the left side of Eq. 197 gives

$$
\frac{d}{d t} \int_{V} \rho v_{i} d V=\frac{d}{d t} \int_{V_{0}} \rho v_{i} j d V_{0}=\int_{V_{0}}\left[v_{i} \frac{d}{d t}(\rho j)+\rho j \frac{d v_{i}}{d t}\right] d V_{0}=\int_{V}^{d v_{i}} \rho d V
$$

and substituting Eq. 198 - Eq. 201 back into the equation of motion given by Eq. 197 yields

$$
\int_{V}^{d v_{i}} \frac{d V}{d t} \rho \int_{V}\left(\sigma_{i j, j}+b_{i}\right) d V
$$

which is the Lagrangian equation of motion. Compare this result with that specified for the Eulerian equation of motion given in Eq. 189 .

The purpose of this chapter was to introduce the concept of Lagrangian mechanics by deriving the Lagrangian differential form of the continuity equation and the Lagrangian equation of motion. 


\section{Chapter 6: Application and Results}

Now that the foundation for the diffusive mesh relaxer has been laid, the application of this algorithm can be discussed. First, the diffusive mesh relaxer is applied to a two dimensional, structured problem to validate the idea of using a diffusive, geometric based algorithm. Then, the relaxer is integrated into an ALE finite element numerical simulation package, ALE3D, and applied to several three dimensional applications. A brief overview of ALE3D is first given, followed by the applications and their results.

\section{Preliminary 2D Structured Mesh Findings}

To determine the feasibility of using a geometric weight for controlling the movement of grid lines, the elliptic equations

$$
\begin{aligned}
& \nabla \lambda_{1} \nabla \xi(x, y)=0 \\
& \nabla \lambda_{2} \nabla \eta(x, y)=0
\end{aligned}
$$

were solved on a 2D structured mesh as shown in Fig. 21. $\xi$ and $\eta$ are the logical coordinate lines discussed in previous chapters, and $\lambda_{1}$ and $\lambda_{2}$ are the geometric weights 
described in Eq. 205.

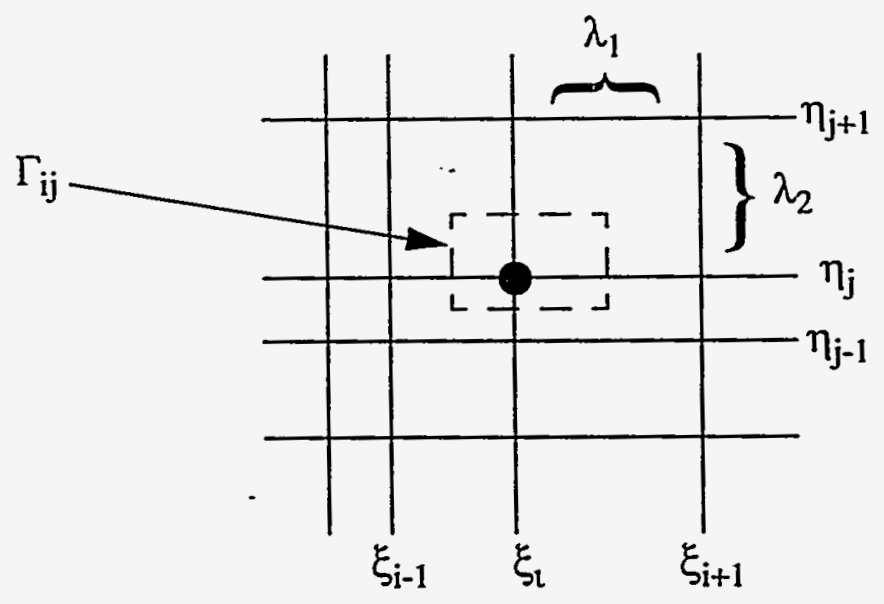

Fig. 21: Two Dimensional Structured Mesh

The equations were discretized using the finite volume method which applies one of Green's Identities, resulting in the boundary integral

$$
\begin{aligned}
& \int_{\Gamma_{i j}}\left[\lambda_{1_{i j}} \frac{\partial \xi}{\partial x} \cos (\mathbf{n}, x)+\lambda_{1_{i j}} \frac{\partial \xi}{\partial y} \cos (\mathbf{n}, y)\right] d \Gamma_{i j}=0 \\
& \int_{\Gamma_{i j}}\left[\lambda_{2 i j} \frac{\partial \eta}{\partial x} \cos (\mathbf{n}, x)+\lambda_{2_{i j}} \frac{\partial \eta}{\partial y} \cos (\mathbf{n}, y)\right] d \Gamma_{i j}=0
\end{aligned}
$$

where $\Gamma$ is the boundary of the finite volume, and $\cos (\mathbf{n}, x / y)$ is the cosine of the normal to the $x / y$ axis, perpendicular to the boundary $\Gamma$. The geometric weights are defined as

$$
\lambda_{1_{i j}}=\frac{1}{\left|x_{i}-x_{i-1}\right|} \text { and } \lambda_{2_{i j}}=\frac{1}{\left|y_{j}-y_{j-1}\right|} \text {. }
$$

These integrals in Eq. 204 are approximated by the midpoint rule and the resulting symmetric, positive definite matrices are solved with the preconditioned conjugate gradient method.

The original mesh, seen in Fig. 22 , is relaxed using two different mesh relaxers, the diffusive mesh relaxer and the Winslow-Crowley or Equipotential method ${ }^{9}, 38,39$, and 
are seen in Fig. 23.

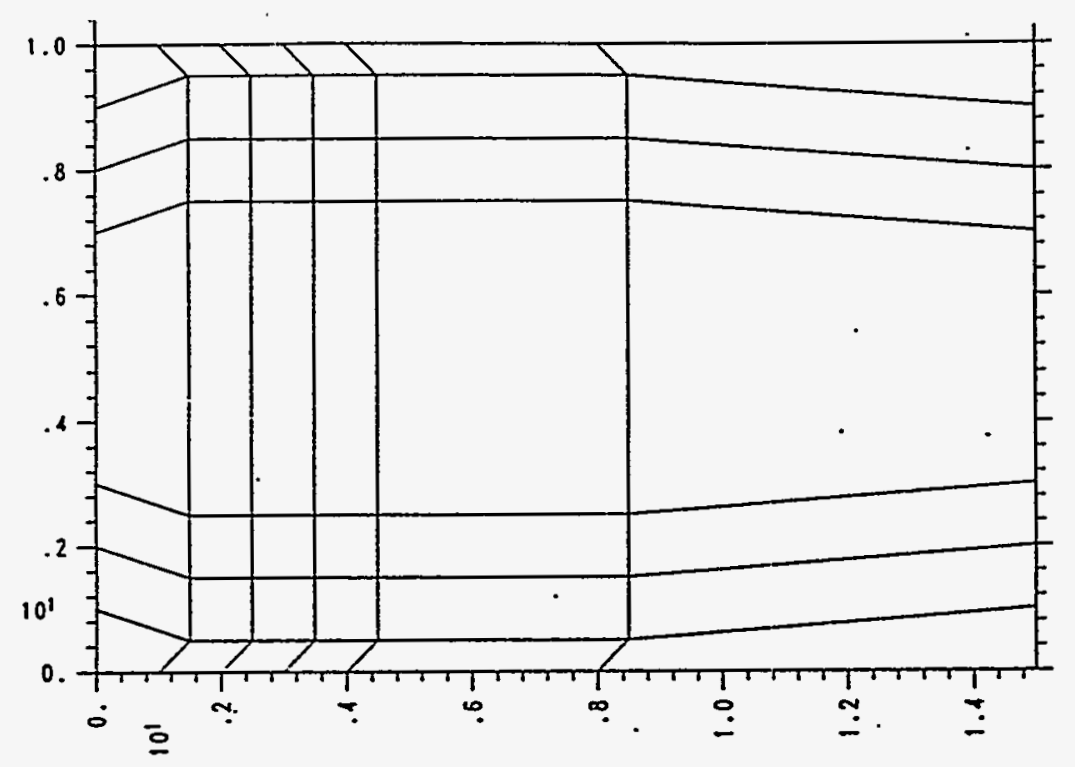

Fig. 22: Original Two Dimensional Structured Physical Mesh 


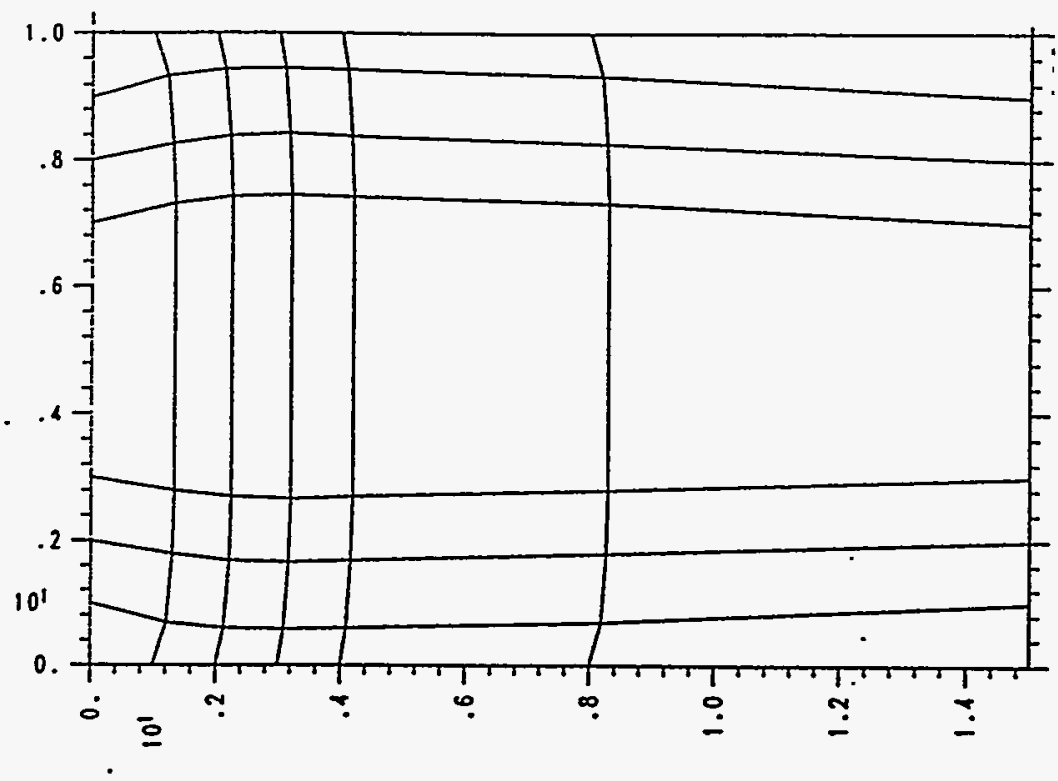

Diffusive Geometric Mesh Relaxer

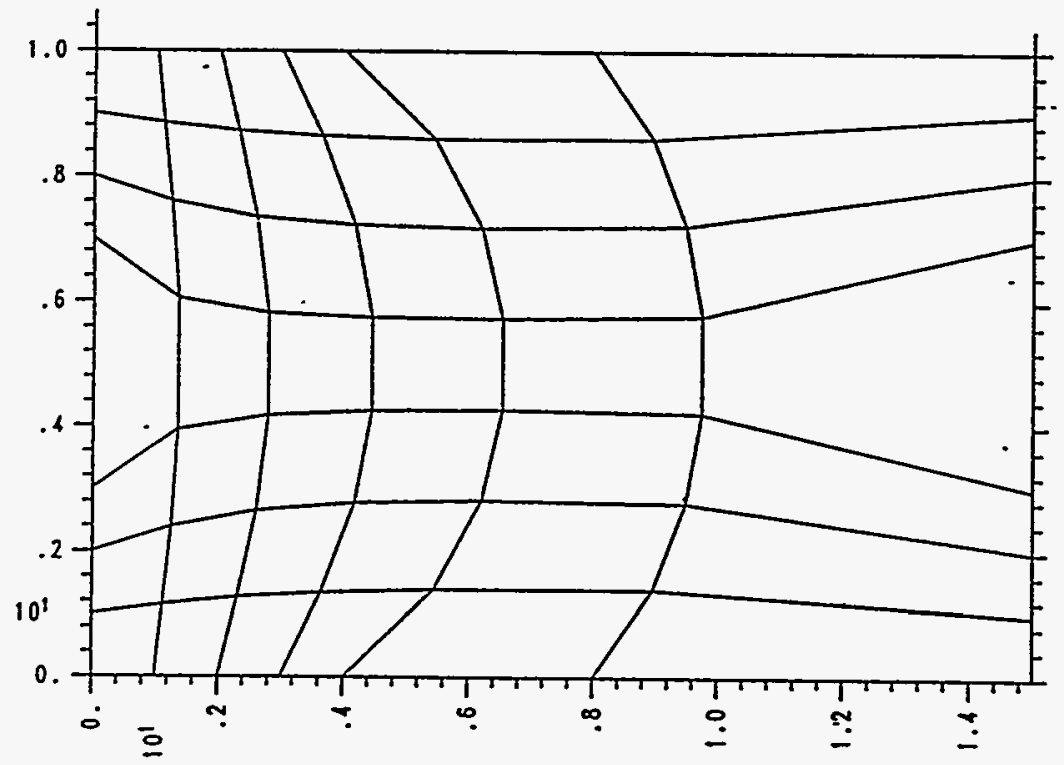

Equipotential Mesh Relaxer

Fig. 23: Relaxed Two Dimensional Structured Physical Mesh 
The Equipotential relaxer smooths the zoning, destroying the original characteristics of the mesh, whereas the diffusive geometric relaxer retained these characteristics. These initial findings demonstrated that the diffusive geometric mesh relaxer appeared able to maintain the original mesh zoning structure, giving the necessary encouragement to proceed into the three dimensional unstructured arena.

\section{ALE3D \\ The three dimensional, unstructured diffusive mesh relaxer is installed in ALE3D ${ }^{26,8}$ ,16 , an arbitrary Lagrange/Eulerian (ALE) three-dimensional Continuum Mechanics code system. ALE3D is a finite element code that treats fluid and elastic-plastic response on an unstructured grid. Examples of problems simulated by ALE3D include impact problems and metal deformation such as casting, forging and rolling.This grid consists of arbitrarily connected hexahedral, shell and beam elements, and can be constructed from disjoint blocks of elements which interact at the boundaries via slide surfaces or other types of boundary conditions.}

The interaction at slide surfaces can consist of pure sliding in which there are no tangential forces on the interface nodes, tied sliding in which the nodes are inhibited from sliding, or friction sliding in which a coulomb friction algorithm is applied to the interacting nodes. If a void, or open gap between the two surfaces, is not present, the forces on either side of the slide surface are accumulated and used to produce a net acceleration of the nodes on the surface consistent with the center-of-mass motion.

An ALE formulation consists of two steps, first the complete Lagrangian calculation and then a modified Eulerian, or advection step. In the Lagrangian phase, nodal forces 
are accumulated and the updated nodal acceleration is computed. Next, the zonal quantities are calculated, and then the velocities and nodal positions are updated.

At the end of the Lagrangian calculation, the user may chose to run ALE3D in a pureLagrangian mode, and thus continues with the next time step. If an advection mode is chosen, then two more steps in the computation are taken. The mesh is regenerated, or relaxed, to alleviate any tangled or irregular zones created by the Lagrangian calculation.This new mesh needs to be smooth, with no bow-ties or distorted elements, and no drastic changes in zone size. The existing mesh relaxation technique aside from the diffusive, geometric mesh relaxer involves a finite element based Equipotential method ${ }^{37}$. ALE3D has the ability to handle multimaterial elements, thus allowing relaxation to take place across material boundaries. Next, the zonal quantities are remapped from the old to the new relaxed mesh using various conservative convection schemes. ${ }^{41}$

\section{Test Problem: A Cube}

The diffusive mesh relaxer is first applied to a simple $2 \mathrm{~cm}$ by $2 \mathrm{~cm}$ cube, using various zoning structures. The first test of the algorithm uses the simple zoning structure seen in 
Fig. 24 .

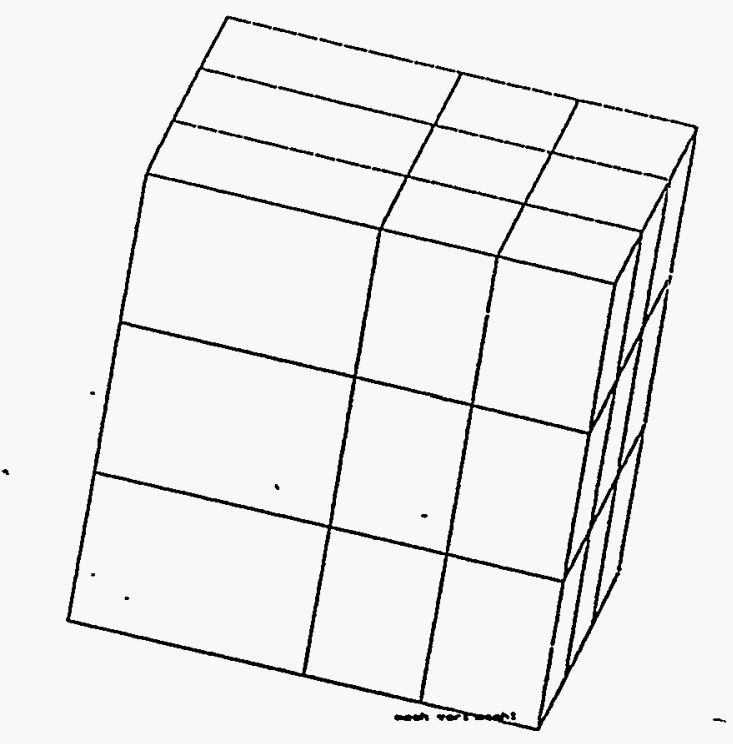

Fig. 24: Simple Cube Test Case

To allow for better comparison of the forthcoming results, an orthogonal slice is taken through the cube, and the $2 \mathrm{D}$ plot of this slice is shown in Fig. 25 .

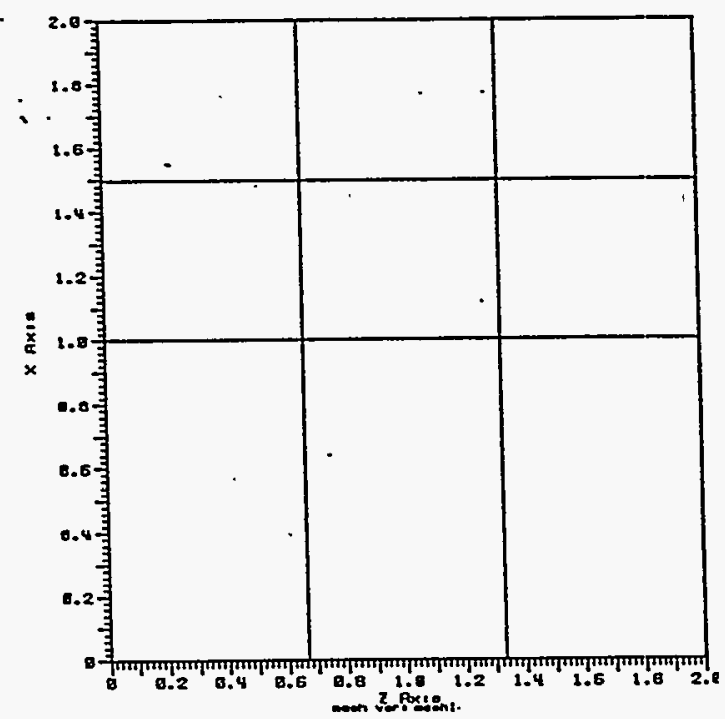

Fig. 25: Orthogonal Slice of Simple Cube Test Case 
Remember that the weights, discussed in Chapter 3, have the general form of

$$
\begin{aligned}
& W_{x_{\alpha}}=\left(\frac{1}{|x(\alpha)-x(\beta)|}\right)^{r} \\
& W_{y_{\alpha}}=\left(\frac{1}{|y(\alpha)-y(\beta)|}\right)^{r} \\
& W_{z_{\alpha}}=\left(\frac{1}{|z(\alpha)-z(\beta)|}\right)^{r} .
\end{aligned}
$$

where $r$ is an arbitrary number. The resulting mesh for several values of $r$ are shown in Fig. 26 through Fig. 30 .

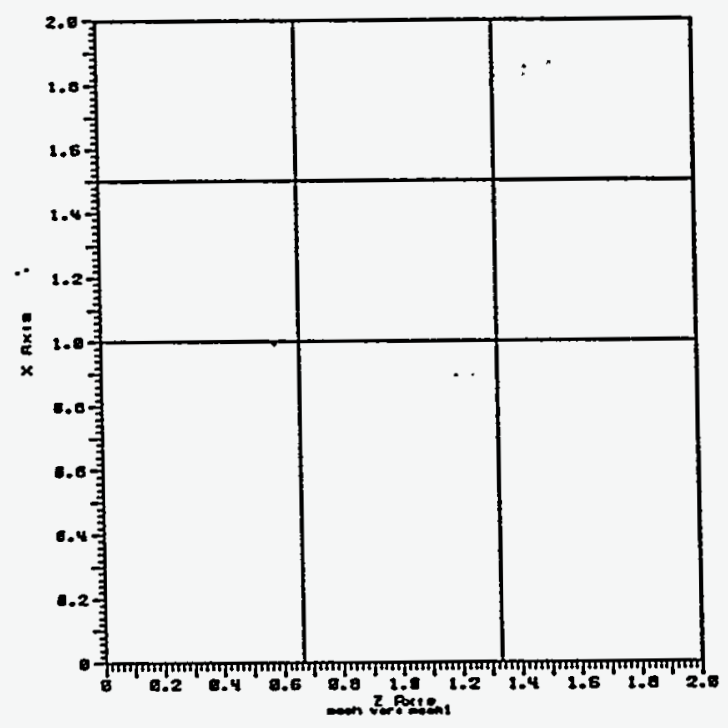

Fig. 26: Orthogonal Slice of Diffusive Mesh Relaxer with $r=1$. 


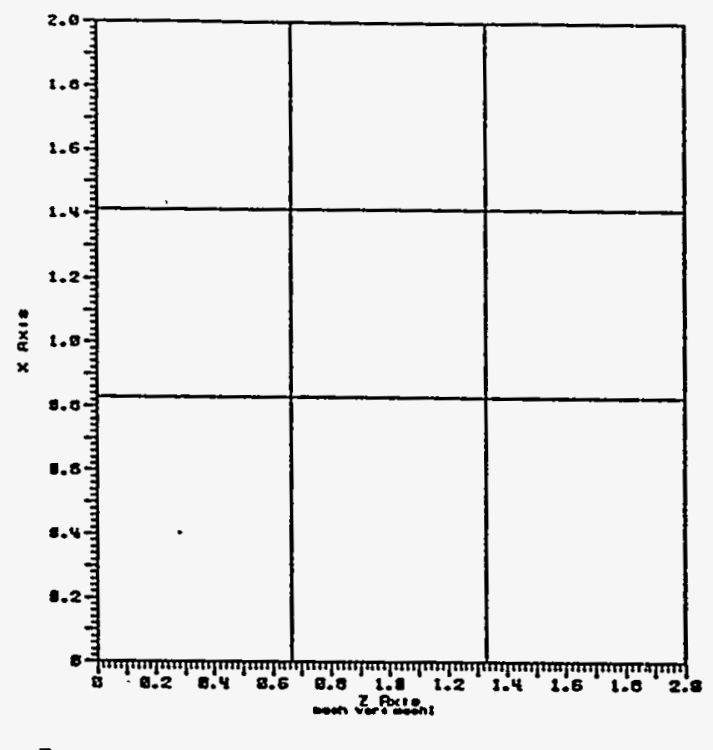

Fig. 27: Orthogonal Slice of Diffusive Mesh Relaxer with $r=1 / 2$.

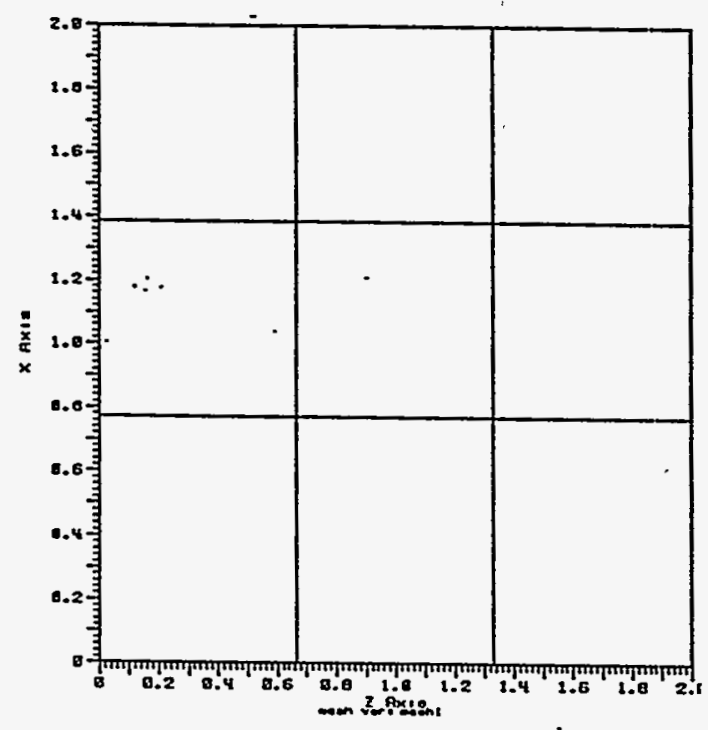

Fig. 28: Orthogonal Slice of Diffusive Mesh Relaxer with $r=1 / 3$. 


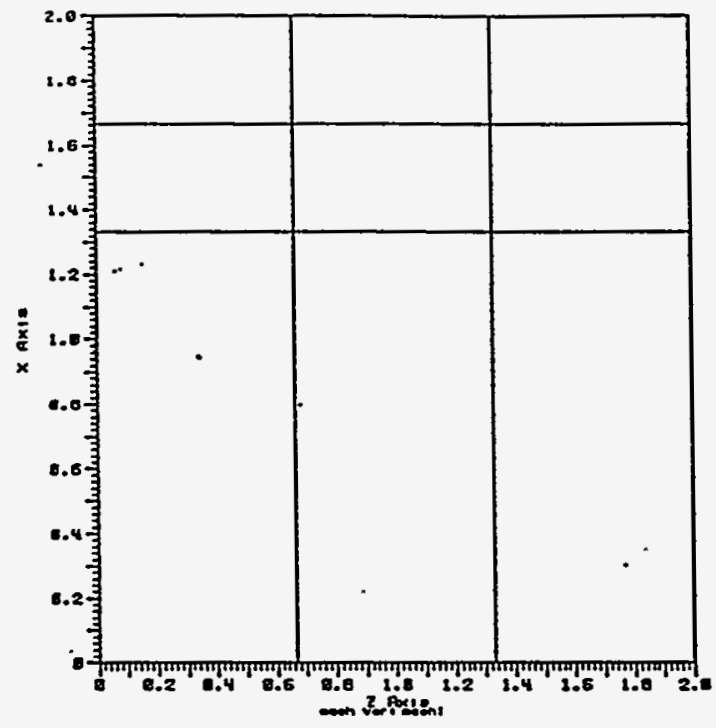

Fig. 29: Orthogonal Slice of Diffusive Mesh Relaxer with $r=2$.

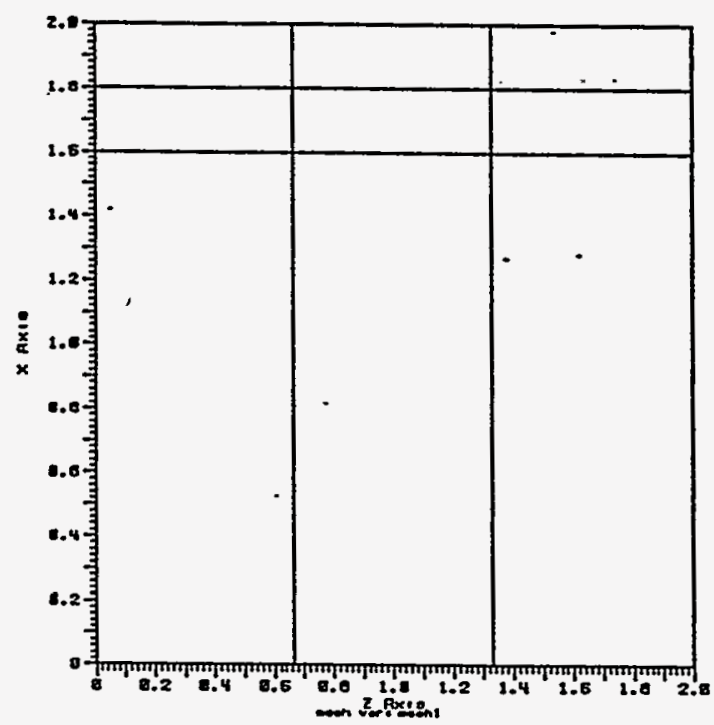

Fig. 30: Orthogonal Slice of Diffusive Mesh Relaxer with $r=3$.

For comparison, it is instructive to relax the original mesh seen in Fig. 24 using the Win- 
slow-Crowley or Equipotential method ${ }^{9,38,39}$. The resulting mesh is seen in Fig. 31 .

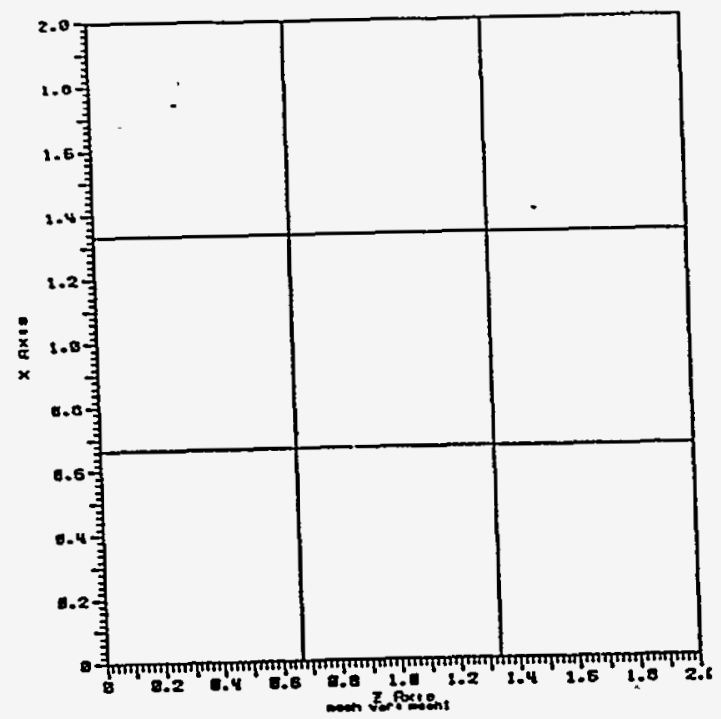

Fig. 31: Orthogonal Slice of Equipotential Mesh Relaxer.

Several observations can be made about these results. As the value for $r$ decreased, the resulting mesh structure approached that of the Equipotential mesh relaxer in that the zones attained equal volume. Studying the equations for the weighting terms given in Eq. 206 - Eq. 208 shows that for the simple cube example, these weights approach 1 as $r$ decreases, and thus the resulting elliptic equation being solved by the diffusive mesh relaxer is Laplace's equation, the basis for the Equipotential algorithm. The opposite effect occurs when $r$ increases. Again, examining the equations for the weighting terms reveals that for this example, the weights for the small zones get large in comparison to the weights for the large zones. The small zones with their large weights, then, have more of an influence on the diffusive mesh relaxer. The algorithm which best retains the original zoning structure is the diffusive mesh relaxer with $r=1$.

The next zoning variation for the simple cube test case uses a high density of zones in 
half the region, as shown in Fig. 32 .

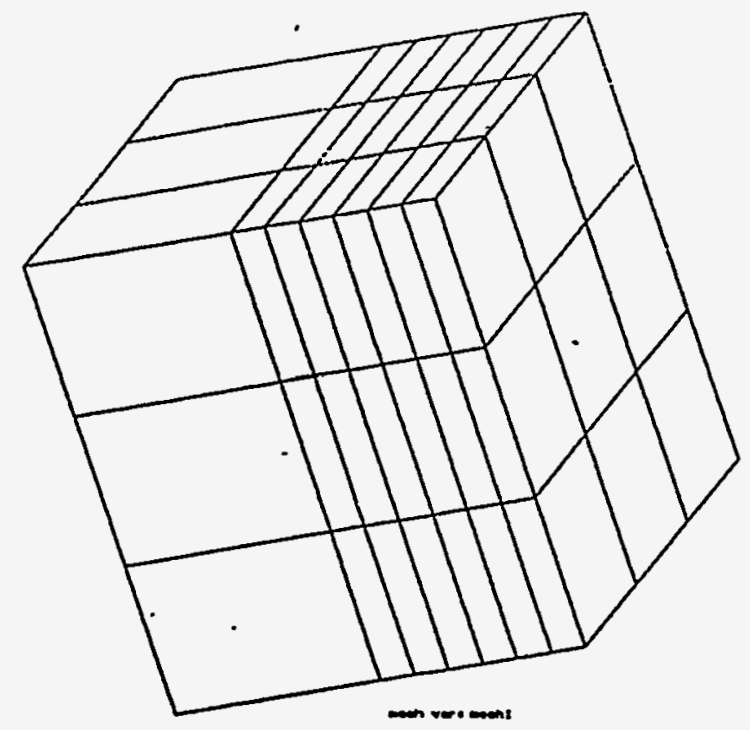

Fig. 32: Original Zoning Structure of Second Test Case.

An orthogonal slice is taken through the cube, and the $2 \mathrm{D}$ plot of this slice is shown in Fig. 33 .

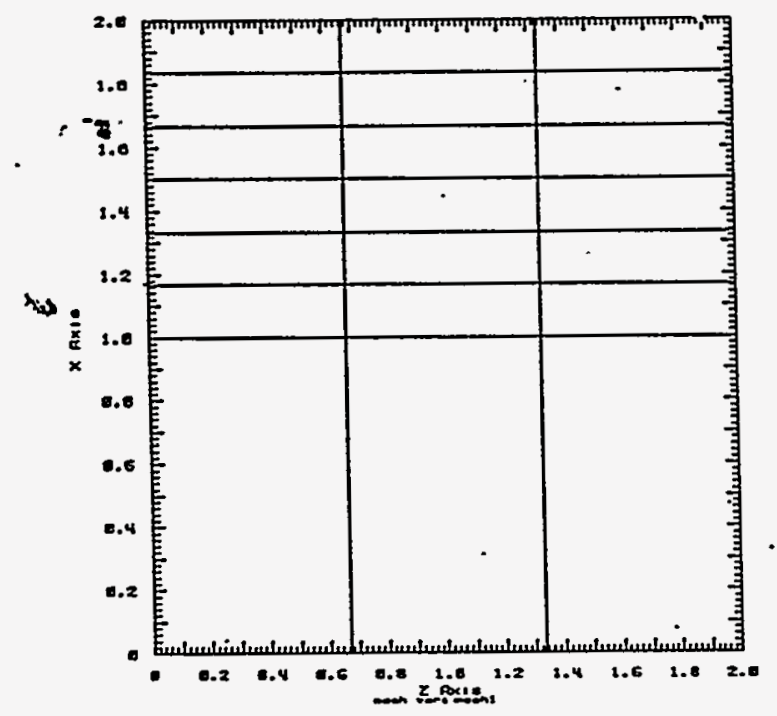

Fig. 33: Orthogonal Slice of Second Test Case 
The resulting mesh for several values of $r$ are shown in Fig. 34 through Fig. 37 .

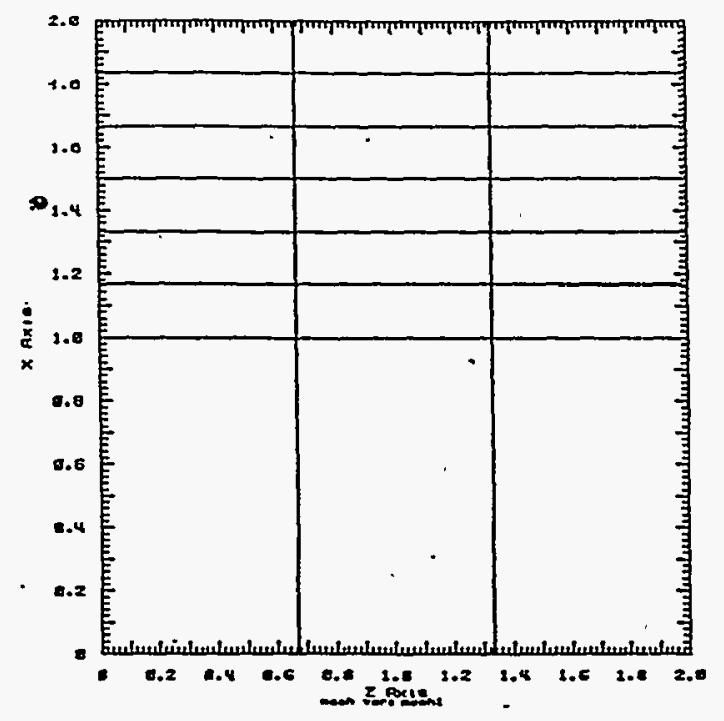

Fig. 34: Orthogonal Slice of Diffusive Mesh Relaxer with $r=1$.

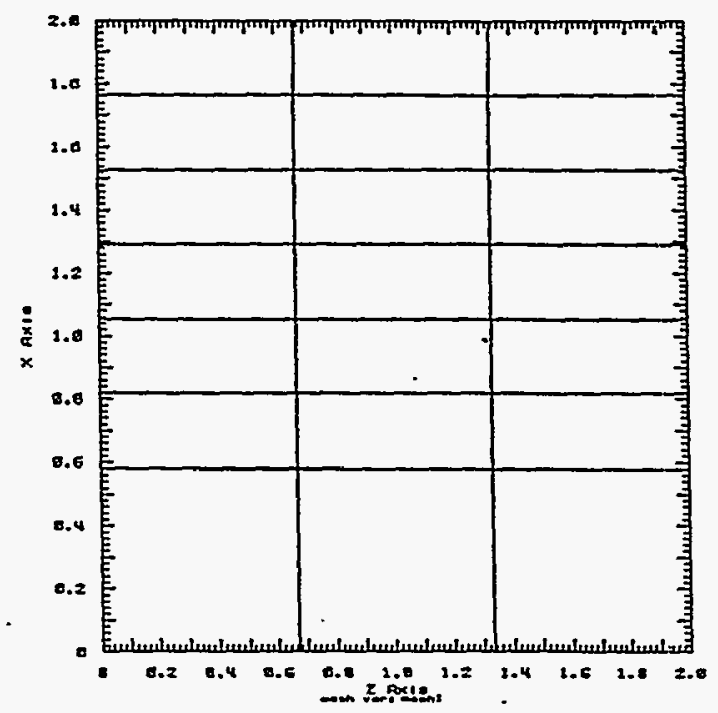

Fig. 35: Orthogonal Slice of Diffusive Mesh Relaxer with $r=1 / 2$. 


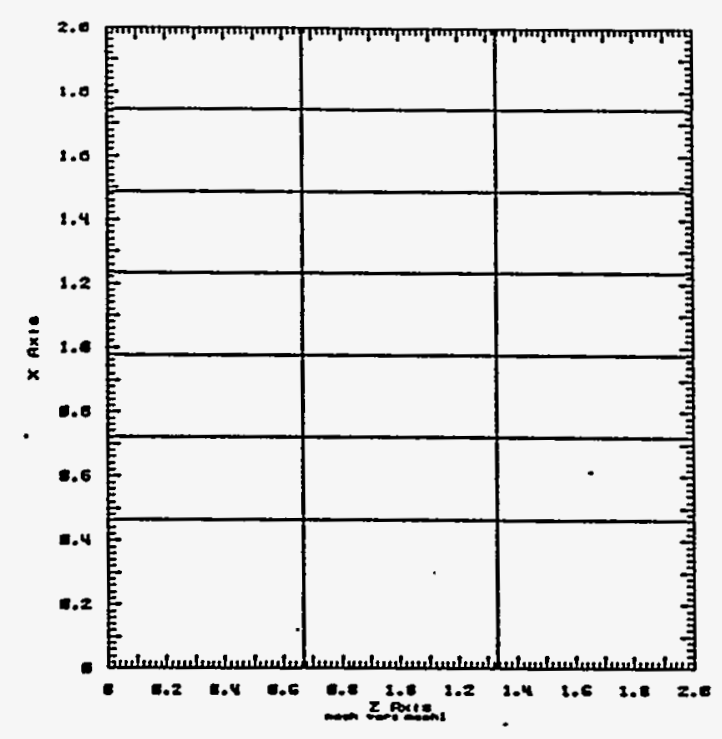

Fig. 36: Orthogonal Slice of Diffusive Mesh Relaxer with $r=1 / 3$.

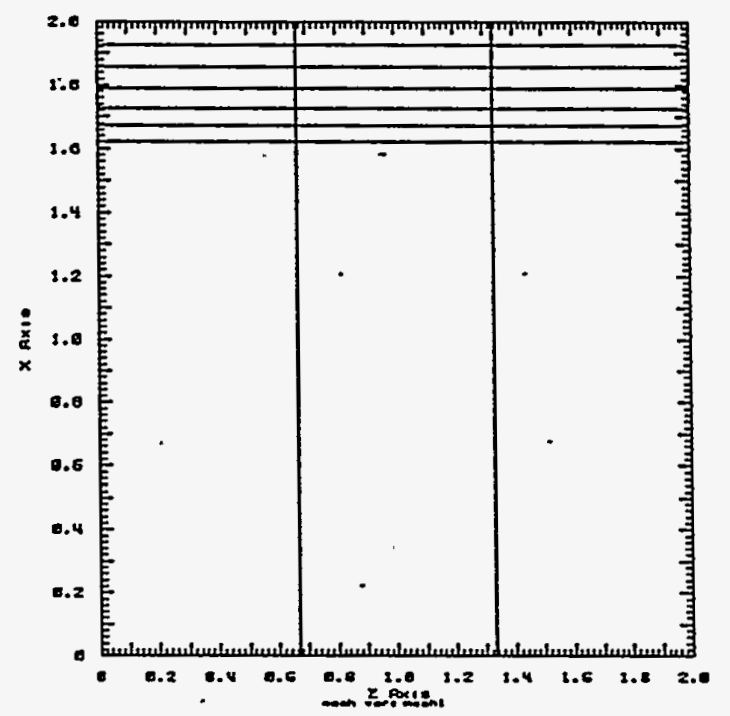

Fig. 37: Orthogonal Slice of Diffusive Mesh Relaxer with $r=2$. 
For comparison, it is instructive to relax the original mesh using the Equipotential method. The resulting mesh is seen in Fig. 38 .

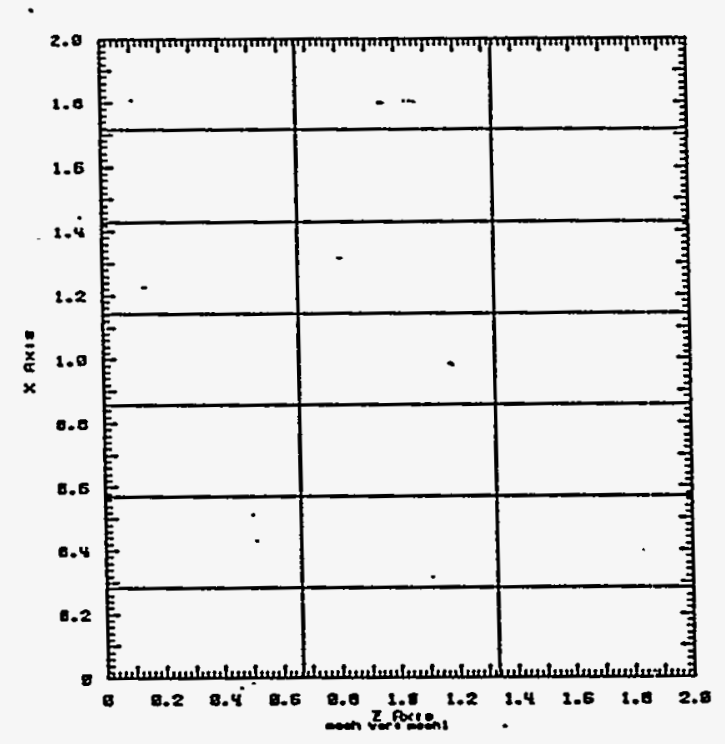

Fig. 38: Orthogonal Slice of Equipotential Mesh Relaxer.

Observations similar to that of the simple cube test case discussed previously can be made about these results. As the value for $r$ decreased, the resulting mesh structure approached that of the Equipotential mesh relaxer. The opposite effect occurs when $r$ increases. The algorithm which best retains the original zoning structure is the diffusive mesh relaxer with $r=1$.

The final zoning variation for the simple cube test case uses ratio zoning. An orthogonal slice is taken through the cube, and the 2D plot of this slice is shown in Fig. 39 . The resulting mesh using the diffusive mesh relaxer with $r=1$ is shown in Fig. $\overline{4} 0$. 


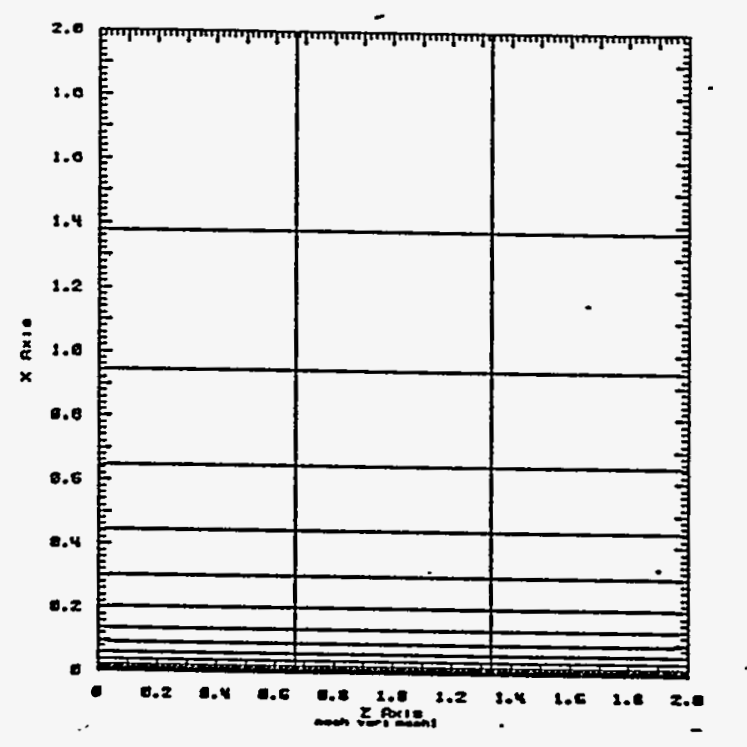

Fig. 39: Orthogonal Slice of Ratio Zoning Test Case

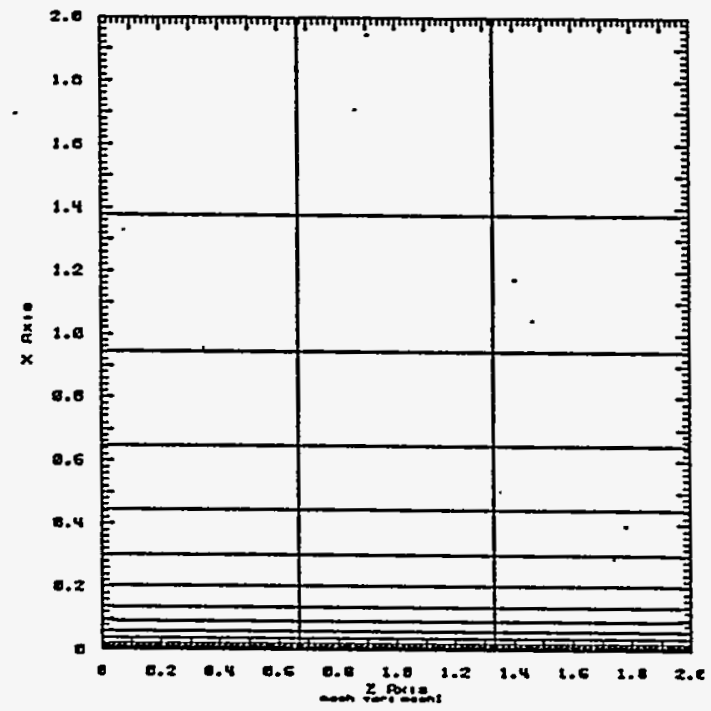

Fig. 40: Orthogonal Slice of Diffusive Mesh Relaxer with $r=1$. 
The resulting mesh using the Winslow-Crowley or Equipotential method is seen in Fig. 41.

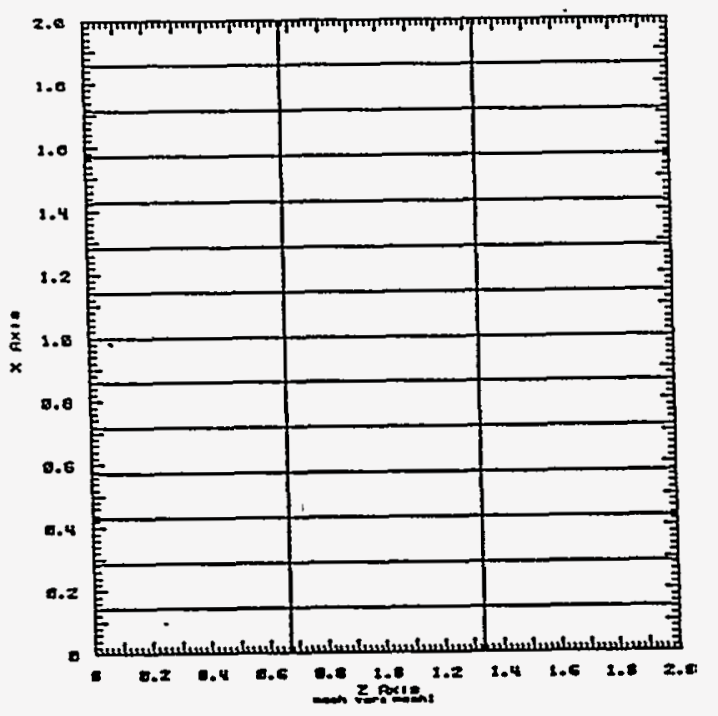

Fig. 41: Orthogonal Slice of Equipotential Mesh Relaxer.

The algorithm which best retains the original ratio zoning structure is the diffusive mesh relaxer with $r=1$.

\section{Test Problem: Spherical Symmetry}

This problem tests the diffusive mesh relaxer for spherical symmetry. An inner region of low pressure gas is surrounded by spherical shells of high pressure gas. A slide surface separates the low pressure gas from the outer shells. The inner region is treated with ALE techniques, and the outer shells are Lagrangian. Due to the symmetry of the problem, a quarter of the actual sphere is modeled, as seen in Fig. 42 . An orthogonal slice is taken, and the 2D plot of this slice is shown in Fig. 43 . Fig. 44 through Fig. 49 illustrate the progress of the problem as the outer shells compress the inner region of low pressure gas. For this test case, $r=1$. 


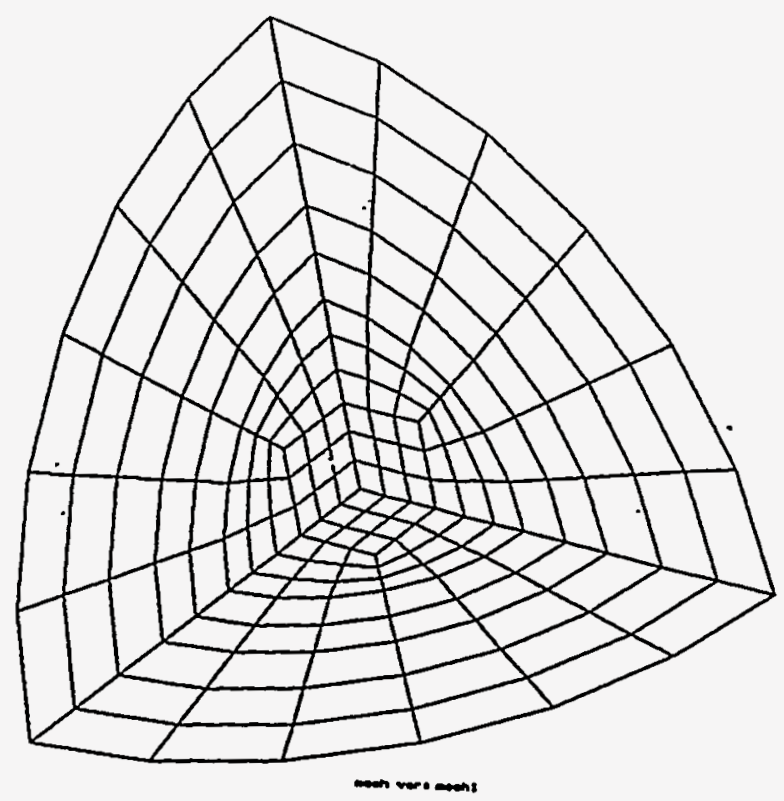

Fig. 42: Original Mesh of the Spherical Symmetry Test Case.

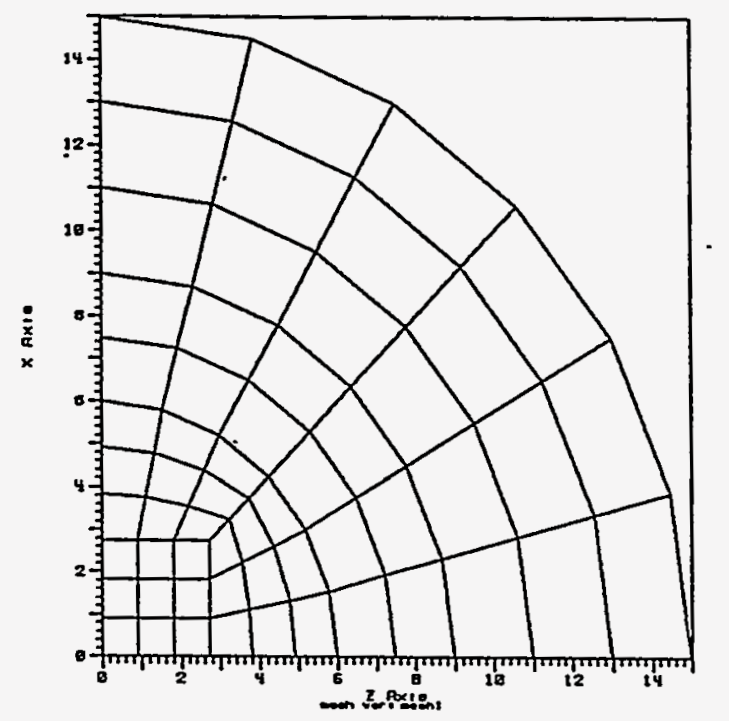

Fig. 43: Orthogonal Slice of the Spherical Symmetry Test Case. 


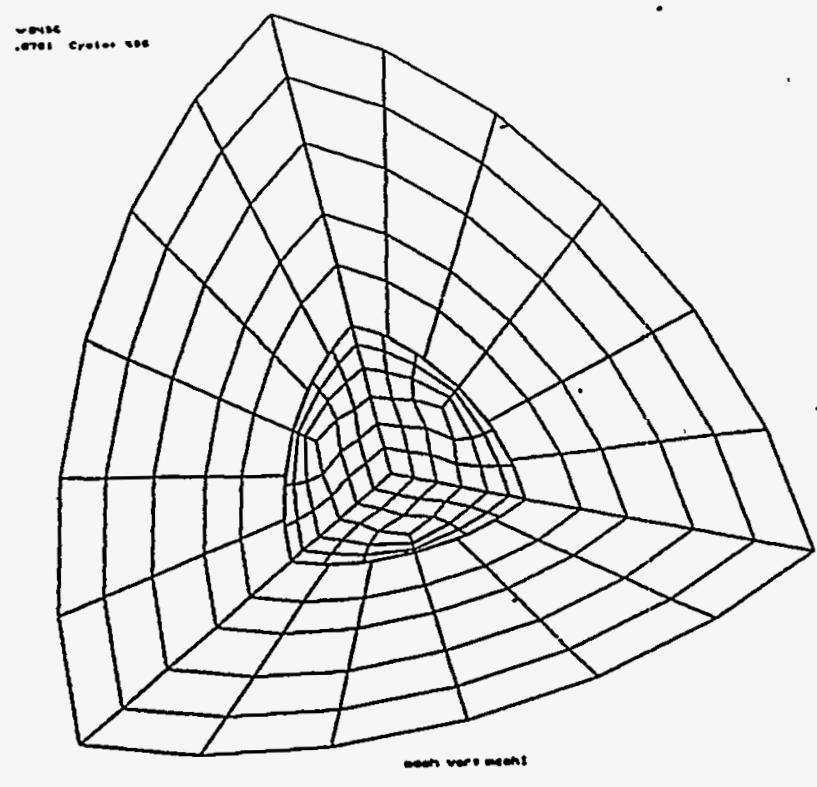

Fig. 44: 3D Mesh of the Spherical Symmetry Test Case at 16us.

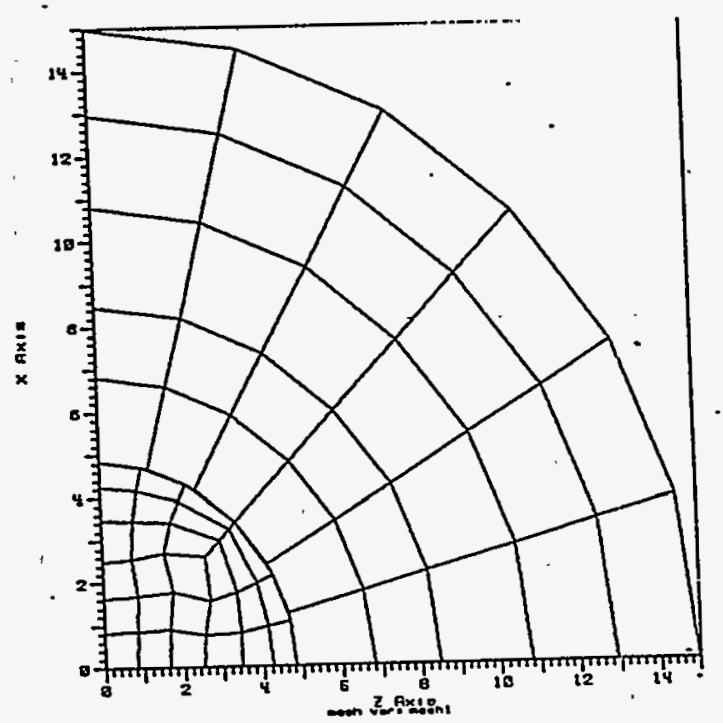

Fig. 45: Orthogonal Slice of the Spherical Symmetry Test Case at $16 \mu$ s. 


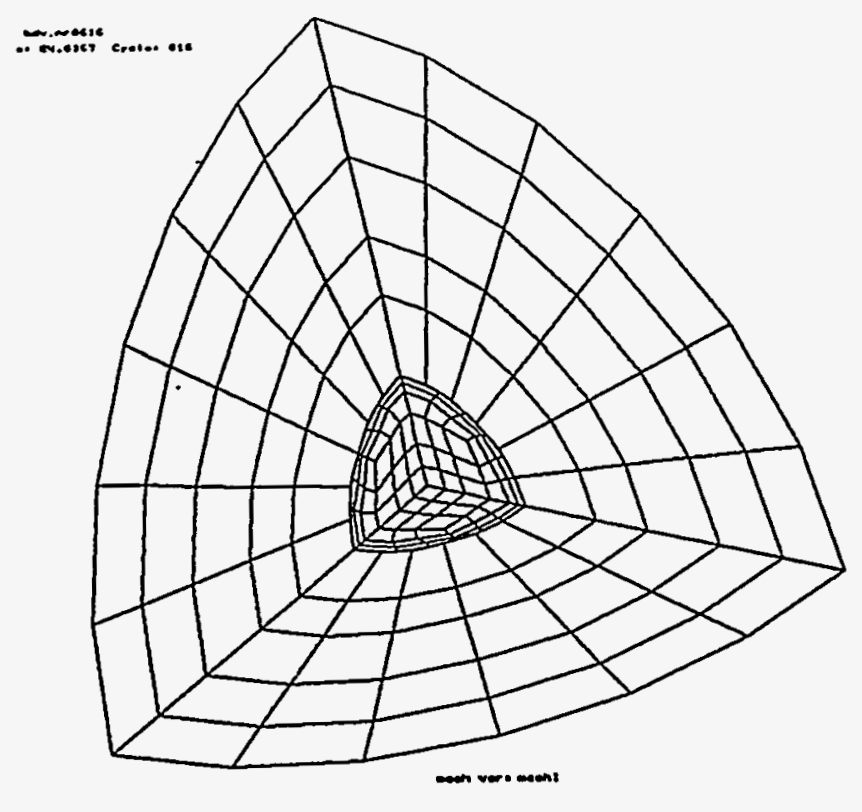

Fig. 46: 3D Mesh of the Spherical Symmetry Test Case at $24 \mu \mathrm{s.}$

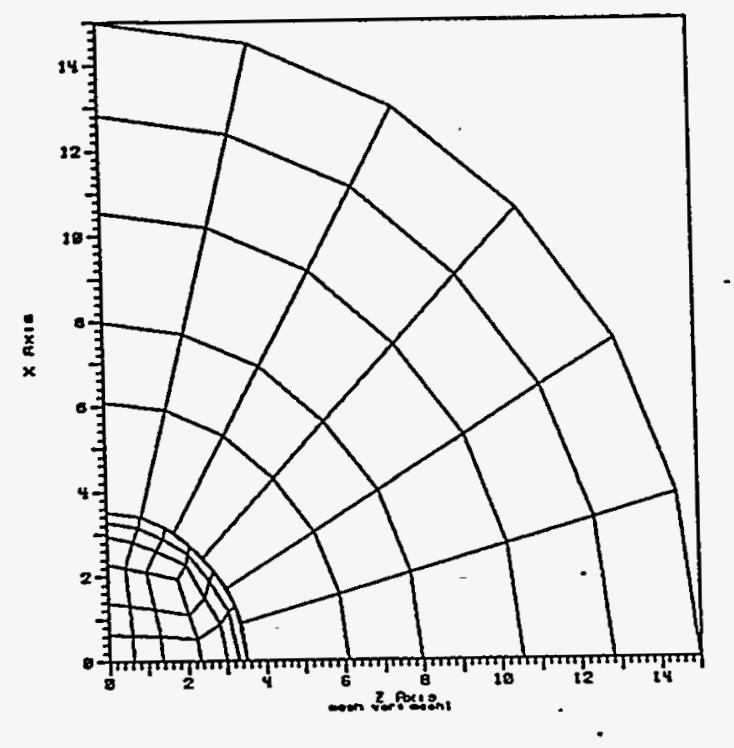

Fig. 47: Orthogonal Slice of the Spherical Symmetry Test Case at $24 \mu \mathrm{s}$. 


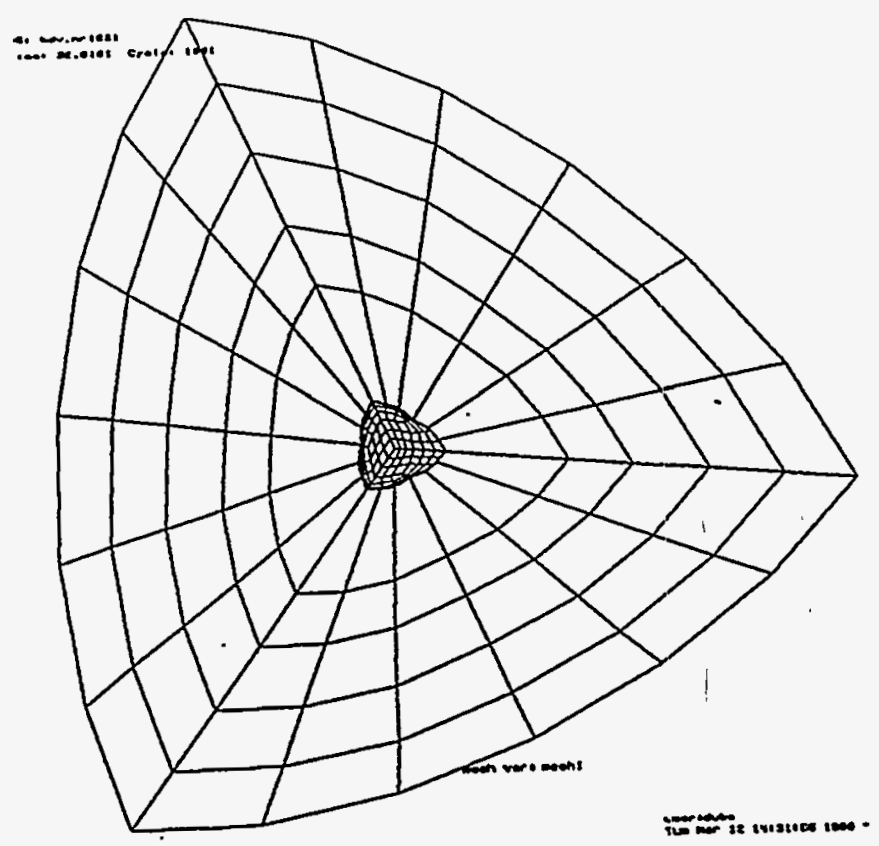

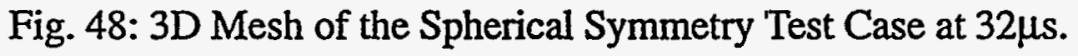

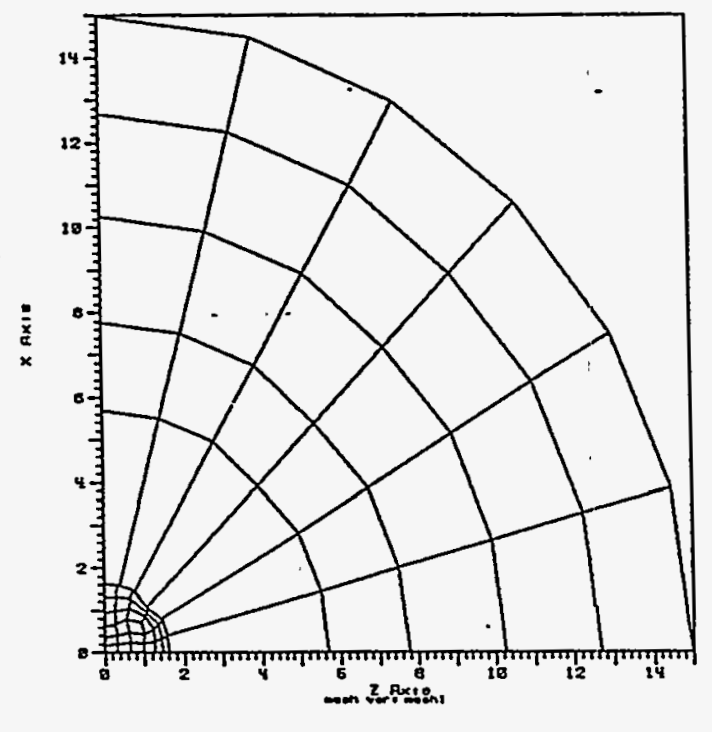

Fig. 49: Orthogonal Slice of the Spherical Symmetry Test Case at $32 \mu s$. 
The purpose of this test case was actually twofold. First, this problem tested spherical symmetry, and the diffusive mesh relaxer behaved appropriately. Second, this case demonstrated the diffusive mesh relaxer's ability to contract the resulting mesh when the outer surface was collapsing, while still retaining the original zoning structure.

\section{Test Problem: Air Flow Through a Diverging Nozzle}

This next problem tests the ability of the diffusive mesh relaxer to handle a fast moving fluid through a diverging nozzle. A $3 D$ image of the problem is shown in Fig. 50, including a view of the zoning for the nozzle and the air. The 2D plot of an orthogonal slice is seen in Fig. 51 . The nozzle is zoned using shell elements, and is treated as Lagrangian, while the fluid, air zoned as brick elements and treated with ALE, is moving at $0.01 \mathrm{~cm} / \mu \mathrm{s}$. Note that a shell element is a two-dimensional element with a given thickness. A slide surface exists between the nozzle and the air. Recall that in a diverging noz- 
zle, the direction of flow is from the small end towards the large end.

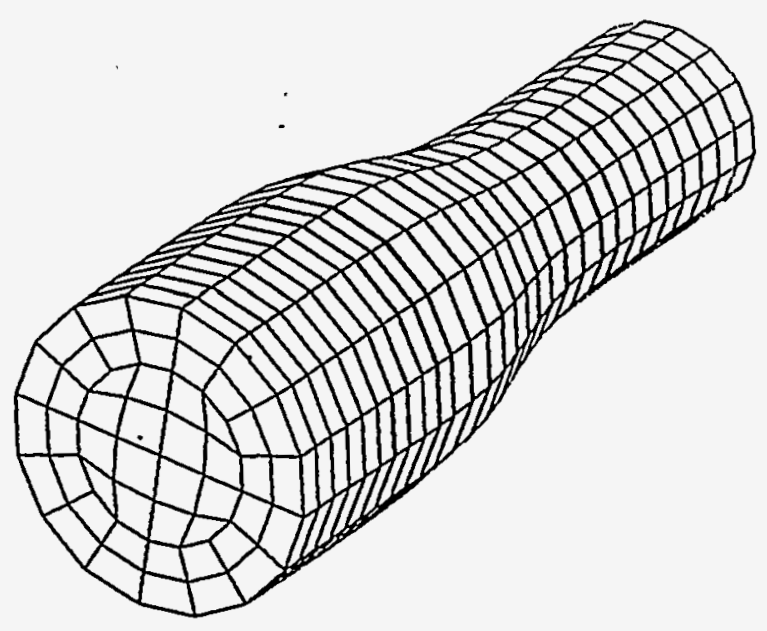

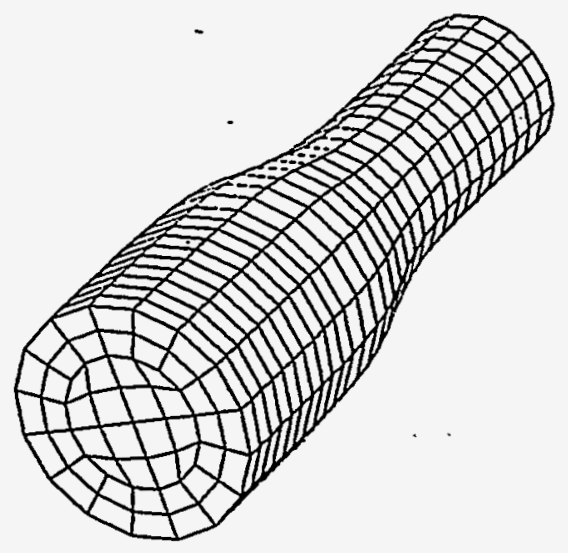

AIR

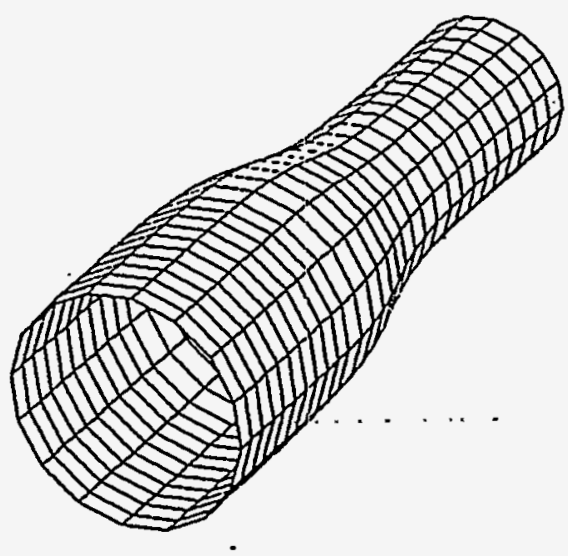

NOZZLE

Fig. 50: 3D Image of Diverging Nozzle Test Case. 


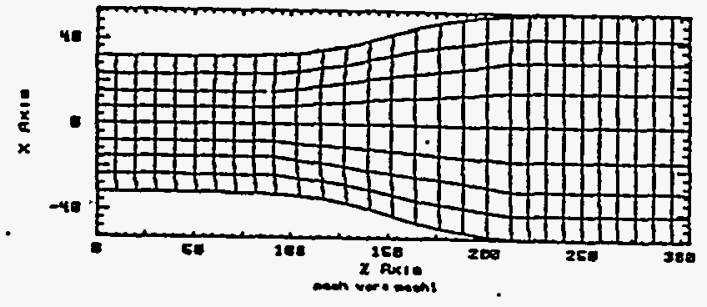

Fig. 51: Orthogonal Slice of Diverging Nozzle Test Case

The Equipotential method causes the zones at the outlet of the nozzle to bunch up, as seen in Fig. 52

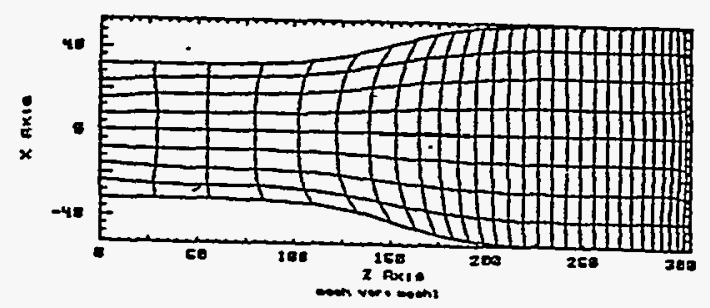

Fig. 52: Orthogonal Slice of Diverging Nozzle Using Equipotential Mesh Relaxer at 005s. 
This method is unable to successfully relax the changing mesh as the fluid moves through the mesh.

The diffusive mesh relaxer, though, is able to relax the mesh in the midst of the fast moving fluid, seen in Fig. 53 , and is reasonably able to maintain the initial zoning structure, except at the slide surface between the nozzle and the air.

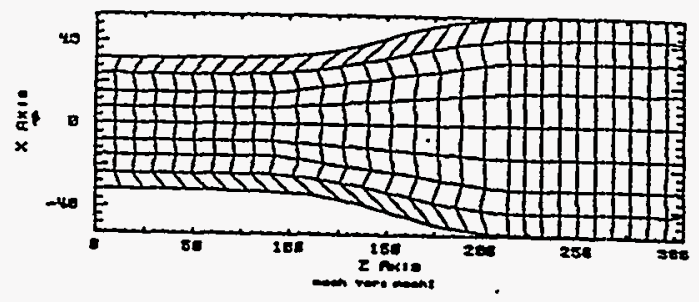

Fig. 53: Orthogonal Slice of Diverging Nozzle Using Diffusive Mesh Relaxer at 036s.

The slide surface used an Equipotential mesh relaxation technique, which was having trouble keeping up with the fluid flow, and thus affected the zones for the boundary elements between the air and nozzle. One area of future work is to apply the diffusive mesh relaxer algorithm to slide surfaces.

\section{Test Problem: Spherical Symmetry With a Push at One Point}

The following problem is similar to the spherical symmetry test case, but gives the mesh a push at one point in the ALE material. The purpose of this push is to test the abil- 
ity of the diffusive mesh relaxer to handle "S" shaped surfaces. The problem consists of an inner region, which is treated by the ALE mode, three outer regions treated in Lagrangian mode, and a slide surface separating the inner region from the outer region. Due to the symmetry of the problem, only a quarter of the problem is modeled, as seen in Fig. 54 . The 2D plot of an orthogonal slice of this initial zoning structure is seen in Fig. 55 . For this particular problem, the initial mesh is first relaxed using many iterations of the Equipotential method to alleviate the stiff zoning structure in the advecting region, and this resulting relaxed mesh is shown in Fig. 56 . The resulting meshes when using the Equipotential relaxer for the duration of the problem are seen in Fig. 57 - Fig. 59 while the results of the diffusive mesh relaxer are seen in Fig. 60 - Fig. 62 .

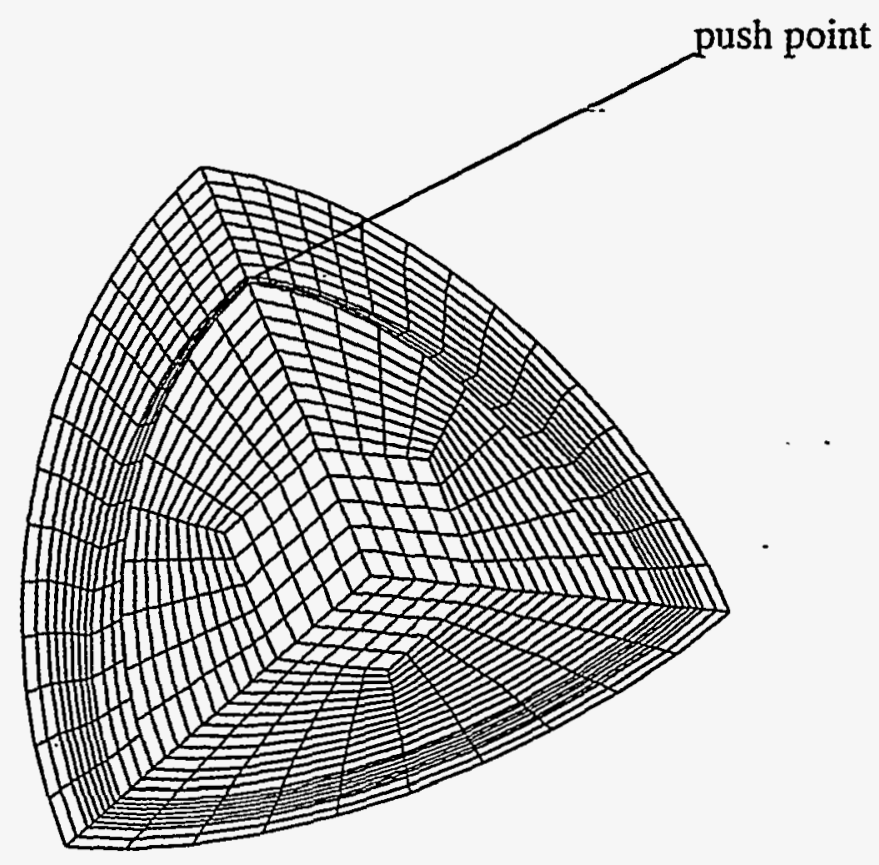

Fig. 54: Original Zoning Structure of Push Test Case. 


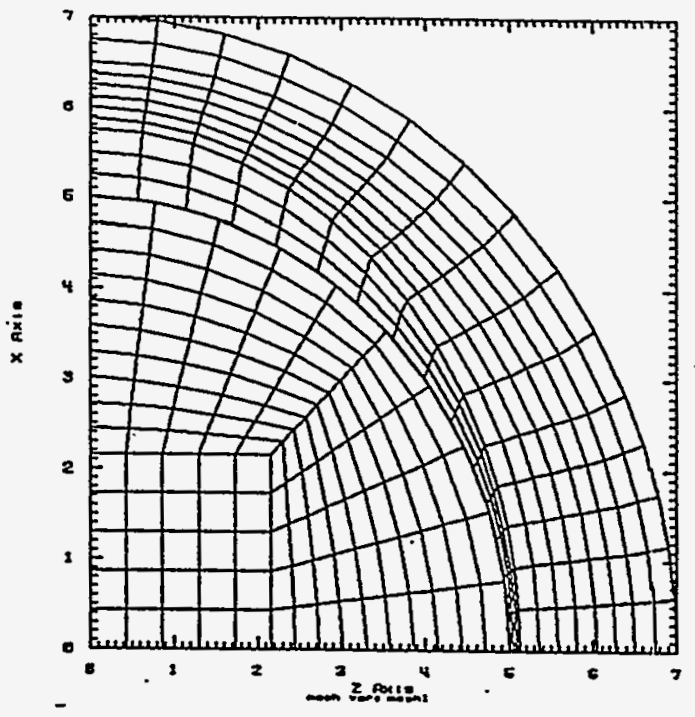

Fig. 55: Orthogonal Slice of the Original Zoning for the Push Test Case.

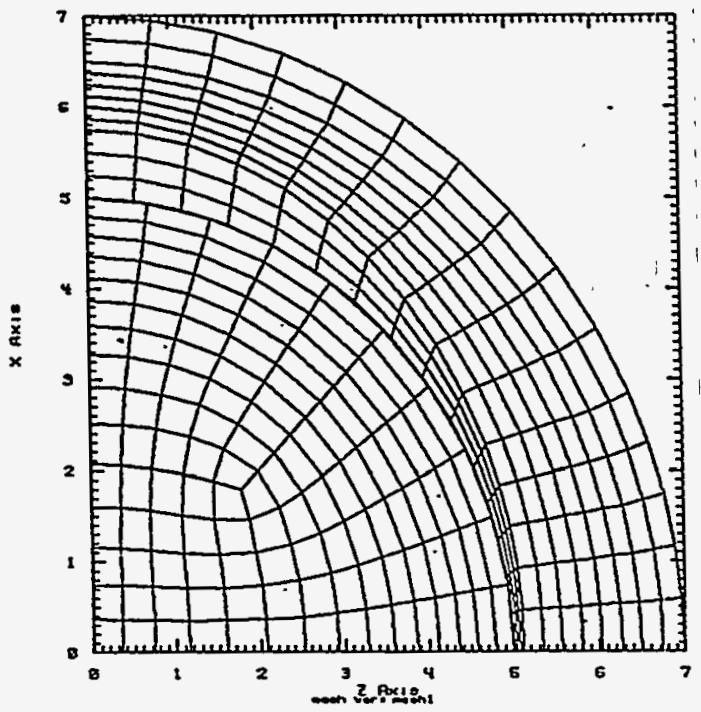

Fig. 56: Orthogonal Slice of Initial Relaxed Mesh for Push Test Case. 


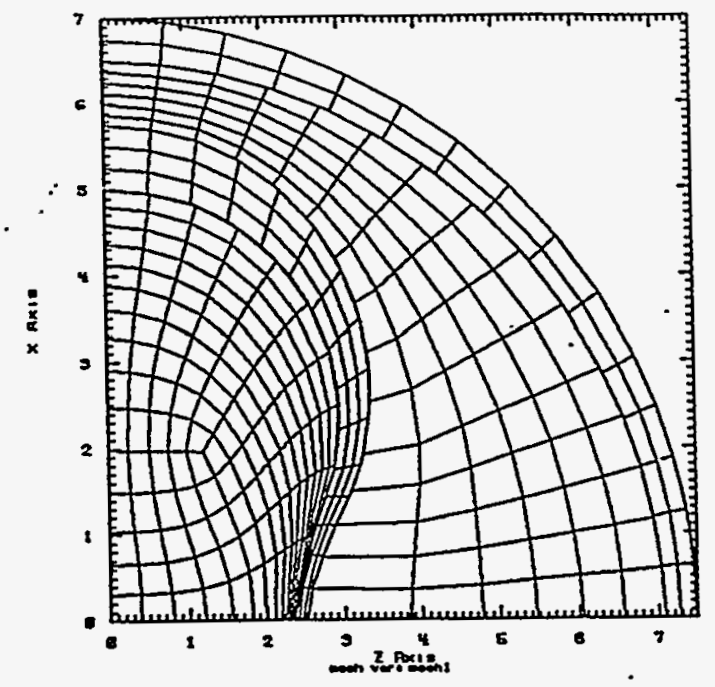

Fig. 57: Orthogonal Slice Using Equipotential Algorithm at 10 $\mu$ s.

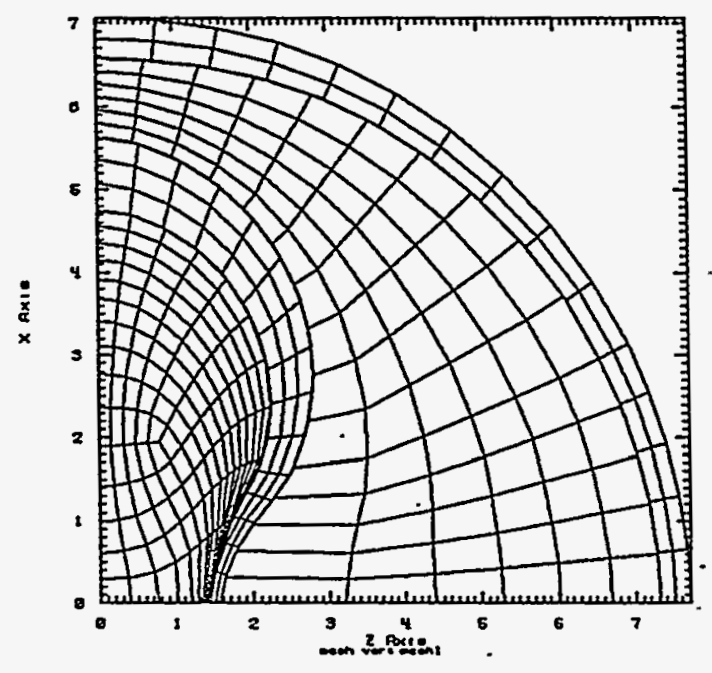

Fig. 58: Orthogonal Slice Using Equipotential Algorithm at $12 \mu \mathrm{s}$. 


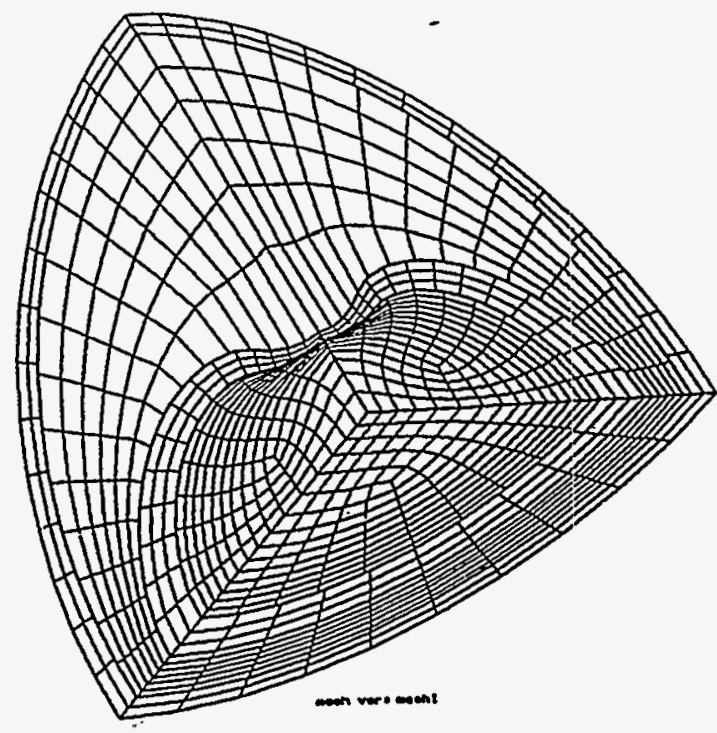

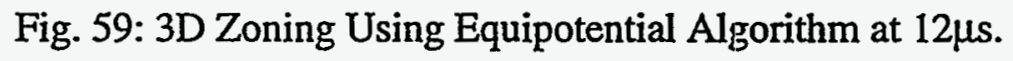

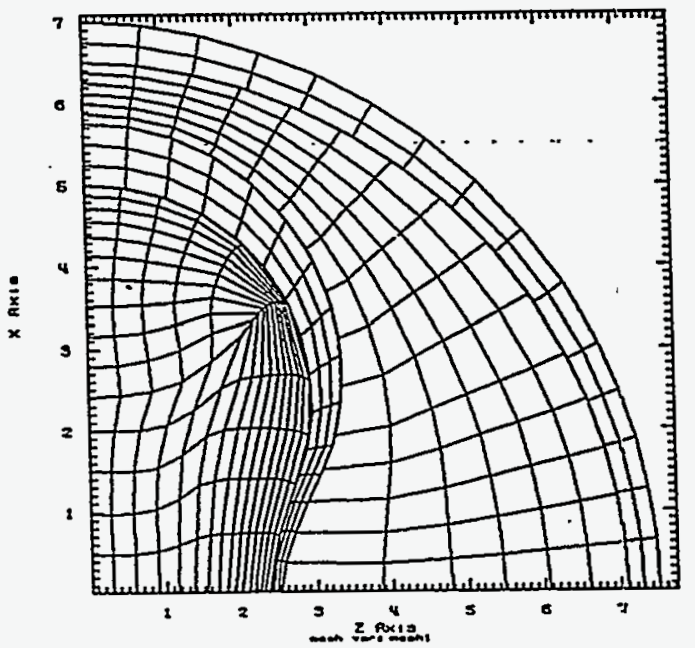

Fig. 60: Orthogonal Slice Using Diffusive Algorithm with $r=I$ at $10 \mu \mathrm{s}$. 


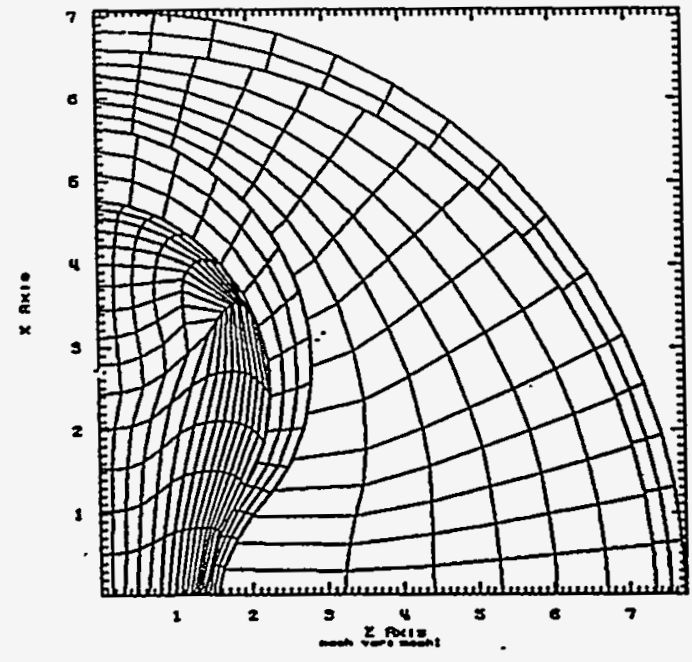

Fig. 61: Orthogonal Slice Using Diffusive Algorithm with $r=1$ at $12 \mu \mathrm{s}$.

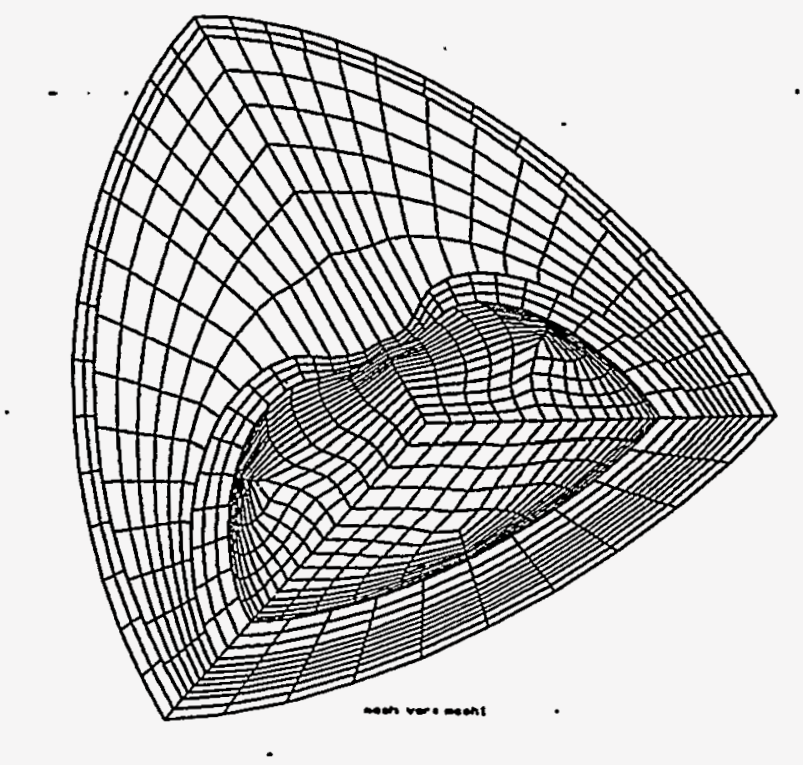

Fig. 62: 3D Zoning Using Diffusive Algorithm with $r=l$ at $12 \mu$ s. 
The Equipotential algorithm has trouble with zones bunching up at the incoming surface. The diffusive mesh relaxer is able to avoid this problem, and maintains the initial zoning structure along the axes. However, an interesting phenomena has occurred with the nodes along the $45^{\circ}$ line in the advecting material. These nodes are being pulled towards the outer surface. Although this is not a bad phenomena, it would be interesting to see if this result can be minimized. From previous test cases, it was found that the effects of the diffusive mesh relaxer can be controlled by varying the power, $r$, on the weighting term. Fig. 60 - Fig. 62 use a weight power of 1. Fig. 63 -Fig. 65 use a weight power of $1 / 3$.

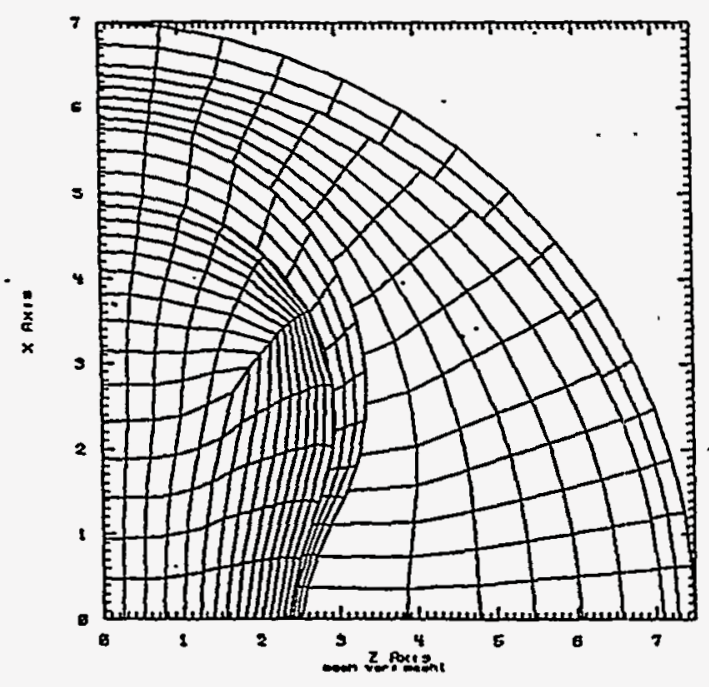

Fig. 63: Orthogonal Slice Using Diffusive Algorithm with $r=1 / 3$ at $10 \mu$ s. 


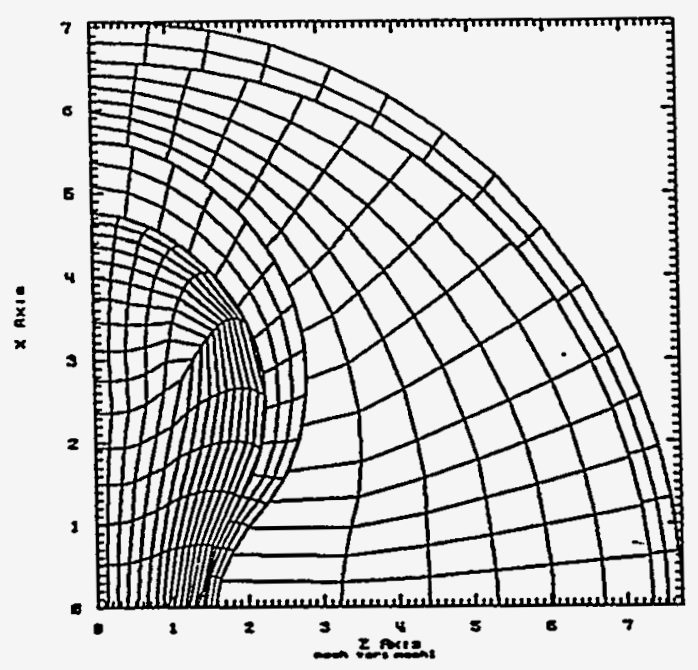

Fig. 64: Orthogonal Slice Using Diffusive Algorithm with $r=1 / 3$ at $12 \mu$ s.

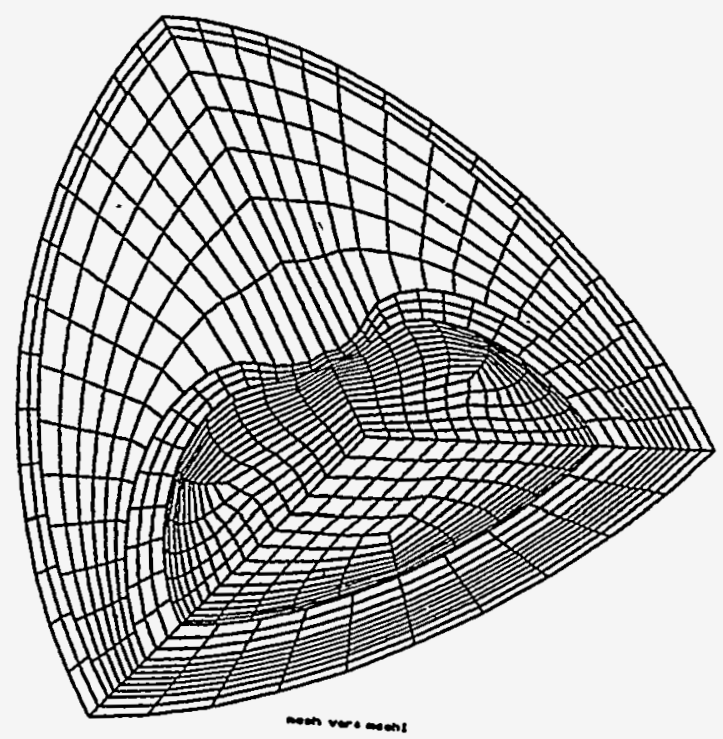

Fig. 65: 3D Zoning Using Diffusive Algorithm with $r=1 / 3$ at $12 \mu$ s. 
When $r=1$, the weights for the elements were of order 1 or 2 . Letting $r=1 / 3$

caused the resulting weights to be close to 1 , and close to each other in magnitude, and thus decreased the affect of the diffusive mesh relaxer.

The problem with the mesh generated via the Equipotential method in Fig. 57 - Fig. 59 is similar to that seen in the diverging nozzle problem. The bunching of zones seen along the horizontal axis is due to the algorithm's inability to relax the changing mesh in the wake of a fast moving fluid. The resulting mesh in Fig. 63 - Fig. 65 , using the diffusive mesh relaxer with $r=1 / 3$, produced a zone structure that was able to relax this "S" shaped curve.

\section{Test Problem: Aerostructural Test Case}

A major motivating factor for developing the diffusive mesh relaxer was the need to have a relaxer maintain the existing zone structure of a problem. The Equipotential algorithm, by design, tries to create equal spaced zones. In some problems, however, this resulting mesh is inadequate for properly simulating a Continuum Mechanics problem. The following test case demonstrates this serious failing of the Equipotential method. The aerostructural test case consists of a nacelle, center-body and wing section for an engine in a wind tunnel associated with the High Speed Civil Transport Program. The nacelle, center-body and wing section are represented by shell elements and treated in Lagrangian mode, while brick elements are mapped on to the structure to treat the air flow, moving at Mach 2.1, in advecting mode. The computational geometry for this test 
case is shown in Fig. 66 .

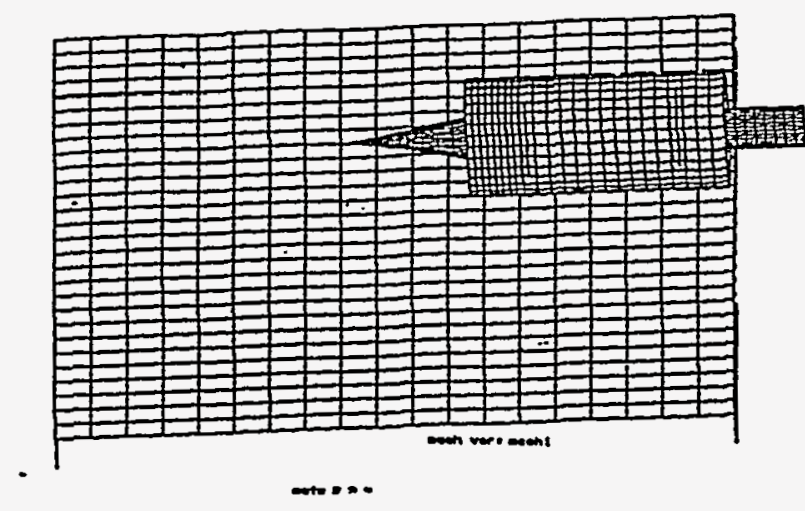

Fig. 66: Original Zoning for Aerostructural Test Case.

The area of interest in this calculation is the air flow around the center-body, and the $2 D$ plot of an orthogonal slice of the initial zoning structure is shown in Fig. 67 . During the calculation, this initial zoning structure is desired, especially the fine zoning around the center-body since it is critical for properly modeling the behavior of the air in this region. If the Equipotential algorithm is used, these areas of fine zoning are lost, and the mesh relaxes to that shown in Fig. 68 . The diffusive mesh relaxer, however, is able to 
maintain the initial zoning structure, as seen in Fig. 69 .

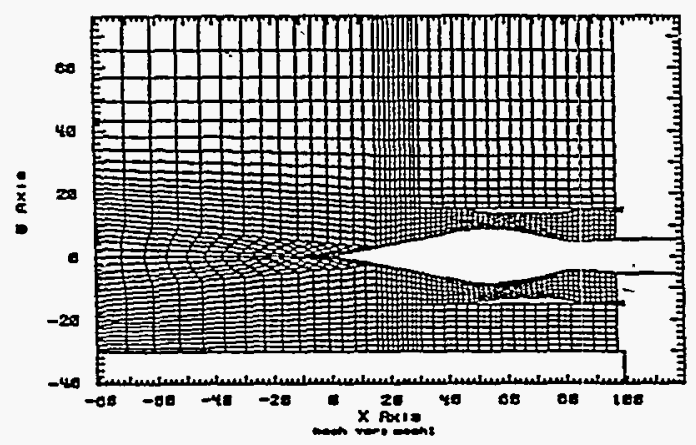

Fig. 67: Orthogonal Slice of Initial Zoning for Aerostructural Test Case.

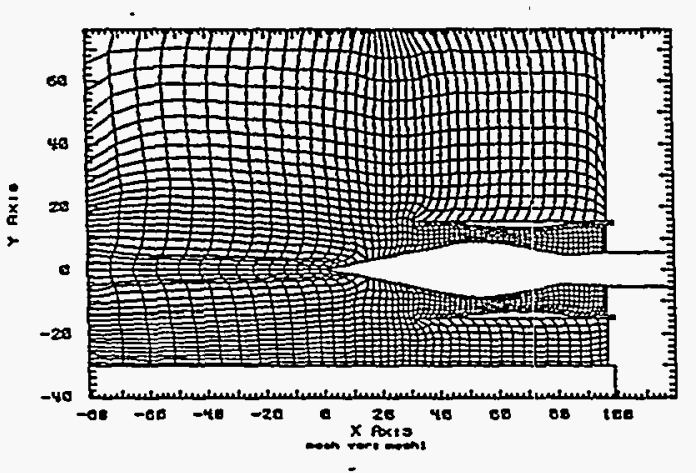

Fig. 68: Orthogonal Slice using Equipotential Method for Aerostructural Test Case at $33 \mu \mathrm{s}$. 


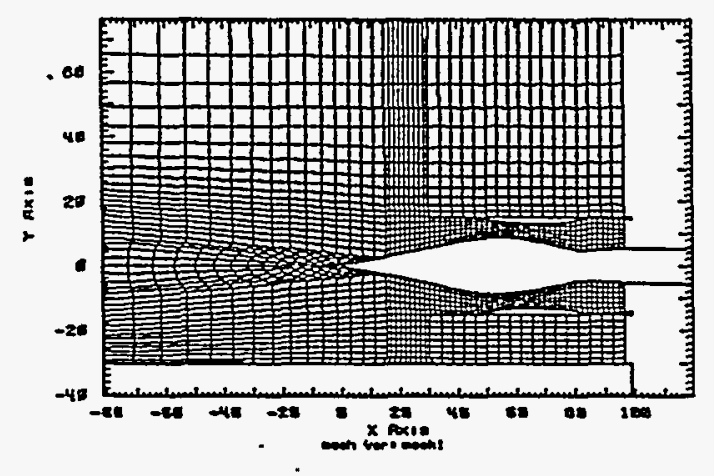

Fig. 69: Orthogonal Slice using Diffusive Method for Aerostructural

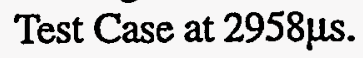




\section{Chapter 7: Conclusions}

Simulating Continuum Mechanics problems with the use of ALE techniques has been developing over the past 15 years, and the use of this technique is gaining visibility by proving to be a successful approach to solving this class of problems. As the technique has expanded, so has the need for better controls on the relaxation phase of the ALE step. This dissertation presents a useful mesh relaxation method.

The theory and application for a diffusive mesh relaxation technique has been developed in the previous chapters. Starting with an overview of mesh generation was a natural lead into the development of the diffusive mesh relaxation technique. After initial experiments with the diffusive relaxer showed hourglass problems which lead to instabilities in the resulting mesh, the numerical solution was modified to control these unphysical modes in the mesh. A brief introduction to Lagrangian mechanics was given, and then the application to five different test problems was discussed.

The five test problems in Chapter 6 have shown that the diffusive mesh relaxer produces smooth, regular meshes, and is able to maintain an existing zone structure during the mesh relaxing phase of an ALE calculation. In two cases, the air flow through a diverging nozzle and the aerostructural problem, the diffusive mesh relaxer was a necessity for the problem to run. In another case, the spherical symmetry problem with a push at one point, the resulting mesh from the diffusive mesh relaxer was better than that using the Equipotential algorithm.

The goal of this dissertation was to develop an unstructured, hexahedral mesh relaxation approach for use in the first phase of the advection step in an ALE calculation. To be successful, this method needs to produce smooth, regular meshes, with the additional 
constraint that it maintain the existing zone structure of the initial mesh. The diffusive mesh relaxation algorithm achieves this goal. 


\section{Chapter 8: $\quad$ References}

1. A. A. Amsden and C. W. Hirt, A Simple Scheme for Generating General Curvilinear Grids, J. Comput. Phys., Academic Press, 11, 1973, pp 348-359.

2. G. Arfken, Mathematical Methods for Physicists, Third Edition, Academic Press, Inc., Orlando, 1985, pp. 925-928.

3. J.U. Brackbill and J.S. Saltzman, Adaptive Zoning for Singular Problems in Two Dimensions, J. Comput. Phys. 46, 1982, pp. 342-368.

4. H. S. Carslaw and J. C. Jaeger, Conduction of Heat in Solids, Second Edition, Oxford University Press, New York, 1959, pp. 1-6.

5. J. E. Castillo, ed., Mathematical Aspects of Numerical Grid Generation, SIAM Frontiers in Applied Mathematics, Philadelphia, 1991, pp. xiii-2.

6. R. Cook, D. S. Malkus, M. E. Plesha, Concepts and Applications of Finite Element Analysis, Third Edition, John Wiley, New York, 1989, pp. 190-194, pp. 461-468, pp. 563-564.

7. R. Couch, E. Albright, and N. Alexander, JOY Computer Code, Lawrence L:ivermore National Laboratory, Livermore, CA, UCD-19688, 1983.

8. R. Couch, R. Sharp, I. Otero, R. Tipton, and R. McCallen, Applications of ALE Techniques to Metal Forming Simulations, AMD-Vol 180, Advanced Computational Methode for Material Modeling, American Society of Mechanical Engineers, Book No. H00883, 1993, pg 133-140.

9. W. P. Crowley, An Equipotential Zoner on a Quadrilateral Mesh, Lawrence Livermore National Laboratory, 1962 (unpublished).

10. D. A. Field, Laplacian Smoothing and Delaunay Triangulations, Commun. in Appl. Numer. Meth., John Wiley, New York, 1988, 4, pp. 709-712.

11. D. P. Flanagan and T. Belytschko, A Uniform Strain Hexahedron and Quadrilateral with Orthogonal Hourglass Control, Int. J. Numer. Meths. in Eng., John Wiley, New York, 17, 1981, pp. 679-706.

12. W. H. Frey and D. A. Field, Mesh Relaxation: A New Technique for Improving Triangulations., Int. J. Numer. Meths. in Eng., John Wiley, New York, 31, 1991, pp. 1121-1133.

13. Y. C. Fung, Foundations of Solid Mechanics, Prentice-Hall, Inc., 1965, pp. 61-62, pp. 122-123.

14. G. Golub and C. F. Van Loan, Matrix Computations, Second Edition, John Hopkins University Press, Baltimore, 1989, p. 394, pp. 506 - 509.

15. J. P. Holman, Heat Transfer, Fifth Edition, McGraw-Hill, New York, 1981, pp. 1-7, pp 24-25.

16. A. C. Holt and S. Lake, Joint DoD/DOE Munitions Technology Development Pro- 
gram FY95 Progress Report, Lawrence Livermore National Laboratory UCRL103482-95, 1996, pp. 275-277.

17. D. Kershaw, M. Prasad, and M. Shaw, $3 D$ Unstructured Mesh ALE Hydrodynamics with Upwind Discontinuous Finite Element Method, Lawrence L:ivermore National Laboratory, Livermore, CA, UCRL-xxxxx, 1996.

18. P. Knupp, A Robust Elliptic Grid Generator, J. Comput. Phys., Academic Press, 100, 1992, pp. 409-418.

19. P. Knupp and S. Steinberg, Fundamentals of Grid Generation, CRC Press, Inc., 1993, pp. 1-9, pp. 103-108, pp. 119-122.

20. D. Kosloff and G. A. Frazier, Treatment of Hourglass Patterns in Low Order Finite Element Codes, Int. J of Numer. and Analy. Meths Geomechanics, John Wiley, 2, 1978, pp. 57-72.

21. L. Margolin and A. E. Tarwater, Diffusion Operator on Lagrangian Meshes, Lawrence Livermore National Laboratory, Livermore CA, UCRL-95652, 1986.

22. G. Mase, Continuum Mechanics, Schaum's Outline Series in Engineering, McGrawHill, New York, 1970, pp. 79-81, pp. 110-116, pp. 126-127.

23. C. W. Mastin and J.F. Thompson, Elliptic Systems and Numerical Transformations, J. Mathem. Analysis and Apps. 62, 1978, pp 52-62.

24. G. A. Mohr, Finite Elements for Solids, Fluids, and Optimization, Oxford University Press, New York, 1992, pp. 4-6, pp 34-35.

25. R. Rainsberger, D. W. Stillman, J. O. Hallquist, M. A. Christon, and D. Dovey, INGRID User's Maniual, Lawrence Livermore National Laboratory, Livermore CA, UCRL-MA-109790, draft, 1992.

26. R. Sharp, S. Anderson, E. Dube, and I. Otero, Users Manual for ALE3D, Lawrence Livermore National Laboratory, 1996, unpublished.

27. J. Smoller, Shock Waves and Reaction-Diffusion Equations, Springer-Verlag, New York, 1983, pp 64-66.

28. S. Steinberg and P. Roache, Variational Grid Generation, Numer. Methods Partial Diff. Eqs., John Wiley, 2, 1986, pp 71-96.

29. S. Steinberg and P. Roache, Anomalies in Grid Generation on Curves, J. Comput. Phys., Academic Press, 91, 1990, pp 255-277.

30. J. Stoer and R. Bulirsch, Introduction to Numerical Analysis, Springer-Verlag, New York, 1980, p120, p. 432.

31. P. D. Thomas, Construction of Composite Three Dimensional Grids from Subregions Generated by Elliptic Systems, AIAA Computational Fluid Dynamics Conference, Palo Alto, 24, 1981.

32. J. F. Thompson, A Composite Grid Generation Code for General 3D Regions - the EAGLE Code, AIAA Journal, 26, No. 3, AIAA-87-0275, 1988, p. 271. 
33. J.F. Thompson, F. Thames, and C. Mastin, Automatic Numerical Generation of Body-Fitted Curvilinear Coordinate System for Field Containing Any Number of Arbitrary Two-Dimensional Bodies, J. Comput. Phys., Academic Press, 15, 1974, pp 299-319.

34. J.F. Thompson, Z.U.A. Warsi, C.W. Mastin, Numerical Grid Generation: Foundations and Applications, North-Holland, 1985, pp 189-194, p 272.

35. J.F. Thompson, ed., Numerical Grid Generation, North-Holland, 1982, p 137, p169.

36. R. E. Tipton, CALE User's Manual, Lawrence Livernore National Laboratory, Livermore CA, 1990, (unpublished).

37. R. E. Tipton, Grid Optimization by Equipotential Relaxation, Lawrence Livermore National Laboratory, Livermore, CA, 1992, (unpublished).

38. A.M. Winslow, "Equipotential" Zoning of Two-Dimensional Meshes, Lawrence Livermore National Laboratory UCRL-7312, 1963.

39. A.M. Winslow, Numerical Solution of the Quasilinear Poisson Equation in a Nonuniform Triangle Mesh, J. Comput. Phys., Academic Press, 2, 1967, pp 149-172.

40. E. L. Wilson, R. L. Taylor, W. P. Doherty, J. Ghaboussi, Incompatible displacement models, in Numer. and Computer Meths. in Struct. Mechs., Ed. S. J. Fenves et al., Academic Press, New York, 1973, p. 43.

41. B. Van Leer, Towards the Ultimate Conservative Difference Scheme. IV. A New Approach to Numerical Convection, J. Comput. Phys., Academic Press, 23, 1977, pp. 276-299. 